\title{
Homology computations for complex braid groups
}

Received November 10, 2010 and in revised form October 24, 2011

\begin{abstract}
Complex braid groups are the natural generalizations of braid groups associated to arbitrary (finite) complex reflection groups. We investigate several methods for computing the homology of these groups. In particular, we get the Poincaré polynomial with coefficients in a finite field for one large series of such groups, and compute the second integral cohomology group for all of them. As a consequence we get non-isomorphism results for these groups.
\end{abstract}

\section{Contents}

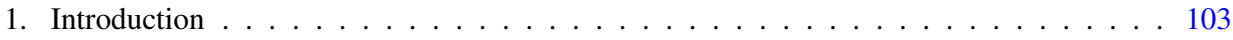

2. Isomorphism and non-isomorphism results for $\mathrm{B}(2 e, e, r) \ldots \ldots \ldots \ldots$

3. Homology of the classical braid group . . . . . . . . . . . . . . . . 111

4. Homology of $\mathrm{B}(2 e, e, r) \ldots \ldots \ldots \ldots \ldots \ldots \ldots$

5. Complexes from Garside theory . . . . . . . . . . . . . . . . . 142

6. The groups $\mathrm{B}(e, e, r) \ldots \ldots \ldots \ldots \ldots \ldots \ldots \ldots$

7. Low-dimensional homology . . . . . . . . . . . . . . . . 155

References . . . . . . . . . . . . . . . . . . . . . . . . . 162

\section{Introduction}

\subsection{Presentation}

The aim of this paper is to provide homological results and tools for the generalized braid groups associated to complex (pseudo-)reflection groups. Recall that a complex reflection group $W$ is a finite subgroup of some $\mathrm{GL}_{r}(\mathbb{C})$ generated by (pseudo-)reflections, i.e. finite-order endomorphisms of $\mathrm{GL}_{r}(\mathbb{C})$ which leave invariant some hyperplane in $\mathbb{C}^{r}$. The collection $\mathcal{A}$ of hyperplanes associated to reflections of $W$ is a central hyperplane arrangement in $\mathbb{C}^{r}$. We let $X=\mathbb{C}^{r} \backslash \cup \mathcal{A}$ denote the corresponding hyperplane complement. The generalised braid group $B=\pi_{1}(X / W)$ is an extension of $W$ by $P=\pi_{1}(X)$. When $W$ is a finite Coxeter group, $B$ is an Artin group of finite Coxeter type.

F. Callegaro: Scuola Normale Superiore, Piazza dei Cavalieri, 7, 56126 Pisa, Italy; e-mail: f.callegaro@sns.it

I. Marin: Institut de Mathématiques de Jussieu, Université Paris 7,

175 rue du Chevaleret, 75013 Paris, France; e-mail: marin@ @ath.jussieu.fr

Mathematics Subject Classification (2010): 20J06, 20F36, 20F55 
Every $W$ can be decomposed as a direct product of so-called irreducible groups (meaning that their natural linear action is irreducible), and $B$ decomposes accordingly. For homological purposes, by the Künneth formula we can thus assume that $W$ is irreducible.

The irreducible complex reflection groups have been classified in 1954 by Shephard and Todd [ST54]: there is an infinite series $G(d e, e, r)$ with three integer parameters, and 34 exceptions, labelled $G_{4}, \ldots, G_{37}$. Their braided counterparts however are far less understood. It is for instance an open problem to decide the lack of injectivity of $W \mapsto B$. Indeed, two non-isomorphic reflection groups $W_{1}$ and $W_{2}$ can provide isomorphic braid groups $B_{1} \simeq B_{2}$, to the extent that any possible braid group $B$ arises from a 2-reflection group, that is, a complex reflection group $W$ whose reflections have order 2.

Recall that $X$ and $X / W$ are $K(\pi, 1)$-spaces by work of [FN62, Bri73, Del72, Ban76, Nak83, OT92, Bes07]. From this, by general arguments, one can however prove that both the rank $r$ of $W$ and the number $|\mathcal{A} / W|$ of $W$-orbits in $\mathcal{A}$ is detected by $B$ :

Proposition 1.1. The homological dimension of $B$ is equal to the rank of $W$. Moreover $H_{1}(B, \mathbb{Z})$ is a free module of dimension $|\mathcal{A} / W|$.

Proof. It is known that $X / W$ is an affine variety of (complex) dimension $r$, homotopically equivalent to a finite $\mathrm{CW}$-complex of dimension $r$. Moreover, the $r$-th cohomology group with trivial coefficients of $P=\operatorname{Ker}(B \rightarrow W)$ is non-zero. Indeed, the Poincaré polynomial of $X$ is $\left(1+c_{1} t\right) \ldots\left(1+c_{r}\right) t$ (see [OT92, Cor. 6.62]) where the $c_{i}$ are positive numbers, called the co-exponents of $W$. In particular, $H^{r}(P, \mathbb{Q})=H^{r}(X, \mathbb{Q}) \neq 0$, and since $P<B, B$ has homological dimension at least $r$, hence exactly $r$, which proves the first part. The second part is proved in [BMR98].

As opposed to the case of Artin groups of finite Coxeter type, for which there are uniform 'simplicial' theories and homological methods, it seems that different methods have to be used in order to deal with these complex braid groups in general. Due to some of the coincidences mentioned above, the groups $B$ provided by the 3-parameter series $G(d e, e, r)$ actually arise from two a priori disjoint series with two parameters, $G(2 e, e, r)$ and $G(e, e, r)$, of 2-reflection groups. The corresponding braid groups $\mathrm{B}(2 e, e, r)=\mathrm{B}(d e, e, r)$ for $d>1$ and $\mathrm{B}(e, e, r)$ seem to belong to distinct worlds. The first ones can be better understood as subgroups of the usual braid groups, or semidirect products of $\mathbb{Z}$ with an Artin group of affine type, whereas the second ones might be better understood as the groups of fractions of suitable monoids with similar (Garside) properties to the usual braid group; it should be noted for instance that the groups $G(e, e, r)$ are generated by $r$ reflections, hence belong to the class of well-generated groups, for which there is a uniform generalization of the Garside approach (see [Bes07]). Moreover, using a specific Garside monoid recently introduced by Corran and Picantin [CP] to deal with the groups $\mathrm{B}(e, e, r)$, our work on parabolic subgroups suggests that the filtrations classically used in the homology computations for usual braid groups might well be extended to this more general setting.

Before proceeding to the exposition of our main results, we recall the results obtained earlier by $\mathrm{G}$. Lehrer on the rational homology of $B$ for the general series. 
Theorem 1.2 ([Leh04]). The Poincaré polynomial for the cohomology $H^{*}(\mathrm{~B}(e, e, r), \mathbb{Q})$ is

$$
P(\mathrm{~B}(e, e, r), t)= \begin{cases}1+t & \text { if either e or } r \text { is odd, } \\ 1+t+t^{r-1}+t^{r} & \text { otherwise. }\end{cases}
$$

The Poincaré polynomial for the cohomology $H^{*}(\mathrm{~B}(2 e, e, r), \mathbb{Q})$ is

$$
\begin{aligned}
P & (\mathrm{~B}(2 e, e, r), t) \\
& = \begin{cases}(1+t)\left(1+t+t^{2}+\cdots+t^{r-1}\right) & \text { if either e or } r \text { is odd, } \\
(1+t)\left(1+t+t^{2}+\cdots+t^{r-1}\right)+\left(t^{r-1}+t^{r}\right) & \text { otherwise. }\end{cases}
\end{aligned}
$$

\subsection{Main results}

By combining several methods, we are able to compute the low-dimensional integral homology of these groups. We use the notation $\mathbb{Z}_{n}$ or $\mathbb{Z} / n$ for $\mathbb{Z} / n \mathbb{Z}$.

First consider the case of $\mathrm{B}(e, e, r)$. The case $r=2$ is when $G(e, e, 2)$ is a dihedral group, and this case is known by [Sal94]: $H_{2}(B, \mathbb{Z})=0$ if $e$ is odd, and $H_{2}(B, \mathbb{Z})=\mathbb{Z}$ if $e$ is even.

In Section 6 we prove the following result, by using a complex defined by Dehornoy and Lafont [DP99] for Garside monoids and a convenient monoid defined by Corran and Picantin [CP] for the groups $G(e, e, r)$ (of which we prove some additional properties):

Theorem 1.3 (Theorem 6.4). Let $B=\mathrm{B}(e, e, r)$ with $r \geq 3$.

- When $r=3, H_{2}(B, \mathbb{Z}) \simeq \mathbb{Z}_{e}$.

- When $r=4$ and e is odd, $H_{2}(B, \mathbb{Z}) \simeq \mathbb{Z}_{e} \times \mathbb{Z}_{2} \simeq \mathbb{Z}_{2 e}$.

- When $r=4$ and e is even, $H_{2}(B, \mathbb{Z}) \simeq \mathbb{Z}_{e} \times \mathbb{Z}_{2}^{2}$.

- When $r \geq 5, H_{2}(B, \mathbb{Z}) \simeq \mathbb{Z}_{e} \times \mathbb{Z}_{2}$.

In Section 4 (Theorems 4.9,4.15), we compute the homology of complex braid groups of type $\mathrm{B}(2 e, e, r)$ with coefficients in a finite field, using filtrations of the Salvetti complex for the Artin group of type $\mathbf{B}_{r}$ (recall that the homology of this group has first been computed by Goryunov [Gor81]). With a little additional computation (see Section 4.7), we prove as a corollary:

Theorem 1.4. Let $B=\mathrm{B}(2 e, e, r)$ with $r \geq 2$.

- When $r=2$ and e is odd, $H_{2}(B, \mathbb{Z}) \simeq \mathbb{Z}$.

- When $r=2$ and e is even, $H_{2}(B, \mathbb{Z}) \simeq \mathbb{Z}^{2}$.

- When $r=3, H_{2}(B, \mathbb{Z}) \simeq \mathbb{Z}^{2}$.

- When $r=4$ and $e$ is odd, $H_{2}(B, \mathbb{Z}) \simeq \mathbb{Z}^{2} \times \mathbb{Z}_{2}$.

- When $r=4$ and e is even, $H_{2}(B, \mathbb{Z}) \simeq \mathbb{Z}^{2} \times \mathbb{Z}_{2}^{2}$.

- When $r \geq 5, H_{2}(B, \mathbb{Z}) \simeq \mathbb{Z}^{2} \times \mathbb{Z}_{2}$.

We also get a stabilization property for the groups $\mathrm{B}(2 e, e, r)$ similar to the classical one for the usual braid groups (see Corollaries 4.17, 4.18); it turns out that their stable homology does not depend on $e$, and is thus the same as the stable homology of the Artin group of type $\mathbf{B}$. The description of the stable homology is the following: 
Theorem 1.5 (Corollary 4.17). The homology $H_{*}\left(\mathrm{~B}(2 e, e, \infty), \mathbb{F}_{2}\right)$ of the group $\mathrm{B}(2 e, e, \infty)$ is isomorphic to the direct limit $\lim _{r} H_{*}\left(\mathrm{~B}(2 e, e, r), \mathbb{F}_{2}\right)$ and is given by

$$
\mathbb{F}_{2}\left[w_{1}, \bar{x}_{1}, \bar{x}_{2}, \bar{x}_{3}, \ldots\right]
$$

where $\operatorname{dim} w_{1}=1$ and $\operatorname{dim} \bar{x}_{i}=2^{i}-1$. Moreover the canonical morphism to the direct limit

$$
H_{i}\left(\mathrm{~B}(2 e, e, r), \mathbb{F}_{2}\right) \rightarrow H_{i}\left(\mathrm{~B}(2 e, e, \infty), \mathbb{F}_{2}\right)
$$

is an isomorphism for $r>2 i$.

Theorem 1.6 (Corollary 4.18). For $p$ an odd prime, the homology $H_{*}\left(\mathrm{~B}(2 e, e, \infty), \mathbb{F}_{p}\right)$ is isomorphic to the direct limit $\lim _{r} H_{*}\left(\mathrm{~B}(2 e, e, r), \mathbb{F}_{p}\right)$ and is given by the tensor product of

$$
\mathbb{F}_{p}\left[w_{1}, \bar{y}_{1}, \bar{y}_{2}, \bar{y}_{3}, \ldots\right]
$$

and the exterior algebra

$$
\Lambda\left[\bar{x}_{0}, \bar{x}_{1}, \bar{x}_{2}, \ldots\right] .
$$

Here $\operatorname{dim} w_{1}=1, \operatorname{dim} \bar{x}_{i}=2 p^{i}-1$ and $\operatorname{dim} \bar{y}_{i}=2 p^{i}-2$. Moreover the canonical morphism to the direct limit

$$
H_{i}\left(\mathrm{~B}(2 e, e, r), \mathbb{F}_{p}\right) \rightarrow H_{i}\left(\mathrm{~B}(2 e, e, \infty), \mathbb{F}_{p}\right)
$$

is an isomorphism for $r>(i-1) \frac{p}{p-1}+2$.

Theorem 1.7 (Proposition 4.24). For any e the homology $H_{*}(\mathrm{~B}(2 e, e, \infty), \mathbb{Z})$ is isomorphic to the direct limit $\lim _{r} H_{*}(\mathrm{~B}(2 e, e, r), \mathbb{Z})$ and is given by

$$
H_{*}(\mathrm{~B}(2 e, e, \infty), \mathbb{Z}) \simeq H_{*}(\mathrm{~B}(2,1, \infty), \mathbb{Z}) \simeq H_{*}\left(\Omega^{2} S^{3} \times \Omega S^{1}, \mathbb{Z}\right) .
$$

In particular the integral homology of $\mathrm{B}(2 e, e, \infty)$ has no $p^{2}$-torsion. Moreover the canonical morphism to the direct limit

$$
H_{i}(\mathrm{~B}(2 e, e, r), \mathbb{Z}) \rightarrow H_{i}(\mathrm{~B}(2 e, e, \infty), \mathbb{Z})
$$

is an isomorphism for $r>2 i+2$.

Unfortunately, these computations do not suffice in general to get the full integral homology groups. Indeed, we show in Section 4.7 that, in contrast to what happens for Artin groups, the integral homology groups may contain $p^{2}$-torsion. This phenomenon appears for the exceptional groups as well.

The reader will notice that the cell complex that we use for the $G(e, e, r)$, obtained by combining the Dehornoy-Lafont complex and the Corran-Picantin monoid, shares similarities with the Salvetti complex, and actually specializes to it for the usual braid group, in the case $e=1$. It is then likely that this complex can be filtered by a chain of parabolic subcomplexes, paving the way for the methods we use here for the groups $\mathrm{B}(2 e, e, r)$ in order to get the higher homology groups. The differential of the complex is inherited from the work of Kobayashi [Kob90]. The problem is that the behaviour of this 
differential under the simplest operations, like taking the direct product of two monoids or restricting to a parabolic submonoid, is not yet understood. As a consequence, plausible analogues of formulas of the form ' $\overline{\partial 0} A=(\partial A) B+(-1)^{|A|} A 0(\partial B)$ ' (see Section 4.1) are hard to prove.

In Section 7 we compute the integral homology for all exceptional groups, except for $G_{34}$, for which we are able to compute only $H_{2}(B, \mathbb{Z})$ and $H_{3}(B, \mathbb{Z}$ ) (see Table $11)$. As a consequence, we get a complete determination of the groups $H_{2}(B, \mathbb{Z})$ for all complex braid groups. Notice that, since $H_{1}(B, \mathbb{Z})$ is a finitely generated free $\mathbb{Z}$-module, $H_{2}(B, \mathbb{Z})$ determines the cohomology group $H^{2}\left(B, \mathbb{C}^{\times}\right) \simeq \operatorname{Hom}\left(H_{2}(B, \mathbb{Z}), \mathbb{C}^{\times}\right)$, which contains the relevant obstruction classes to the linearization of the projective representations of $B$ - and thus deserves the name 'Schur multiplier' usually restricted to the theory of finite groups. We show in Section 7.1 that the Schur multiplier of $B$ always contains the Schur multiplier of $W$, and that this latter group can most of the time be identified to the 2-torsion subgroup of $\mathrm{H}_{2}(B, \mathbb{Z})$.

Finally, at least when $W$ has one conjugacy class of hyperplanes, there is a welldefined sign morphism $\epsilon: W \rightarrow\{ \pm 1\}$ and a corresponding sign representation $\mathbb{Z}_{\epsilon}$. We determine in general the group $H_{1}\left(B, \mathbb{Z}_{\epsilon}\right)$, which is closely related to the abelianization of the group Ker $\epsilon$ of 'even braids', whose structure remains largely unexplored in general.

Remark 1.8. It should be noted that even the rational homology is not yet known for $W=G_{34}$, due to the large size of $W$ and of its large rank. For instance, formulas involving the lattice (like [OT92, Cor. 6.17]) seem to fail because of the size of the hyperplane arrangement. The methods of [Leh04] could lead to the (possibly computer-aided) counting of points in some $\mathbb{F}_{p}^{6}$, but only if we can get a nice form of the discriminant equation, for which we are able to decide which primes $p$ do satisfy the arithmetic-geometric requirements of [Leh04]. As far as we know, this problem has not been settled yet. Another method would be to use [Leh95], which provides information on $H^{*}(P, \mathbb{Q})$ as a $G_{34}$ module. Indeed, the methods of [Leh95] enable one to compute the trace of the reflections and of so-called regular elements on this module, but it is so huge (the Poincare polynomial of $P$ is $1+126 t+6195 t^{2}+148820 t^{3}+1763559 t^{4}+8703534 t^{5}+7082725 t^{6}$ ) that this does not allow one to determine the dimensions of the invariant subspaces leading to $H^{*}(B, \mathbb{Q})$.

\subsection{Distinction of complex braid groups}

As we noticed before, we can assume that $W$ is a 2-reflection group. We recall that, under the Shephard-Todd parametrization, we have the duplication $G(1,1,4)=G(2,2,3)$. Also notice that the groups $B$ originating from irreducible groups $W$ are distinguishable from the groups originating from non-irreducible ones by the property $Z(B)=\mathbb{Z}$ (see [DMM11, Theorem 1.1]).

It has been noted by Bannai that $G_{13}$ and $G(6,6,2)$ have the same braid group, and that the $\mathrm{B}(2 e, e, 2)$ depend only on the parity of $e$. In [Ban76] it is stated without proof (see Remark 6 there) that these are the only coincidences in rank 2 . This gives a complete classification of isomorphism types for braid groups of rank 2 . We provide a proof that uses our computations. 
Proposition 1.9. On irreducible 2-reflection groups of rank 2, the Bannai isomorphisms are the only coincidences under $W \mapsto B$.

Proof. According to our results, $H_{2}(B, \mathbb{Z})$ is a free $\mathbb{Z}$-module of rank 0,1 or 2 . The case $H_{2}(B, \mathbb{Z})=\mathbb{Z}^{2}$ holds only for the $\mathrm{B}(2 e, e, 2)$ with $e$ even. If $H^{2}(B, \mathbb{Z})=\mathbb{Z}$, then either it is $\mathrm{B}(2 e, e, r)$, or it is a group $\mathrm{B}(e, e, 2)$ with $e$ even. The fact that the groups $\mathrm{B}(e, e, 2)$, that is, the Artin groups of type $I_{2}(e)$, are distinct groups is proved in [Par04], and $\mathrm{B}(2 e, e, 2)$ is the only group of rank 2 with $H_{1}(B, \mathbb{Z})=\mathbb{Z}^{3}$. If $H^{2}(B, \mathbb{Z})=0$, then $W$ is either $G_{12}, G_{22}$ or $G(e, e, 2)$ with $e$ odd. In these cases, there is only one nontrivial morphism $\epsilon: B \rightarrow \mathbb{Z}_{2}$, so we can compare the groups $H_{1}\left(B, \mathbb{Z}_{\epsilon}\right)$ determined in Section 7.2. They are $\mathbb{Z}_{3}$ for $G_{12}, 0$ for $G_{22}$, and $\mathbb{Z}$ for the $\mathrm{B}(e, e, 2)$. Once again, the groups $\mathrm{B}(e, e, 2)$ can be distinguished following [Par04], which concludes the proof.

In order to distinguish the exceptional groups, we need to prove a couple of independent results by ad-hoc methods. We let $B_{23}, B_{24}, \ldots$ denote the complex braid groups associated to $G_{23}, G_{24}, \ldots$.

Lemma 1.10. (1) There is no surjective morphism from $B_{24}$ to the alternating group $\mathfrak{A}_{5}$.

(2) $B_{24}$ is not isomorphic to $B_{23}$.

(3) There is no surjective morphism from $\mathrm{B}(3,3,4)$ to the symmetric group $\mathfrak{S}_{6}$.

(4) $B_{31}$ is not isomorphic to $\mathrm{B}(3,3,4)$.

Proof. Recall that $B_{24}$ has a presentation with generators $s, t, u$ and relations $s t s t=t s t s$, tutu $=u t u t$, sus $=$ usu, tstustu = stustus. We check by computer that none of the $60^{3}$ tuples $(s, t, u) \in \mathfrak{A}_{5}^{3}$ can generate $\mathfrak{A}_{5}$ and satisfy these relations at the same time, which proves (1). This implies (2), as $G_{23} / Z\left(G_{23}\right) \simeq \mathfrak{A}_{5}$ (see [BMR98]). We proceed in the same way for (3), using the presentation in [BMR98] for B (3, 3, 4), namely with generators $s, t, u, v$ and relations sts $=t s t$, stustu $=u s t u s t, s u s=u s u, t u t=$ $u t u, v u v=u v u, v s=s v, v t=t v$. By computer we find that there exist 9360 4-tuples in $\mathfrak{S}_{6}$ satisfying these relations, none of them generating $\mathfrak{S}_{6}$, which proves (3). Then (4) is a consequence of (3), because $G_{31} / Z\left(G_{31}\right)$ admits for quotient $\mathfrak{S}_{6}$ (contrary to what is stated in [BMR98], it is actually a non-split extension of $\mathfrak{S}_{6}$ by a 2-subgroup of order 16; see [Bon02] for more details)

In rank at least 3 , using $H_{2}(B, \mathbb{Z})$ and $H_{1}(B, \mathbb{Z})$, we can separate the groups $\mathrm{B}(2 e, e, r)$ from the rest, as they are the only groups with $H_{1}(B, \mathbb{Z})=\mathbb{Z}^{2}$ and infinite $H_{2}(B, \mathbb{Z})$. All exceptional groups of rank at least 3 have $|\mathcal{A} / W|=1$, that is, $H_{1}(B, \mathbb{Z})=\mathbb{Z}$, except $G_{28}=F_{4}$.

Theorem 1.11. The correspondence $W \mapsto B$ is injective on the 2-reflection groups with a single conjugacy class of reflections, that is, with $|\mathcal{A} / W|=1$.

Proof. Note that the assumption $|\mathcal{A} / W|=1$, which is equivalent to $H_{1}(B, \mathbb{Z})=\mathbb{Z}$, implies that $W$ is irreducible. It also implies that there exists a unique surjective morphism $\epsilon: B \rightarrow \mathbb{Z}_{2}$, so that $H_{1}\left(B, \mathbb{Z}_{\epsilon}\right)$ is well-defined. In rank 2 , the statement to prove is a consequence of the above, so we can assume that the rank $r$ is at least 3 . Then the only cases with infinite $H_{2}(B, \mathbb{Z})$ are the exceptional rank 3 groups $G_{23}, G_{24}, G_{27}$. The $H_{2}$ 
being in these cases $\mathbb{Z}, \mathbb{Z}, \mathbb{Z}_{3} \times \mathbb{Z}$, only $G_{23}$ and $G_{24}$ need to be distinguished, and this is done in Lemma 1.10.

We can now assume that $H_{2}(B, \mathbb{Z})$ is finite. Since all exceptional groups have been taken care of in rank 3 , and $H_{2}(\mathrm{~B}(e, e, 3), \mathbb{Z})=\mathbb{Z}_{e}$ and $H_{2}(\mathrm{~B}(1,1,4), \mathbb{Z})=\mathbb{Z}_{2}$ with $G(1,1,4) \simeq G(2,2,3), W \mapsto B$ is injective in rank 3 and we can assume that the rank is at least 4 . In rank 4 and for $W=G(e, e, 4), e$ is odd exactly when $H_{2}(B, \mathbb{Z})$ is cyclic, so all such $\mathrm{B}(e, e, 4)$ are distinguished by $H_{2}(B, \mathbb{Z})$. Moreover, since $H_{2}\left(B_{29}, \mathbb{Z}\right)=\mathbb{Z}_{2} \times \mathbb{Z}_{4}$ is neither cyclic nor isomorphic to a group of the form $\mathbb{Z}_{e} \times \mathbb{Z}_{2}^{2}$, it does not appear as the $H_{2}$ of a B $(e, e, 4)$. We have $H_{2}\left(B_{30}, \mathbb{Z}\right)=\mathbb{Z}_{2} \simeq \mathbb{Z}_{e} \times \mathbb{Z}_{2}$ if $e=1$, but $G(1,1,4) \simeq \mathfrak{S}_{4}$ has rank 3 . We thus only need to distinguish $B_{31}$ from $\mathrm{B}(3,3,4)$. This can be done either by using $H_{1}\left(B, \mathbb{Z}_{\epsilon}\right)$ or by using the argument in Lemma 1.10 above. When $r \geq 5$, we have $H_{2}(\mathrm{~B}(e, e, r), \mathbb{Z})=\mathbb{Z}_{e} \times \mathbb{Z}_{2}$, and $H_{1}\left(B, \mathbb{Z}_{\epsilon}\right)=\mathbb{Z}_{3}$ when $e \geq 2$. Now $H_{1}\left(B_{33}, \mathbb{Z}_{\epsilon}\right)=$ $H_{1}\left(B_{34}, \mathbb{Z}_{\epsilon}\right)=0, H_{2}\left(B_{33}, \mathbb{Z}\right)=H_{1}\left(B_{34}, \mathbb{Z}\right)=\mathbb{Z}_{6}$, so this distinguishes $B_{33}$ and $B_{34}$. It only remains to distinguish the Artin groups $B_{35}, B_{36}, B_{37}$ of types $E_{6}, E_{7}, E_{8}$ from the usual braid groups $\mathrm{B}(1,1, r)$, and this is done in [Par04].

In the family of groups $\mathrm{B}(2 e, e, r)$, there are many isomorphisms, and we only get partial results in Section 2.

For the convenience of the reader we summarize as a theorem the results that we have obtained about the classification of complex braid groups.

Theorem 1.12. For any finite complex reflection group $W$ the associated complex braid group $B$ is a product of the following groups:

$$
\begin{gathered}
\mathrm{B}_{12}, \mathrm{~B}_{22}, \mathrm{~B}_{23}, \mathrm{~B}_{24}, \mathrm{~B}_{27}, \mathrm{~B}_{28}, \mathrm{~B}_{29}, \mathrm{~B}_{30}, \mathrm{~B}_{31}, \mathrm{~B}_{33}, \mathrm{~B}_{34}, \\
\mathrm{~B}(e, e, r) \text { for } r \geq 2,(e, r) \neq(2,3), B(2 e, e, r) .
\end{gathered}
$$

Moreover the groups from this list belong to the following classes:

(a) $\mathrm{B}(1,1,2)=\mathbb{Z}$ is the only cyclic group;

(b) the groups $\mathrm{B}_{12}, \mathrm{~B}_{22}, \mathrm{~B}_{23}, \mathrm{~B}(e, e, 2)$ for $e \geq 2, \mathrm{~B}(2 e, e, 2)$ for $e=1,2$ are the only groups of homological dimension 2 and are all pairwise non-isomorphic; for general $e$ the groups $\mathrm{B}(2 e, e, 2)$ belong to this list as $\mathrm{B}(2 e, e, 2)=\mathrm{B}(2,1,2)$ for $e$ odd and $\mathrm{B}(2 e, e, 2)=\mathrm{B}(4,2,2)$ for e even;

(c) the groups $\mathrm{B}_{12}, \mathrm{~B}_{22}, \mathrm{~B}_{23}, \mathrm{~B}_{24}, \mathrm{~B}_{27}, \mathrm{~B}_{29}, \mathrm{~B}_{30}, \mathrm{~B}_{31}, \mathrm{~B}_{33}, \mathrm{~B}_{34}, \mathrm{~B}(e, e, r)$ except the case $r=2$ and e even are associated to reflection groups with $|\mathcal{A} / W|=1$ and are characterized by $H_{1}(B, \mathbb{Z})=\mathbb{Z}$; they are pairwise non-isomorphic;

(d) the groups $\mathrm{B}_{28}, \mathrm{~B}(2 e, e, r), r \geq 3$, are the groups with homological dimension $\geq 3$ associated to reflection groups with $|\mathcal{A} / W|=2$, that is, with $H_{1}(B, \mathbb{Z})=\mathbb{Z}^{2}$; they are all distinct from the groups in the lists (a), (b) and (c); moreover we know that $\mathrm{B}(2 e, e, r) \simeq \mathrm{B}\left(2 e^{\prime}, e^{\prime}, r\right)$ for $e \equiv \pm e^{\prime} \bmod r$, and $\mathrm{B}(2 e, e, r) \neq \mathrm{B}\left(2 e^{\prime}, e^{\prime}, r^{\prime}\right)$ for $r \neq r^{\prime}$ and for $r \wedge e \neq r^{\prime} \wedge e^{\prime}$; finally $\mathrm{B}_{28}$ is not isomorphic to any of the groups $\mathrm{B}(2 e, e, r)$.

Proof. (a) is clear because it is the only case of homological dimension 1; (b) comes from Proposition 1.9, (c) from Theorem 1.11; for (d) see Section 2 for the isomorphisms 
between the $\mathrm{B}(2 e, e, r)$ 's, and see the low-dimensional homology computed in Tables 3 and 12 to distinguish $\mathrm{B}_{28}$ from the $\mathrm{B}(2 e, e, r)$.

It is still an open problem whether two groups $\mathrm{B}(2 e, e, r)$ and $\mathrm{B}\left(2 e^{\prime}, e^{\prime}, r\right)$ with $e \not \equiv$ $\pm e^{\prime} \bmod r$ and $r \wedge e=r \wedge e^{\prime}$ are isomorphic or not.

\section{Isomorphism and non-isomorphism results for $\mathrm{B}(2 e, e, r)$}

In this section we study the groups of type $\mathrm{B}(d e, e, r)$ for $d>1$ in order to get some isomorphism and non-isomorphism results.

According to [BMR98], for $d>1$ the group $\mathrm{B}(d, 1, r)$ is the subgroup of the classical braid group

$$
\left.\operatorname{Br}(r+1)=\left\langle\xi_{1}, \ldots, \xi_{r}\right| \xi_{i} \xi_{i+1} \xi_{i}=\xi_{i+1} \xi_{i} \xi_{i+1}, \xi_{i} \xi_{j}=\xi_{j} \xi_{i} \text { if }|i-j| \neq 1\right\rangle
$$

generated by the elements $\xi_{1}^{2}, \xi_{2}, \ldots, \xi_{r}$. This is isomorphic to the Artin group of type $\mathbf{B}_{r}$, $\mathrm{A}_{\mathbf{B}_{r}}$, with corresponding generators $\bar{\sigma}_{1}, \sigma_{2}, \ldots, \sigma_{r}$ and Dynkin diagram as in Table 1.

Table 1. Dynkin diagram for the Artin group of type $\mathbf{B}_{r}$.

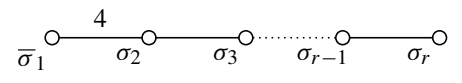

The group $\mathrm{B}(d e, e, r)$ is isomorphic to the subgroup of $\mathrm{B}(d, 1, r)$ generated by

$$
\xi_{1}^{2 e}, \xi_{1}^{2} \xi_{2} \xi_{1}^{-2}, \xi_{2}, \ldots, \xi_{r},
$$

hence it corresponds to the kernel of the map

$$
\phi_{e, r}: \mathrm{A}_{\mathbf{B}_{r}} \rightarrow \mathbb{Z} / e, \quad\left\{\begin{array}{l}
\bar{\sigma}_{1} \mapsto 1, \\
\sigma_{i} \mapsto 0 \text { for } i>1 .
\end{array}\right.
$$

We can give another presentation for $\mathrm{A}_{\mathbf{B}_{r}}$ (see [IP02]). We define $\tau=\bar{\sigma}_{1} \sigma_{2} \cdots \sigma_{r}$ and $\sigma_{1}=\tau^{-1} \sigma_{2} \tau$. It is easy to check that

$$
\tau \sigma_{i} \tau^{-1}=\sigma_{i+1}
$$

where the indices are considered in $\mathbb{Z} / r$.

The group $\mathrm{A}_{\mathbf{B}_{r}}$ has a presentation with generators $\mathcal{G}=\left\{\tau, \sigma_{i}: i \in \mathbb{Z} / r\right\}$ and relations

$$
\mathcal{R}=\left\{\sigma_{i} \sigma_{j}=\sigma_{j} \sigma_{i} \text { for } i \neq j \pm 1, \sigma_{i} \sigma_{i+1} \sigma_{i}=\sigma_{i+1} \sigma_{i} \sigma_{i+1}, \tau \sigma_{i} \tau^{-1}=\sigma_{i+1}\right\}
$$

With this presentation the map $\phi_{e, r}$ maps $\tau \mapsto 1, \sigma_{i} \mapsto 0$ for all $i$.

We notice that the subgroup of $\mathrm{A}_{\mathbf{B}_{r}}$ generated by the elements $\sigma_{1}, \ldots, \sigma_{r}$ is the Artin group of type $\widetilde{A}_{r-1}, \mathrm{~A}_{\tilde{A}_{r-1}}$. Hence, if we write $\mathbb{Z}_{\tau^{e}}$ for the infinite cyclic group generated by $\tau^{e}$, where $\tau$ acts on $\mathrm{A}_{\tilde{A}_{r-1}}$ as before, we can write the following semidirect product decompositions:

$$
\mathrm{B}(d, 1, r)=\mathbb{Z}_{\tau} \ltimes \mathrm{A}_{\tilde{A}_{r-1}}
$$

and

$$
\mathrm{B}(d e, e, r)=\mathbb{Z}_{\tau^{e}} \ltimes \mathrm{A}_{\tilde{A}_{r-1}} .
$$


According to [IP02] and [BMR98] the centre of $\mathrm{B}(d e, e, r)$ is generated by $\beta(d e, e, r)$ $=\left(\tau^{e}\right)^{r / r \wedge e}$. Hence it follows that in the quotient $\mathrm{B}(d e, e, r) / Z(\mathrm{~B}(d e, e, r))$ there is an element, namely $\left(\tau^{e}\right)$, that has order at most $r / r \wedge e$ and is the image of a root of the generator of the centre of $\mathrm{B}(d e, e, r)$. Now let us consider the map

$$
\lambda: \mathrm{B}(d e, e, r) \rightarrow \mathbb{Z} /(r / r \wedge e)
$$

given by $\left(\tau^{e}\right) \mapsto 1$ and $\sigma_{i} \rightarrow 1$ for all $i$. This map passes to the quotient

$$
\bar{\lambda}: \mathrm{B}(d e, e, r) / Z(\mathrm{~B}(d e, e, r)) \rightarrow \mathbb{Z} /(r / r \wedge e)
$$

and hence the order of $\left(\tau^{e}\right)$ in the quotient $\mathrm{B}(d e, e, r) / Z(\mathrm{~B}(d e, e, r))$ is exactly $r / r \wedge e$. The length function in $\mathrm{B}(d e, e, r)$ tells us also that the generator $\beta(d e, e, r)$ of the centre cannot have roots of order higher than $r / r \wedge e$. We have proved the following:

Proposition 2.1. The groups $\mathrm{B}(d e, e, r)$ and $\mathrm{B}\left(d e^{\prime}, e^{\prime}, r\right)$ are not isomorphic if

$$
r \wedge e \neq r \wedge e^{\prime}
$$

From equation (1) it is possible to deduce the following elementary result:

Proposition 2.2. The group $\mathrm{B}(2 e, e, r)$ is isomorphic to $\mathrm{B}\left(2 e^{\prime}, e^{\prime}, r\right)$ if $e \equiv \pm e^{\prime} \bmod r$. Proof. This is straightforward since the Dynkin diagram of $\widetilde{A}_{r-1}$ is an $r$-gon and we can suppose without loss of generality that the vertices are numbered counterclockwise. Hence the element $\tau$ acts by conjugation rotating the $r$-gon by $2 \pi / r$, and the subgroup of $\operatorname{Inn}\left(\mathrm{A}_{\tilde{A}_{r-1}}\right)$ generated by $\tau$ is cyclic of order $r$. It follows that conjugation by $\tau^{e}$ is equivalent to conjugation by $\tau^{e^{\prime}}$ if $e \equiv e^{\prime} \bmod r$. Moreover we can consider the automorphism $\varsigma$ of $\mathrm{A}_{\widetilde{A}_{r-1}}$ given by $\varsigma\left(\sigma_{i}\right)=\sigma_{r+1-i}$. The map $\varsigma$ induces an isomorphism

$$
\bar{\zeta}: \mathbb{Z}_{\tau^{e}} \ltimes \mathrm{A}_{\widetilde{A}_{r-1}} \rightarrow \mathbb{Z}_{\tau^{-e}} \ltimes \mathrm{A}_{\widetilde{A}_{r-1}}
$$

given by $\bar{\varsigma}(n, w)=(-n, \varsigma(w))$.

\section{Homology of the classical braid group}

In what follows we often deal with bigraded modules $M=\bigoplus_{n, d} M_{n, d}$. The first grading function is denoted by deg and refers to an indexed family of objects. The second grading function is denoted by dim and refers to homological dimension. We write $M_{n, d}=M_{\mathrm{deg}=n, \operatorname{dim}=d}$ for the corresponding homogeneous summand of $M$ with fixed degree and dimension.

Let $\operatorname{Br}(n)$ be the classical Artin braid group on $n$ strands. We recall the description of the homology of these groups according to the results of [Coh76, Fuk70, Vaí78]. We shall adopt the notation coherent with [DPS01] (see also [Cal06]) for the description of the algebraic complex and the generators. Let $\mathbb{F}$ be a field. The direct sum of the homology of $\operatorname{Br}(n)$ for $n \in \mathbb{N}=\mathbb{Z}_{\geq 0}$ is considered as a bigraded ring $\bigoplus_{d, n} H_{d}(\operatorname{Br}(n), \mathbb{F})$ where the product structure

$$
H_{d_{1}}\left(\operatorname{Br}\left(n_{1}\right), \mathbb{F}\right) \times H_{d_{2}}\left(\operatorname{Br}\left(n_{2}\right), \mathbb{F}\right) \rightarrow H_{d_{1}+d_{2}}\left(\operatorname{Br}\left(n_{1}+n_{2}\right), \mathbb{F}\right)
$$

is induced by the map $\operatorname{Br}\left(n_{1}\right) \times \operatorname{Br}\left(n_{2}\right) \rightarrow \operatorname{Br}\left(n_{1}+n_{2}\right)$ that juxtaposes braids (see [Coh88, Cal06]). 


\subsection{Braid homology over $\mathbb{Q}$}

The homology of the braid group with rational coefficients has a very simple description:

$$
H_{d}(\operatorname{Br}(n), \mathbb{Q})=\left(\mathbb{Q}\left[x_{0}, x_{1}\right] /\left(x_{1}^{2}\right)\right)_{\mathrm{deg}=n, \operatorname{dim}=d}
$$

where $\operatorname{deg} x_{i}=i+1$ and $\operatorname{dim} x_{i}=i$. In the Salvetti complex for the classical braid group (see [Sal94, DPS01]) the element $x_{0}$ is represented by the string 0 , and $x_{1}$ is represented by the string 10 . In the representation of a monomial $x_{0}^{a} x_{1}^{b}$ we drop the last 0 .

For example the generator of $H_{1}(\operatorname{Br}(4), \mathbb{Q})$ is the monomial $x_{0}^{2} x_{1}$ and we can also write it as a string in the form 001 (instead of 0010, dropping the last 0 ).

We denote by $A(\mathbb{Q})$ the module $\mathbb{Q}\left[x_{0}, x_{1}\right] /\left(x_{1}^{2}\right)\left[t^{ \pm 1}\right]$.

\subsection{Braid homology over $\mathbb{F}_{2}$}

With coefficients in $\mathbb{F}_{2}$ we have

$$
H_{d}\left(\operatorname{Br}(n), \mathbb{F}_{2}\right)=\mathbb{F}_{2}\left[x_{0}, x_{1}, x_{2}, x_{3}, \ldots\right]_{\mathrm{deg}=n, \operatorname{dim}=d}
$$

where the generator $x_{i}, i \in \mathbb{N}$, has degree $\operatorname{deg} x_{i}=2^{i}$ and homological dimension $\operatorname{dim} x_{i}=2^{i}-1$.

In the Salvetti complex the element $x_{i}$ is represented by a string of $2^{i}-11$ 's followed by one 0 . In the representation of a monomial $x_{i_{1}} \ldots x_{i_{k}}$ we drop the last 0 .

We denote by $A\left(\mathbb{F}_{2}\right)$ the module $\mathbb{F}_{2}\left[x_{0}, x_{1}, x_{2}, x_{3}, \ldots\right]\left[t^{ \pm 1}\right]$.

\subsection{Braid homology over $\mathbb{F}_{p}, p>2$}

With coefficients in $\mathbb{F}_{p}$ with $p$ an odd prime, we have

$$
H_{d}\left(\operatorname{Br}(n), \mathbb{F}_{p}\right)=\left(\mathbb{F}_{p}\left[h, y_{1}, y_{2}, y_{3}, \ldots\right] \otimes \Lambda\left[x_{0}, x_{1}, x_{2}, x_{3}, \ldots\right]\right)_{\operatorname{deg}=n, \operatorname{dim}=d}
$$

where the second factor in the tensor product is the exterior algebra over the field $\mathbb{F}_{p}$ with generators $x_{i}, i \in \mathbb{N}$. The generator $h$ has degree $\operatorname{deg} h=1$ and homological dimension $\operatorname{dim} h=0$. The generator $y_{i}, i \in \mathbb{N}$, has $\operatorname{deg} y_{i}=2 p^{i}$ and $\operatorname{dim} y_{i}=2 p^{i}-2$. The generator $x_{i}, i \in \mathbb{N}$ has $\operatorname{deg} x_{i}=2 p^{i}$ and $\operatorname{dim} x_{i}=2 p^{i}-1$.

In the Salvetti complex the element $h$ is represented by the string 0 , the element $x_{i}$ is represented by a string of $2^{i}-11$ 's followed by one 0 . We remark that the term $d\left(x_{i}\right)$ is divisible by $p$. In fact, with generic coefficients (see [Cal06]), the differential $d\left(x_{i}\right)$ is given by a sum of terms with coefficients all divisible by the cyclotomic polynomial $\varphi_{2 p^{i}}(q)$. Specializing to the trivial local system with integer coefficients, all terms are divisible by $\varphi_{2 p^{i}}(-1)=p$. The element $y_{i}$ is represented by the following term (the differential is computed over the integers and then, after dividing by $p$, we consider the result modulo $p$ ):

$$
\frac{d\left(x_{i}\right)}{p}
$$

Hence we can associate a string to a monomial $x_{i_{1}} \cdots x_{i_{k}} h^{i} y_{j_{1}} \cdots y_{j_{l}}$ juxtaposing the strings associated to each single factor and dropping the last 0 . Here the indices vary with $i_{1}<\cdots<i_{k}$ and $j_{1} \leq \cdots \leq j_{l}$. 
We denote by $A\left(\mathbb{F}_{p}\right)$ the module

$$
\mathbb{F}_{p}\left[h, y_{1}, y_{2}, y_{3}, \ldots\right] \otimes \Lambda\left[x_{0}, x_{1}, x_{2}, x_{3}, \ldots\right]\left[t^{ \pm 1}\right] .
$$

We write simply $A$ instead of $A(\mathbb{Q}), A\left(\mathbb{F}_{2}\right)$ or $A\left(\mathbb{F}_{p}\right)$ when the field we are considering is understood.

\section{Homology of $\mathrm{B}(2 e, e, r)$}

\subsection{Preliminary computations}

In this section we always assume $d>1$. Recall (from [BMR98]) that in that case we have $\mathrm{B}(d e, e, r)=\mathrm{B}(2 e, e, r)$. The case $d=1$ will be treated in Section 6.

We want to understand the homology of $\mathrm{B}(* e, e, r)=\mathrm{B}(2 e, e, r)$ with coefficients in $\mathbb{F}_{p}$.

We start by computing the homology of the group $\mathrm{B}(2 e, e, r)$ with coefficients in the field $\mathbb{F}$. In what follows $\mathbb{F}$ will be mainly a prime field $\mathbb{F}_{p}$, but we will also be interested to obtain again the results of Lehrer for rational coefficients in order to have a description of the generators.

Let us recall from Section 2 the isomorphisms

$$
\mathrm{B}(d, 1, r)=\mathrm{A}_{\mathbf{B}_{r}} \quad \text { and } \quad \mathrm{B}(d e, e, r)=\operatorname{Ker} \phi_{e, r}
$$

where $\phi_{e, r}$ maps $\mathrm{B}(d, 1, r) \rightarrow \mathbb{Z} / e$.

We can consider the group rings $\mathbb{F}[\mathbb{Z} / e]=\mathbb{F}[t] /\left(1-(-t)^{e}\right)$ and $\mathbb{F}[\mathbb{Z}]=\mathbb{F}\left[t^{ \pm 1}\right]$ as $\mathrm{A}_{\mathbf{B}_{r}}$-modules through the maps $\phi_{e, r}$ and $\phi_{r}: \mathrm{A}_{\mathbf{B}_{r}} \rightarrow \mathbb{Z}$ that maps $\bar{\sigma}_{1} \mapsto 1$ and $\sigma_{i} \mapsto 0$ for $i>1$.

Applying the Shapiro Lemma (see [Bro82]) we find that

$$
H_{*}(\mathrm{~B}(d e, e, r), \mathbb{F})=H_{*}\left(\mathrm{~A}_{\mathbf{B}_{r}}, \mathbb{F}[t] /\left(1-(-t)^{e}\right)\right) .
$$

Notice that this statement is also true when $\mathbb{F}$ is an arbitrary ring. In order to compute the right term of the equality we begin by studying the homology $H_{*}\left(\mathrm{~A}_{\mathbf{B}_{r}}, \mathbb{F}\left[t^{ \pm 1}\right]\right)$, where the local system is determined by the map $\phi_{r}$. To do this we consider the algebraic Salvetti complex for the Artin group of type $\mathbf{B}_{r}, C_{*}(r)=C_{*}\left(\mathrm{~A}_{\mathbf{B}_{r}}\right)$ (see [Sal94]), with coefficients in the group ring $\mathbb{F}\left[t^{ \pm 1}\right]$. We order the generators of $\mathrm{A}_{\mathbf{B}_{r}}$ as in the diagram of Table 1 . We filter the complex $C_{*}(r)$ as follows:

$$
\mathcal{F}_{i} C_{*}(r)=\langle A B\rangle
$$

where $\langle A B\rangle$ is the $\mathbb{F}\left[t^{ \pm 1}\right]$-submodule of $C_{*}(r)$ generated by all the strings of type $A B$, with $A$ a string of 0 's and 1's of length $i$ with at least one 0 . It follows that we have an isomorphism

$$
\mathcal{F}_{i+1} C_{*}(r) / \mathcal{F}_{i} C_{*}(r) \stackrel{\iota}{\simeq} C_{*}\left(\mathrm{~A}_{\mathbf{A}_{r-i-1}}\right)[i, i+1]
$$

between the quotient of two consecutive filtrated terms and the Salvetti complex for the Artin group of type $\mathbf{A}_{r-i-1}$, that is, the braid group $\operatorname{Br}(r-i)$ on $r-i$ strands. The first index in square brackets means dimension shifting by $i$ and the second index means degree shifting by $i+1$. The complex $\mathcal{F}_{i+1} C_{*}(r) / \mathcal{F}_{i} C_{*}(r)$ is generated by strings of 
the form $1^{i} 0 B$. Moreover the string $1^{i} 0 B$ corresponds, through the isomorphism $\iota$, to the string $B$ in the complex $C_{*}\left(\mathrm{~A}_{\mathbf{A}_{r-i-1}}\right)[i, i+1]$.

We consider the direct sum

$$
C_{*}=\bigoplus_{r=0}^{\infty} C_{*}(r)
$$

and we study the first quadrant spectral sequence $\left\{E_{i, j}^{k}, d^{k}\right\}_{k}$ induced by the filtration $\mathcal{F}$ on the complex $C_{*}$. The complex $C_{*}$ is bigraded with

$$
|S|=\operatorname{dim} S=\text { the number of 1's of the string } S
$$

and

$$
\operatorname{deg} S=\text { the length of the string } S \text {. }
$$

The first observation is that we get a first quadrant spectral sequence and in the $E^{0}$ term we have

$$
E_{i, j}^{0}=\mathcal{F}_{i} C_{i+j} / \mathcal{F}_{i-1} C_{i+j}=\bigoplus_{r=0}^{\infty} C_{j}\left(\mathrm{~A}_{\mathbf{A}_{r-i-1}}\right) .
$$

We can now study the first differential of the spectral sequence, that is, $d^{0}$. Because of the chosen filtration, on each column of the spectral sequence the differential $d^{0}$ corresponds to the boundary map of the complex $C_{*}\left(\mathrm{~A}_{\mathbf{A}_{r-i-1}}\right)$ with trivial local system. This yields

Proposition 4.1. The $E^{1}$ term of the first quadrant spectral sequence defined above is given as follows $(i, j \geq 0)$ :

$$
\begin{aligned}
E_{i, j}^{1} & =\bigoplus_{r=0}^{\infty} H_{j}\left(\mathrm{~A}_{\mathbf{A}_{r-i-1}}, \mathbb{F}\left[t^{ \pm 1}\right]\right)=\bigoplus_{r=0}^{\infty} H_{j}\left(\operatorname{Br}(r-i), \mathbb{F}\left[t^{ \pm 1}\right]\right) \\
& =\bigoplus_{r=0}^{\infty} H_{j}(\operatorname{Br}(r-i), \mathbb{F}) \otimes \mathbb{F}\left[t^{ \pm 1}\right]
\end{aligned}
$$

since the $t$-local system is trivial on $\operatorname{Br}(r-i)$.

Notation: We denote by $\operatorname{Br}(0)$ and $\operatorname{Br}(1)$ the trivial group with one element, while $\operatorname{Br}(i)$ is empty for $i<0$. Hence $H_{*}(\operatorname{Br}(1), \mathbb{F})=H_{*}(\operatorname{Br}(0), \mathbb{F})=\mathbb{F}$ and both modules are concentrated in dimension 0 , while $H_{j}(\operatorname{Br}(i), \mathbb{F})$ is the trivial summand for $i<0$.

Remark 4.2. Proposition 4.1 actually gives us an infinite family of spectral sequences. Using the previous argument we can define, for every index $r \in \mathbb{N}$, a spectral sequence $\left\{E_{i, j}^{k}(r), d^{k}(r)\right\}_{k}$ with first term

$$
E_{i, j}^{1}(r)=H_{j}(\operatorname{Br}(r-i), \mathbb{F}) \otimes \mathbb{F}\left[t^{ \pm 1}\right]
$$

which converges to the homology group $H_{*}\left(\mathrm{~A}_{\mathbf{B}_{r}}, \mathbb{F}\left[t^{ \pm 1}\right]\right)$.

Notice that each column of the $E^{1}$ term of the spectral sequence of Proposition 4.1 is isomorphic to the bigraded module $A(\mathbb{F})$ defined in Section 3. The correspondence between an element $x \in A(\mathbb{F})$ and an element in the $i$-th column of the spectral sequence 
is the following: if $x$ is a monomial, which corresponds to a string of 0's and 1's, we lift it to the same string preceded by the sequence

$$
\overbrace{1 \cdots 1}^{i} 0
$$

For a generic element $x$ we extend the correspondence by linearity. We denote the lifted element by $z_{i} x$.

Our interest now is to study the higher differentials of the spectral sequence. Since they are induced by the boundary map of the complex $C_{*}(r)$, we give a description of this complex according to [Sal94] and [CMS08].

We recall the definition of the following $q$-analog and $q, t$-analog polynomials:

$$
\begin{gathered}
{[0]_{q}:=1, \quad[m]_{q}:=1+q+\cdots+q^{m-1}=\frac{q^{m}-1}{q-1} \quad \text { for } m \geq 1,} \\
{[m]_{q} !:=\prod_{i=1}^{m}[m]_{q}, \quad\left[\begin{array}{c}
m \\
i
\end{array}\right]_{q}:=\frac{[m]_{q} !}{[i]_{q} ![m-i]_{q} !},} \\
{[2 m]_{q, t}:=[m]_{q}\left(1+t q^{m-1}\right), \quad[2 m]_{q, t} ! !:=\prod_{i=1}^{m}[2 i]_{q, t}=[m]_{q} ! \prod_{i=0}^{m-1}\left(1+t q^{i}\right),} \\
{\left[\begin{array}{c}
m \\
i
\end{array}\right]_{q, t}^{\prime}:=\frac{[2 m]_{q, t} ! !}{[2 i]_{q, t} ! ![m-i]_{q} !}=\left[\begin{array}{c}
m \\
i
\end{array}\right]_{q} \prod_{j=i}^{m-1}\left(1+t q^{j}\right) .}
\end{gathered}
$$

In our computations, since we consider a local system that maps the generator associated to the first node of the Dynkin diagram $\mathbf{B}_{r}$ to a non-trivial action (i.e. $(-t)$ multiplication) and the other generators to a trivial action, we will specialize our polynomials to $q=-1$ (see [Sal94]).

By an easy computation with cyclotomic polynomials, combined with some result that appears in [Cal06], we can easily prove the following lemma, which will be useful in further computations. We will write $[*]_{-1}$ for the $q$-analog [*] evaluated at $q=-1$.

Lemma 4.3. For $q=-1$, the polynomial $\left[\begin{array}{c}m \\ i\end{array}\right]_{q}$ evaluated over the integers is zero if and only if $m$ is even and $i$ is odd.

Evaluated modulo 2, it is non-zero if and only if, when we write $i$ and $m$ as sums of powers of 2 without repetitions, all the terms in the sum for $i$ appear in the sum for $m$. Let $h$ be the number of integers $k$ such that there is a 1 in the binary decomposition of $i$ or $m-i$ at the $k$-th position, but not in the binary decomposition for $m$. Then $2^{h}$ is the highest power of 2 that divides the integer $\left[\begin{array}{c}m \\ i\end{array}\right]_{-1}$.

Evaluated modulo a prime $p>2$, the expression $\left[\begin{array}{c}m \\ i\end{array}\right]_{-1}$ is non-zero if and only if when we write $i$ and $m-i$ as sums of terms of the form

$$
i=l_{0}+\sum_{k=1}^{s} l_{k} 2 p^{k-1}, \quad m-i=l_{0}^{\prime}+\sum_{k=1}^{s} l_{k}^{\prime} 2 p^{k-1}
$$


with $0 \leq l_{0}, l_{0}^{\prime} \leq 1,0 \leq l_{k}, l_{k}^{\prime}<$ for $k=1, \ldots, s$, we have $l_{0}+l_{0}^{\prime}<2$ and $l_{k}+l_{k}^{\prime}<p$ for all $k=1, \ldots, s$. Moreover, if

$$
m=l_{0}^{\prime \prime}+\sum_{k=1}^{s} l_{k}^{\prime \prime} 2 p^{k-1}
$$

with $0 \leq l_{0}^{\prime \prime} \leq 1,0 \leq l_{k}^{\prime \prime}<p$ for $k=1, \ldots, s$, then the integer $h$ defined as

$$
h:=\sharp\left\{k \in \mathbb{N} \mid l_{h}^{\prime \prime}<l_{h}+l_{h}^{\prime}\right\}
$$

is the greatest exponent such that $p^{h}$ divides $\left[\begin{array}{c}m \\ i\end{array}\right]_{-1}$.

Proof. Let us sketch the idea of the proof. The main point is to study the divisibility of the polynomial $\left[\begin{array}{c}m \\ i\end{array}\right]_{q}$ by the cyclotomic polynomials $\varphi_{j}(q)$. Moreover we need to recall that $\varphi_{j}(-1) \neq 0$ if $j \neq 2$ and for any prime $p, \varphi_{2 p^{j}}(-1)=p$ for $j>0$, and in all the other cases $\varphi_{j}(-1)=1$. The number $h$ is the number of digits that we carry over in the sum between $i$ and $m-i$ written respectively in base 2 and in the base associated to an odd prime $p$, corresponding to the sums of the last part of the statement. The integer $h$ actually counts the number of times a factor of the form $\varphi_{2 p^{j}}, p>0$, divides the $q$-analog $\left[\begin{array}{c}m \\ i\end{array}\right]_{q}$.

Finally we present the boundary maps for the complex $C_{*}\left(\mathrm{~A}_{\mathbf{A}_{r}}\right)$ and $C_{*}(r)$, which we write $\partial$ and $\bar{\partial}$. Recall that the complex $C_{*}\left(\mathrm{~A}_{\mathbf{A}_{r}}\right)$ over a module $M$ is the direct sum

$$
\bigoplus_{|x|=r} M \cdot x
$$

of one copy of $M$ for each string $x$, made of 0's and 1's, of length $r$. Notice that these strings are in 1-to-1 correspondence with the parts of a set of $r$ elements (in particular with the set of nodes of the Dynkin diagram of type $\mathbf{A}_{r}$ ). A 1 in the $j$-th position of the string means that the $j$-th element belongs to the subset, while a 0 means it does not. The complex is graded as follows: the dimension of a non-zero element $m \in M . x$ is given by the number of 1's in the string $x$, that is, the cardinality of the corresponding subset.

The complex $C_{*}(r)=C_{*}\left(\mathrm{~A}_{\mathbf{B}_{r}}\right)$ has the same description (as a graded module) as $C_{*}\left(\mathrm{~A}_{\mathbf{A}_{r}}\right)$, but they differ in the boundary. Since in the Dynkin diagram of type $\mathbf{B}_{r}$ the first node is special, we change our notation slightly for the string representing the generators of $C_{*}(r)$ using $\overline{0}$ or $\overline{1}$ in the first position, according to whether or not the first element belongs to the subset of nodes.

We consider the nodes of the Dynkin diagram of type $\mathbf{A}_{r}$ ordered as in Table 2.

Table 2. Dynkin diagram for the Artin group of type $\mathbf{A}_{r}$.

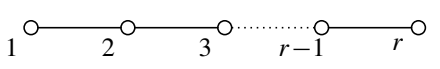

Let $x$ be the string

$$
\overbrace{1 \cdots 1}^{i_{1}} 0 \overbrace{1 \cdots 1}^{i_{2}} 0 \cdots 0 \overbrace{1 \cdots 1}^{i_{k}} .
$$


We write it in more compact notation as

$$
x=1^{i_{1}} 01^{i_{2}} 0 \cdots 01^{i_{k}} .
$$

The boundary of $x$ in the complex $C_{*}\left(\mathrm{~A}_{\mathbf{A}_{r}}\right)$ is given by the following sum:

$$
\partial x=\sum_{j=1}^{k} \sum_{h=0}^{i_{j}-1}(-1)^{i_{1}+\ldots+i_{j-1}+h}\left[\begin{array}{c}
i_{j}+1 \\
h+1
\end{array}\right]_{-1} 1^{i_{1}} 0 \cdots 01^{i_{j-1}} 01^{h} 01^{i_{j}-h-1} 01^{i_{j+1}} 0 \cdots 01^{i_{k}} .
$$

In a simpler way (see [DPS01, DPSS99]) we can say that the boundary is zero on the string made of all 0's; moreover,

$$
\partial 1^{l}=\sum_{h=0}^{l}-1(-1)^{h}\left[\begin{array}{l}
l+1 \\
h+1
\end{array}\right]_{-1} 1^{h} 01^{l-h-1}
$$

and if $A$ and $B$ are two strings,

$$
\partial A 0 B=(\partial A) 0 B+(-1)^{|A|} A 0 \partial B .
$$

In the complex $C_{*}(r)$ the boundary $\bar{\partial} x$ is given as follows:

$$
\begin{aligned}
\bar{\partial} 0 A & =\overline{0} \partial A \\
\bar{\partial} \overline{1} 1^{l-1} & =\left[\begin{array}{l}
l \\
0
\end{array}\right]_{-1}^{\prime} \overline{0} 1^{l-1}+\sum_{h=1}^{l-1}(-1)^{h}\left[\begin{array}{l}
l \\
h
\end{array}\right]_{-1}^{\prime} \overline{1} 1^{h-1} 01^{l-h-1} \\
\bar{\partial} A 0 B & =(\bar{\partial} A) 0 B+(-1)^{|A|} A 0 \partial B .
\end{aligned}
$$

We can use the given description of the algebraic complex to compute explicitly the differential $d^{1}$ in the spectral sequence. This is a first tutorial step in the computation of the whole spectral sequence of Proposition 4.1. Recall that $d^{1}$ is a homomorphism of bidegree $(-1,0)$ and maps

$$
d_{i, j}^{1}(r): E_{i, j}^{1}(r) \rightarrow E_{i-1, j}^{1}(r) .
$$

A representative of a generator of $E_{i, j}^{1}(r)$ is of the form $\overline{1}^{i-1} 0 x=z_{i} x$ where $x$ is a representative of a homology class in $H_{j}(\operatorname{Br}(r-i), \mathbb{F})$. Since $x$ is already a cycle, we need to consider only the part of the boundary $\bar{\partial} 1^{i} 0 x$ starting with $1^{i-1} 0$, that is,

$$
d_{i, j}^{1}(r): z_{i} x \mapsto[i]_{-1}\left(1+t(-1)^{i-1}\right) z_{i-1} 0 x .
$$

Since the coefficient $[i]_{-1}$ is 0 for even $i$ 's and 1 otherwise, we get

$$
d_{i, j}^{1}(r) z_{i} x= \begin{cases}0 & \text { if } i \text { is even } \\ (1+t) z_{i-1} 0 x & \text { if } i \text { is odd. }\end{cases}
$$

When we work on the prime field $\mathbb{F}_{p}$, with $p=2$ we write also $z_{i-1} x_{0} x$ for $z_{i-1} 0 x$ and when $p>2$ we write $z_{i-1} h x$ for $z_{i-1} 0 x$. Each odd column injects in the even column on its left. The $E^{2}$ term of the spectral sequence easily follows from the description of the differential $d^{1}$. We can briefly state this as: 
Proposition 4.4. In the $E^{2}$ term of the first quadrant spectral sequence of Proposition 4.1 each column in even position is isomorphic to the quotient ring $A\left(\mathbb{F}_{2}\right) /\left((1+t) x_{0}\right)$ (resp. $A\left(\mathbb{F}_{p}\right) /((1+t) h)$ or $\left.A(\mathbb{Q})\left((1+t) x_{0}\right)\right)$ for $\mathbb{F}=\mathbb{F}_{2}\left(\right.$ resp. $\mathbb{F}=\mathbb{F}_{p}, p>2$ or $\left.\mathbb{F}=\mathbb{Q}\right)$. All the columns in odd position are zero.

For a more advanced study of the spectral sequence and of its other terms, we need to split our analysis, considering separately the case $\mathbb{F}=\mathbb{Q}$ and the cases $\mathbb{F}=\mathbb{F}_{p}$, with $p=2$ and $p>2$.

\subsection{Homology of $\mathrm{B}(2 e, e, r)$ with rational coefficients}

We continue the study of the spectral sequence of Proposition 4.4. We only need to compute the differential of the $E^{2}$ term of the spectral sequence, since the spectral sequence is concentrated in the first two rows, hence all the other differentials are zero and the spectral sequence collapses at $E^{3}$.

The differential

$$
d_{i, j}^{2}(r): E_{i, j}^{1}(r) \rightarrow E_{i-2, j+1}^{1}(r)
$$

acts as follows:

$$
d_{i, j}^{2}(r): z_{i} x \mapsto\left[\begin{array}{l}
i \\
2
\end{array}\right]_{-1}\left(1-t^{2}\right) z_{i-2} x_{1} x
$$

The coefficient $\left[\begin{array}{l}i \\ 2\end{array}\right]_{-1}$ is always non-zero, hence we can define the quotient

$$
A_{0}(\mathbb{Q})=A_{0}=A(\mathbb{Q}) /\left((1+t) x_{0},\left(1-t^{2}\right) x_{1}\right)
$$

and in the $E^{\infty}$ term we have:

\begin{tabular}{|l|l|l|l|l|l|l}
$A_{0}$ & 0 & $A_{0}$ & 0 & $A_{0}$ & 0 & $\cdots$ \\
\hline
\end{tabular}

The terms of the form $z_{2 i} x_{0}^{j}$ lift, in $H_{*}\left(\mathrm{~A}_{\mathbf{B}_{r}}, \mathbb{Q}\left[t^{ \pm 1}\right]\right)$, to the cycle

$$
\omega_{2 i, j, 0}=\frac{\bar{\partial}\left(z_{2 i+1} x_{0}^{j-1}\right)}{1+t}
$$

while the terms of the form $z_{2 i} x_{0}^{j} x_{1}$ for $j>1$ lift to

$$
\omega_{2 i, j, 1}=\frac{\bar{\partial}\left(z_{2 i+1} x_{0}^{j-1} x_{1}\right)}{1+t}
$$

and for $j=0$ to

$$
\omega_{2 i, 0,1}=\frac{\bar{\partial}\left(z_{2 i+2}\right)}{1-t^{2}} .
$$


We can then compute the homology $H_{*}(\mathrm{~B}(2 e, e, r), \mathbb{Q})=H_{*}\left(\mathrm{~A}_{\mathbf{B}_{r}}, \mathbb{Q}[t] /\left(1-(-t)^{e}\right)\right)$ by means of the homology long exact sequence associated to the short exact sequence

$$
0 \rightarrow \mathbb{Q}\left[t^{ \pm 1}\right] \stackrel{\left(1-(-t)^{e}\right)}{\longrightarrow} \mathbb{Q}\left[t^{ \pm 1}\right] \stackrel{\pi}{\rightarrow} \mathbb{Q}\left[t^{ \pm 1}\right] /\left(1-(-t)^{e}\right) \rightarrow 0 .
$$

We consider the following cycles in the complex for $H_{*}\left(\mathrm{~A}_{\mathbf{B}_{r}}, \mathbb{Q}[t] /\left(1-(-t)^{e}\right)\right)$ :

$$
\begin{aligned}
\bar{\omega}_{2 i, j, 0} & =\frac{\left(1-(-t)^{e}\right) z_{2 i+1} x_{0}^{j-1}}{1+t}, \\
\bar{\omega}_{2 i, j, 1} & =\frac{\left(1-(-t)^{e}\right) z_{2 i+1} x_{0}^{j-1} x_{1}}{1+t}, \\
\bar{\omega}_{2 i, 0,1} & =\frac{\left(1-(-t)^{e}\right) z_{2 i+2}}{1-t^{2}} .
\end{aligned}
$$

Let $\delta$ be the differential of the long exact homology sequence associated to the short exact sequence (2); it is clear that

$$
\delta\left(\bar{\omega}_{2 i, j, k}\right)=\omega_{2 i, j, k}
$$

Moreover the cycles $\bar{\omega}_{2 i, j, k}$ have $(1+t)$-torsion if $(j, k) \neq(0,1)$, and $\left(1-t^{2}\right)$-torsion otherwise. This proves that the cycles $\bar{\omega}$ and $\pi_{*}(\omega)$ are the generators of the homology $H_{*}(\mathrm{~B}(2 e, e, r), \mathbb{Q})$ confirming the Poincaré polynomial already given by Lehrer [Leh04].

\section{3. $H_{*}\left(\mathrm{~A}_{\mathbf{B}_{r}}, \mathbb{F}_{2}\left[t^{ \pm 1}\right]\right)$}

We can now compute the differential in the $E^{2}$ term of the spectral sequence. The boundary map tells us that the differential

$$
d_{i, j}^{2}(r): E_{i, j}^{1}(r) \rightarrow E_{i-2, j+1}^{1}(r)
$$

acts as follows:

$$
d_{i, j}^{2}(r): z_{i} x \mapsto\left[\begin{array}{l}
i \\
2
\end{array}\right]_{-1}(1+t)^{2} z_{i-2} x_{1} x
$$

The coefficient $\left[\begin{array}{l}i \\ 2\end{array}\right]_{-1}$, which we consider only for even values of $i$, is zero if $4 \mid i$, otherwise it is non-zero and the kernel of the differential is generated by the element $x_{0}$. Hence the picture of the spectral sequence for $E^{3}=E^{4}$ (note that the differential $d^{3}$ must be zero) is as follows: if $i$ is a multiple of 4 , then the $i$-th column is isomorphic to the quotient $A\left(\mathbb{F}_{2}\right) /\left((1+t) x_{0},(1+t)^{2} x_{1}\right)$, and if $i$ is even, but $4 \nmid i$, then the $i$-th column is isomorphic to the submodule quotient $x_{0} A\left(\mathbb{F}_{2}\right) /\left((1+t) x_{0}\right) \simeq A\left(\mathbb{F}_{2}\right) /(1+t)$ (this is an isomorphism, but not a bigraded-isomorphism); all the other columns are zero.

In order to give a description of the general behaviour of the spectral sequence we need the following definitions.

For $a \in \mathbb{N}$ we define the following ideals of $A=A\left(\mathbb{F}_{2}\right)$ (also for these definitions we drop the notation referring to the prime $p=2$ when it is understood):

$$
J_{a}\left(\mathbb{F}_{2}\right)=J_{a}=\left((1+t) x_{0},\left(1-t^{2}\right) x_{1}, \ldots,\left(1-t^{2}\right)^{2^{a-1}} x_{a}\right) .
$$


We also define the quotients

$$
A_{a}\left(\mathbb{F}_{2}\right)=A_{a}=A\left(\mathbb{F}_{2}\right) / J_{a}\left(\mathbb{F}_{2}\right)
$$

and the ideals of $A_{a}$ :

$$
I_{a}\left(\mathbb{F}_{2}\right)=I_{a}=\left(x_{0}, x_{1}, \ldots, x_{a}\right) \subset A_{a}\left(\mathbb{F}_{2}\right) .
$$

Moreover we define

$$
J_{\infty}\left(\mathbb{F}_{2}\right)=J_{\infty}=\bigcup_{a=0}^{\infty} J_{a}\left(\mathbb{F}_{2}\right) \quad \text { and } \quad A_{\infty}\left(\mathbb{F}_{2}\right)=A_{\infty}=A / J_{\infty}
$$

With this notation the page $E^{3}=E^{4}$ of the spectral sequence looks as follows:

\begin{tabular}{|l|l|l|l|l|l|l|}
$A_{1}$ & 0 & $I_{0}$ & 0 & $A_{1}$ & 0 & $\cdots$ \\
\hline
\end{tabular}

This result gives a description of the general behaviour of the spectral sequence:

Theorem 4.5. The $k$-th term of the spectral sequence described in Proposition 4.1 computing the homology $H_{*}\left(\mathrm{~A}_{\mathbf{B}_{r}}, \mathbb{F}_{2}\left[t^{ \pm 1}\right]\right)$ is as follows:

- If $k=2^{a}$ the $i$-th column is isomorphic to:

- 0 if $i$ is odd;

$-I_{h}$ if $2^{h+1} \mid i$ and $2^{h+2} \nmid i$, with $h+1<a$;

$-A_{a-1}$ if $2^{a} \mid i$.

The differential $d^{2^{a}}$ is as follows: if $2^{a} \mid i$ and $2^{a+1} \nmid i$ we have the map

$$
d_{i, j}^{k}: z_{i} x \mapsto\left[\begin{array}{c}
i \\
2^{a}
\end{array}\right]_{-1}(1+t)^{2^{a}} z_{i-2^{a} x_{a} x}
$$

where the $q$-analog coefficient is invertible; all the other differentials are trivial.

- If $2^{a}<k<2^{a+1}$ then $E^{k}=E^{2^{a+1}}$ and the differential $d^{k}$ is trivial.

In the $E^{\infty}$ term of the spectral sequence the $i$-th column is isomorphic to:

- 0 if $i$ is odd;

- $I_{h}$ if $2^{h+1} \mid i$ and $2^{h+2} \nmid i$;

- $A_{\infty}$ if $i=0$.

The homology $H_{*}\left(\mathrm{~A}_{\mathbf{B}_{r}}, \mathbb{F}_{2}\left[t^{ \pm 1}\right]\right)$ is isomorphic to the graded module associated to the $E^{\infty}$ term.

Proof. We prove the first part of the statement by induction on $a$. The second part will follow from the first.

We already have a description of the $E^{4}$ term, so we can use $a=2$ as a starting point for the induction. 
In order to prove the inductive step, it is useful to give a more precise statement with an explicit description of the generators in the $E^{k}$ term of the spectral sequence.

Let $2^{a-1}<k \leq 2^{a}$ and let $I_{h}$ be an ideal in the $i$-th column (hence $2^{h+1} \mid i$ and $2^{h+2} \nmid i, h+1<\bar{a}$ ). The generators $x_{0}, \ldots, x_{h}$ of the ideal $I_{h}$ are the images of the elements $z_{i} x_{0}, \ldots, z_{i} x_{h}$ of the $E^{0}$ term of the spectral sequence. A generic monomial of the ideal $I_{h}$ is $m=x_{s} x_{s_{1}} \cdots x_{s_{n}}$ with $0 \leq s \leq h, s \leq s_{1} \leq \cdots \leq s_{n}$. The monomial $m$ is the image of the element $z_{i} x_{s} x_{s_{1}} \cdots x_{s_{n}}$ in the $E^{0}$ term of the spectral sequence. Its lifting to the $E^{k}$ term of the spectral sequence is given by

$$
\alpha_{i, 0}=\frac{\bar{\partial}\left(z_{i+1} x_{s_{1}} \cdots x_{s_{n}}\right)}{1+t}
$$

for $s=0$ and

$$
\alpha_{i, s}=\frac{\bar{\partial}\left(z_{i+2^{s}} x_{s_{1}} \cdots x_{s_{n}}\right)}{\left(1-t^{2}\right)^{2^{s-1}}}
$$

for $s>0$. In particular these terms lift to cycles, hence all the further differentials in the spectral sequence map them to zero.

The differential $\bar{\partial}\left(z_{2^{l}(2 m+1)} x_{s_{1}} \cdots x_{s_{n}}\right)$ is given by a sum of the form

$$
\left[\begin{array}{c}
2^{l}(2 m+1) \\
2^{l}
\end{array}\right]_{-1}^{\prime} z_{2^{l+1} m} x_{l} x_{s_{1}} \cdots x_{s_{n}}+\cdots
$$

where the remaining terms start with factors $z_{r}$ with $r<2^{l+1} m$, hence they belong to a higher degree of the filtration with respect to the first term written above.

We note that the coefficient $\left[\begin{array}{c}2^{l}(2 m+1) \\ 2^{l}\end{array}\right]_{-1}^{\prime}$ is non-zero. In particular

$$
\left[\begin{array}{c}
2^{l}(2 m+1) \\
2^{l}
\end{array}\right]_{-1}^{\prime}=\left[\begin{array}{c}
2^{l}(2 m+1) \\
2^{l}
\end{array}\right]_{-1}\left(1-t^{2}\right)^{l-1}
$$

and the coefficient $\left[\begin{array}{c}2^{l}(2 m+1) \\ 2^{l}\end{array}\right]_{-1}$ is invertible, as proved in Lemma 4.3.

Now let $A_{a-1}$ be the module in the column $i$ with $2^{a} \mid i$. A monomial $w$ in $A_{a-1}$ is of the form $w=x_{s_{1}} \cdots x_{s_{n}}$ with $s_{1} \leq \cdots \leq s_{n}$ (of course $n$ may be 0 , that is, $m=1$ ). The monomial $m$ is the image of the element $z_{i} x_{s_{1}} \cdots x_{s_{n}}$ in the $E^{0}$ term of the spectral sequence. By what we have just observed, $z_{i} x_{s_{1}} \cdots x_{s_{n}}$ will survive in the spectral sequence until page $E^{2^{a}}$. If $2^{a+1} \mid i$ then the differential $d^{2^{a}} w$ will be zero. Otherwise, if $i=2^{a}(2 m+1)$, then

$$
d^{2^{a}} w=d^{2^{a}} z_{2^{a}(2 m+1)} x_{s_{1}} \cdots x_{s_{n}}=\left[\begin{array}{c}
2^{a}(2 m+1) \\
2^{a}
\end{array}\right]_{-1}^{\prime} z_{2^{a+1} m} x_{a} x_{s_{1}} \cdots x_{s_{n}},
$$

that is, up to invertible factors,

$$
d^{2^{a}} w=d^{2^{a}} z_{2^{a}(2 m+1)} x_{s_{1}} \cdots x_{s_{n}}=\left(1-t^{2}\right)^{a-1} z_{2^{a+1} m} x_{a} x_{s_{1}} \cdots x_{s_{n}} .
$$

This means that the differential $d^{2^{a}}$ is as described in the statement of the theorem:

$$
d_{i, j}^{k}: z_{i} x \mapsto\left[\begin{array}{c}
i \\
2^{a}
\end{array}\right]_{-1}(1+t)^{2^{a}} z_{i-2^{a} x_{a} x}
$$


The kernel of $d_{i, j}^{k}: A_{a-1} \rightarrow A_{a-1}$ is the ideal of $A_{a-1}$ generated by those monomials that are killed by multiplication by $(1+t)^{2^{a}} x_{a}$, that is, the ideal $\left(x_{0}, \ldots, x_{a}\right)=I_{a}$.

The cokernel of $d_{i, j}^{k}: A_{a-1} \rightarrow A_{a-1}$ is the quotient of $A_{a-1}$ by the ideal generated by $(1+t)^{2^{a}} x_{a}$, that is, $A_{a}$.

Remark 4.6. The proof of Theorem 4.5 gives us a precise description of the generators of the $E^{\infty}$ term:

- The module $I_{h}$ in the $\left[2^{h+1}(2 m+1)\right]$-th column is generated by the terms

$$
\alpha_{2^{h+1}(2 m+1), 0}=\frac{\bar{\partial}\left(z_{2^{h+1}(2 m+1)+1}\right)}{1+t}, \quad \alpha_{2^{h+1}(2 m+1), i}=\frac{\bar{\partial}\left(z_{2^{h+1}(2 m+1)+2^{i}}\right)}{\left(1-t^{2}\right)^{2^{i-1}}}
$$

for $i=1, \ldots, h$, corresponding to the generators $x_{0}, x_{1}, \ldots, x_{h}$ of $I_{h}$; the generator corresponding to the monomial $x_{i} x_{i_{1}} \cdots x_{i_{k}}\left(i_{j} \geq i\right.$ for all $\left.j\right)$ is

$$
\alpha_{2^{h+1}(2 m+1), 0} x_{i_{1}} \cdots x_{i_{k}}=\frac{\bar{\partial}\left(z_{2^{h+1}(2 m+1)+1} x_{i_{1}} \cdots x_{i_{k}}\right)}{1+t}
$$

for $i=0$ and

$$
\alpha_{2^{h+1}(2 m+1), i} x_{i_{1}} \cdots x_{i_{k}}=\frac{\bar{\partial}\left(z_{2^{h+1}(2 m+1)+2^{i}} x_{i_{1}} \cdots x_{i_{k}}\right)}{\left(1-t^{2}\right)^{2 i-1}}
$$

for $i>0$.

- The module $A_{\infty}$ in the 0 -th column is generated by $z_{0}$.

These generators are actually cycles in the algebraic complex $C_{*}(r)$ and naturally lift to generators of the homology $H_{*}\left(\mathrm{~A}_{\mathbf{B}_{r}}, \mathbb{F}_{2}\left[t^{ \pm 1}\right]\right)$ which inherits the structure of an $A\left(\mathbb{F}_{2}\right)\left[t^{ \pm 1}\right]$-module.

Note. When we use the notation

$$
\frac{\bar{\partial} x}{a(t)}
$$

we mean that we consider the boundary of the element $x$ computed in the complex $C_{*}(r)=C_{*}\left(\mathrm{~A}_{\mathbf{B}_{r}}\right)$ with coefficients in the ring of Laurent polynomials over the integers $\mathbb{Z}\left[t^{ \pm 1}\right]$, then we divide exactly by the polynomial $a(t)$ and finally we consider the quotient as a class in the coefficients we are using (for example, $\mathbb{F}_{2}\left[t^{ \pm 1}\right]$ in the case of Remark 4.6).

\subsection{Homology of $\mathrm{B}(2 e, e, r), p=2$}

The result of Theorem 4.5 together with the description of the generators of the modules in the spectral sequence allows us to compute the homology $H_{*}\left(\mathrm{~B}(2 e, e, r), \mathbb{F}_{2}\right)=$ $H_{*}\left(\mathrm{~A}_{\mathbf{B}_{r}}, \mathbb{F}_{2}[t] /\left(1+t^{e}\right)\right)$. We only need to study the long exact homology sequence associated to the short exact sequence

$$
0 \rightarrow \mathbb{F}_{2}\left[t^{ \pm 1}\right] \stackrel{\left(1+t^{e}\right)}{\longrightarrow} \mathbb{F}_{2}\left[t^{ \pm 1}\right] \stackrel{\pi}{\rightarrow} \mathbb{F}_{2}\left[t^{ \pm 1}\right] /\left(1+t^{e}\right) \rightarrow 0
$$

of the coefficients. We can state the following technical result: 
Proposition 4.7. We have a decomposition of the $\mathbb{F}_{2}\left[t^{ \pm 1}\right]$-module

$$
H_{i}\left(\mathrm{~A}_{\mathbf{B}_{r}}, \mathbb{F}_{2}[t] /\left(1+t^{e}\right)\right)=h_{i}(r, e) \oplus h_{i}^{\prime}(r, e)
$$

such that the long exact homology sequence associated to the short exact sequence (3) splits:

$$
0 \rightarrow h_{i+1}^{\prime}(r, e) \stackrel{\delta}{\rightarrow} H_{i}\left(\mathrm{~A}_{\mathbf{B}_{r}}, \mathbb{F}_{2}\left[t^{ \pm 1}\right]\right) \stackrel{\left(1+t^{e}\right)}{\longrightarrow} H_{i}\left(\mathrm{~A}_{\mathbf{B}_{r}}, \mathbb{F}_{2}\left[t^{ \pm 1}\right]\right) \stackrel{\pi_{*}}{\rightarrow} h_{i}(r, e) \rightarrow 0 .
$$

Proof. In order to prove this splitting, for each generator $x$ of the module $H_{i}\left(\mathrm{~A}_{\mathbf{B}_{r}}, \mathbb{F}_{2}[t]\right)$ we provide an element $\tilde{x} \in H_{i+1}\left(\mathrm{~A}_{\mathbf{B}_{r}}, \mathbb{F}_{2}[t] /\left(1+t^{e}\right)\right)$ that maps to $x$ and we prove that $\widetilde{x}$ has right torsion, with respect to the ring $\mathbb{F}_{2}\left[t^{ \pm 1}\right]$, in order to generate a submodule of $H_{i}\left(\mathrm{~A}_{\mathbf{B}_{r}}, \mathbb{F}_{2}[t] /\left(1+t^{e}\right)\right)$ isomorphic to

$$
\operatorname{Ker}\left(\mathbb{F}_{2}\left[t^{ \pm 1}\right] x \stackrel{\left(1+t^{e}\right)}{\longrightarrow} \mathbb{F}_{2}\left[t^{ \pm 1}\right] x\right) .
$$

Let $2^{a}$ be the greatest power of 2 that divides $e$. We observe that the following equivalence holds:

$$
1+t^{e} \equiv 1+t^{2^{a}} \equiv(1+t)^{2^{a}} \bmod 2 .
$$

It turns out that the kernel and the cokernel of the map

$$
\mathbb{F}_{2}\left[t^{ \pm 1}\right] /(1+t)^{2^{i}} \stackrel{\left(1+t^{e}\right)}{\longrightarrow} \mathbb{F}_{2}\left[t^{ \pm 1}\right] /(1+t)^{2^{i}}
$$

are both isomorphic to the quotient

$$
\mathbb{F}_{2}\left[t^{ \pm 1}\right] /\left(1+t^{2^{\min (i, a)}}\right) .
$$

We are going to prove that every direct summand of the $\mathbb{F}_{2}\left[t^{ \pm 1}\right]$-module $H_{i}\left(\mathrm{~A}_{\mathbf{B}_{r}}, \mathbb{F}_{2}[t]\right)$ of the form $\mathbb{F}_{2}\left[t^{ \pm 1}\right] /(1+t)^{2^{i}}$ gives rise to two copies of the module $\mathbb{F}_{2}\left[t^{ \pm 1}\right] /\left(1+t^{\left.2^{\min (i, a)}\right)}\right)$, one in the same dimension, one in one dimension higher. In particular the generator $\alpha_{c, i}$, where $c=2^{h+1}(2 m+1), i \leq h$, determines the two generators

$$
\widetilde{\alpha}_{c, i}=(1+t)^{2^{a}-2^{\min (i, a)}} z_{c+2^{i}}
$$

and

$$
\pi_{*}\left(\frac{1}{(1+t)^{2^{\min (i, a)}}} \delta\left(\widetilde{\alpha}_{c, i}\right)\right)=\pi_{*}\left(\alpha_{c, i}\right)=\frac{\bar{\partial}\left(z_{c+2^{i}}\right)}{(1+t)^{2^{i}}} .
$$

Given a generic monomial $x=\alpha_{c, i} x_{i_{1}} \cdots x_{i_{k}}$ (again $\left.c=(2 m+1) 2^{h+1}, i \leq h\right)$ its projection is given by the cycle

$$
\pi_{*}\left(\alpha_{c, i} x_{i_{1}} \cdots x_{i_{k}}\right)=\frac{\bar{\partial}\left(z_{c+2^{i}} x_{i_{1}} \cdots x_{i_{k}}\right)}{(1+t)^{2^{i}}} .
$$

We remark that, given two elements $x=\alpha_{c, i} x_{j} x_{i_{1}} \cdots x_{i_{k}}$ and $x^{\prime}=\alpha_{c, j} x_{i} x_{i_{1}} \cdots x_{i_{k}}$, since they correspond to the lifting of the same element in the spectral sequence, they represent the same homology class in $H_{*}\left(\mathrm{~A}_{\mathbf{B}_{r}}, \mathbb{F}_{2}\left[t^{ \pm 1}\right]\right)$ (and hence their projections $\pi(x)$ and $\pi\left(x^{\prime}\right)$ are homologous). Hence we can suppose that the monomial $x=\alpha_{c, i} x_{j} x_{i_{1}} \cdots x_{i_{k}}$ is 
written in the form such that $i \leq i_{1} \leq \cdots \leq i_{k}$. We define

$$
\tilde{x}=(1+t)^{2^{a}-2^{\min (i, a)}} z_{c+2^{i}} x_{i_{1}} \cdots x_{i_{k}} .
$$

With this definition it is straightforward to check that $\delta(\tilde{x})$ generates the submodule

$$
\operatorname{Ker}\left(\mathbb{F}_{2}\left[t^{ \pm 1}\right] x \stackrel{\left(1+t^{e}\right)}{\longrightarrow} \mathbb{F}_{2}\left[t^{ \pm 1}\right] x\right)
$$

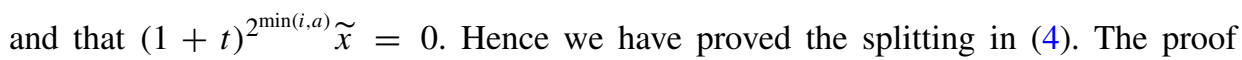
also gives a description of the generators of the homology $H_{i}\left(\mathrm{~A}_{\mathbf{B}_{r}}, \mathbb{F}_{2}[t] /\left(1+t^{e}\right)\right)$ as an $\mathbb{F}_{2}\left[t^{ \pm 1}\right]$-module.

Remark 4.8. As pointed out by the referee, an interesting question would be to get a direct description of the splitting given here and in the proof of Proposition 4.14.

As a consequence we can give a description of the homology of $\mathrm{B}(2 e, e, r)$. Let us define for an integer $n$ the value $h_{p}(n)$ such that $p^{h_{p}(n)} \mid n$ and $p^{h_{p}(n)+1} \nmid n$. For a bigraded module $M$, with degree deg and dimension dim, we use the notation $M[n, m]$ for the module $M^{\prime}$ isomorphic to $M$, but with bigrading shifted such that $\operatorname{deg}^{\prime}=\operatorname{deg}+n, \operatorname{dim}^{\prime}=$ $\operatorname{dim}+m$. Finally, let $M\{n\}=M \oplus M[0,1] \oplus \cdots \oplus M[0, n-1]$

We can state the result as follows:

Theorem 4.9. The sum of the homology groups

$$
\bigoplus_{r \geq 0} H_{*}\left(\mathrm{~B}(2 e, e, r), \mathbb{F}_{2}\right)=\bigoplus_{r \geq 0} H_{*}\left(\mathrm{~A}_{\mathbf{B}_{r}}, \mathbb{F}_{2}[t] /\left(1+t^{e}\right)\right)
$$

is given by the sum

$$
A_{\infty}[1,0] \otimes \mathbb{F}_{2}[t] /\left(1+t^{e}\right)\{2\} \oplus \bigoplus_{n=1}^{\infty} I_{h_{2}(n)}[2 n+1,2 n] \otimes \mathbb{F}_{2}[t] /\left(1+t^{e}\right)\{2\}
$$

We can write explicitly the Poincaré polynomial of the homology $H_{*}\left(\mathrm{~B}(2 e, e, r), \mathbb{F}_{2}\right)$. If we write $P_{2}(\mathrm{~B}(2 e, e, r), u)=\sum_{i=0}^{\infty} \operatorname{dim}_{\mathbb{F}_{2}} H_{i}\left(\mathrm{~B}(2 e, e, r), \mathbb{F}_{2}\right) u^{i}$ for that polynomial, it is more convenient to consider the series in two variables

$$
P_{2}(\mathrm{~B}(2 e, e, *), u, v)=\sum_{r=0}^{\infty} P_{2}(\mathrm{~B}(2 e, e, r), u) v^{r} .
$$

The Poincaré series for the bigraded ring $A_{\infty} /(1+t)^{e}$ is given by

$$
P_{A_{\infty}\left(\mathbb{F}_{2}\right), e}(u, v)=e+\sum_{i=0}^{\infty}\left(2^{\min \left(h_{2}(e), i\right)} u^{2^{i}-1} v^{2^{i}} \prod_{j \geq i} \frac{1}{1-u^{2^{j}-1} v^{2^{j}}}\right)
$$

and the Poincaré series of the ideal $I_{a} \otimes \mathbb{F}_{2}[t] /\left(1+t^{e}\right)$ is given by

$$
P_{I_{a}\left(\mathbb{F}_{2}\right), e}(u, v)=\sum_{i=0}^{a}\left(2^{\min \left(h_{2}(e), i\right)} u^{2^{i}-1} v^{2^{i}} \prod_{j \geq i} \frac{1}{1-u^{2^{j}-1} v^{2^{j}}}\right) .
$$


Hence we obtain the following result:

Corollary 4.10. The Poincaré polynomial of the homology of the groups $\mathrm{B}(2 e, e, r)$ with $\mathbb{F}_{2}$ coefficients is given by

$P_{2}(\mathrm{~B}(2 e, e, *), u, v)=v P_{A_{\infty}\left(\mathbb{F}_{2}\right), e}(u, v)(1+u)+\sum_{n=1}^{\infty} v^{2 n+1} u^{2 n} P_{I_{h_{2}(n)}\left(\mathbb{F}_{2}\right), e}(u, v)(1+u)$.

As an example of these computations we give in Tables 3 and 4 the first homology groups of $\mathrm{B}(2 e, e, r)$ with coefficients in the field $\mathbb{F}_{2}$ and the stable part up to homological dimension 5 .

Table 3. $\operatorname{dim} H_{*}\left(\mathrm{~B}(2 e, e, r), \mathbb{F}_{2}\right), r<8$.

\begin{tabular}{|c|c|c|c|c|c|c|c|c|c|c|}
\hline$r$ & \multicolumn{2}{|c|}{2} & 3 & \multicolumn{3}{|c|}{4} & 5 & \multicolumn{2}{|c|}{6} & 7 \\
\hline$e \bmod (m)$ & $0(2)$ & $1(2)$ & any & $0(4)$ & $2(4)$ & $1(2)$ & any & $0(2)$ & $1(2)$ & any \\
\hline $\operatorname{dim} H_{0}$ & 1 & 1 & 1 & 1 & 1 & 1 & 1 & 1 & 1 & 1 \\
\hline $\operatorname{dim} H_{1}$ & 3 & 2 & 2 & 2 & 2 & 2 & 2 & 2 & 2 & 2 \\
\hline $\operatorname{dim} H_{2}$ & 2 & 1 & 1 & 4 & 4 & 3 & 3 & 3 & 3 & 3 \\
\hline $\operatorname{dim} H_{3}$ & \multicolumn{2}{|c|}{0} & 0 & 7 & 5 & 3 & 3 & 6 & 5 & 5 \\
\hline $\operatorname{dim} H_{4}$ & \multicolumn{2}{|c|}{0} & 0 & 4 & 2 & 1 & 2 & 6 & 4 & 3 \\
\hline $\operatorname{dim} H_{5}$ & \multicolumn{2}{|c|}{0} & 0 & \multicolumn{3}{|c|}{0} & 1 & 5 & 3 & 4 \\
\hline $\operatorname{dim} H_{6}$ & \multicolumn{2}{|c|}{0} & 0 & \multicolumn{3}{|c|}{0} & 0 & 2 & 1 & 3 \\
\hline $\operatorname{dim} H_{7}$ & \multicolumn{2}{|c|}{0} & 0 & \multicolumn{3}{|c|}{0} & 0 & \multicolumn{2}{|c|}{0} & 1 \\
\hline
\end{tabular}

Table 4. $\operatorname{dim} H_{*}\left(\mathrm{~B}(2 e, e, r), \mathbb{F}_{2}\right), r=8$ and stable part up to $H_{5}$.

\begin{tabular}{|c|c|c|c|c|c|}
\hline$r$ & \multicolumn{4}{|c|}{8} & $\geq 9$ \\
\hline$e \bmod (m)$ & $0(8)$ & $4(8)$ & $2(4)$ & $1(2)$ & any \\
\hline $\operatorname{dim} H_{0}$ & 1 & 1 & 1 & 1 & 1 \\
\hline $\operatorname{dim} H_{1}$ & 2 & 2 & 2 & 2 & 2 \\
\hline $\operatorname{dim} H_{2}$ & 3 & 3 & 3 & 3 & 3 \\
\hline $\operatorname{dim} H_{3}$ & 5 & 5 & 5 & 5 & 5 \\
\hline $\operatorname{dim} H_{4}$ & 6 & 6 & 6 & 5 & 5 \\
\hline $\operatorname{dim} H_{5}$ & 8 & 8 & 8 & 6 & 6 \\
\hline $\operatorname{dim} H_{6}$ & 11 & 11 & 9 & 6 & \\
\hline $\operatorname{dim} H_{7}$ & 15 & 11 & 7 & 4 & \\
\hline $\operatorname{dim} H_{8}$ & 8 & 4 & 2 & 1 & \\
\hline
\end{tabular}

Remark 4.11. As pointed out by the referee, our computations here and below give several direct sum decompositions and it would be interesting to make them appear as natural topological decompositions, such as stable decompositions of $X / W$. 
4.5. $H_{*}\left(\mathrm{~A}_{\mathbf{B}_{r}}, \mathbb{F}_{p}\left[t^{ \pm 1}\right]\right)$

As in the case $p=2$ we start by computing the differential in the $E^{2}$ term of the spectral sequence. Again, the starting point is the result in Proposition 4.4. The differential

$$
d_{i, j}^{2}(r): E_{i, j}^{1}(r) \rightarrow E_{i-2, j+1}^{1}(r)
$$

acts as follows:

$$
d_{i, j}^{2}(r): z_{i} x \mapsto\left[\begin{array}{l}
i \\
2
\end{array}\right]_{-1}\left(1-t^{2}\right) z_{i-2} x_{1} x .
$$

The coefficient $\left[\begin{array}{l}i \\ 2\end{array}\right]_{-1}$ is zero for $p \mid i$. Recall in fact that we are considering only even columns, hence even values of $i$. So for $p \mid i$, we have $\varphi_{2 p}(q) \mid\left[\begin{array}{l}i \\ 2\end{array}\right]_{q}$ and when we evaluate the polynomial for $q=-1$ we get $\varphi_{2 p}(-1)=p$.

Hence we can give the picture of the $E^{3}$ page of the spectral sequence: all the odd columns are zero; if $i$ is an even multiple of $p$, then the $i$-th column is isomorphic to the quotient $A\left(\mathbb{F}_{p}\right) /\left((1+t) h,\left(1-t^{2}\right) x_{0}\right)$; if $i+2$ is a multiple of $p$, then the $i$-th column is isomorphic to the submodule of $A\left(\mathbb{F}_{p}\right) /((1+t) h)$ generated by $h$ and $x_{0}$. If $i, i+2$ are not multiples of $p$, then the $i$-th column is isomorphic to the submodule of $A\left(\mathbb{F}_{p}\right) /\left((1+t) h,\left(1-t^{2}\right) x_{0}\right)$ generated by $h$ and $x_{0}$.

As in the case of the prime $p=2$, we need to define some ideals of $A=A\left(\mathbb{F}_{p}\right)$ :

$$
\begin{aligned}
J_{2 a+1}\left(\mathbb{F}_{p}\right) & =J_{2 a+1} \\
& =\left((1+t) h,\left(1-t^{2}\right) x_{0},\left(1-t^{2}\right)^{p-1} y_{1},\left(1-t^{2}\right)^{p} x_{1}, \ldots,\left(1-t^{2}\right)^{p^{a}} x_{a}\right), \\
J_{2 a}\left(\mathbb{F}_{p}\right)= & J_{2 a} \\
= & \left((1+t) h,\left(1-t^{2}\right) x_{0},\left(1-t^{2}\right)^{p-1} y_{1},\left(1-t^{2}\right)^{p} x_{1}, \ldots,\left(1-t^{2}\right)^{(p-1) p^{a-1}} y_{a}\right) .
\end{aligned}
$$

We define the quotients

$$
A_{a}\left(\mathbb{F}_{p}\right)=A_{a}=A\left(\mathbb{F}_{p}\right) / J_{a}\left(\mathbb{F}_{p}\right)
$$

and the ideals

$$
\begin{aligned}
I_{2 a+1}\left(\mathbb{F}_{p}\right) & =I_{2 a+1}=\left(h, x_{0}, y_{1}, x_{1}, \ldots, y_{a}, x_{a}\right) \subset A_{2 a+1}, \\
I_{2 a}\left(\mathbb{F}_{p}\right) & =I_{2 a}=\left(h, x_{0}, y_{1}, x_{1}, \ldots, y_{a}, x_{a}\right) \subset A_{2 a}, \\
K_{2 a}\left(\mathbb{F}_{p}\right) & =K_{2 a}=\left(h, x_{0}, y_{1}, x_{1}, \ldots, x_{a-1}, y_{a}\right) \subset A_{2 a} .
\end{aligned}
$$

Finally, as for $p=2$, we define

$$
J_{\infty}\left(\mathbb{F}_{p}\right)=J_{\infty}=\bigcup_{a=0}^{\infty} J_{a}\left(\mathbb{F}_{p}\right) \quad \text { and } \quad A_{\infty}\left(\mathbb{F}_{p}\right)=A_{\infty}=A / J_{\infty} .
$$

With this notation the $E^{3}$ page of the spectral sequence looks as in Table 5 (at the bottom we give the number of the column).

It turns out that in the following terms the differential until $2 p-2$ is zero, so $E^{3}$ $=E^{2 p-2}$.

Here we have a general description of the spectral sequence in the analogue of Theorem 4.5 for odd primes: 
Table 5. The $E^{3}$ page of the spectral sequence for $p$ odd.

\begin{tabular}{|c|c|c|c|c|c|c|c|c|c}
$A_{1}$ & 0 & $I_{1}$ & $\ldots$ & $I_{1}$ & 0 & $I_{0}$ & 0 & $A_{1}$ & $\ldots$ \\
0 & 1 & 2 & $\ldots$ & $2 p-4$ & $2 p-3$ & $2 p-2$ & $2 p-1$ & $2 p$ & \\
\hline
\end{tabular}

Theorem 4.12. The $k$-th term of the spectral sequence described in Proposition 4.1 computing the homology $H_{*}\left(\mathrm{~A}_{\mathbf{B}_{r}}, \mathbb{F}_{p}\left[t^{ \pm 1}\right]\right)$ is as follows:

- If $k=2 p^{a}$ the $i$-th column is isomorphic to:

- 0 if $i$ is odd;

- I $I_{2 h+1}$ if $2 p^{h} \mid i$ and $2 p^{h+1} \nmid i, 2 p^{h+1} \nmid i+2 p^{h}$ with $h<a$;

$-K_{2 h}$ if $2 p^{h+1} \mid i+2 p^{h}$ with $h<a$;

$-A_{2 a}$ if $2 p^{a} \mid i$.

The differential $d^{2 p^{a}}$ is as follows: if $2 p^{a} \mid i$ and $2 p^{a+1} \nmid i$ we have the map

$$
d_{i, j}^{k}: z_{i} x \mapsto\left[\begin{array}{c}
i \\
2 p^{a}
\end{array}\right]_{-1}\left(1-t^{2}\right)^{p^{a}} z_{i-2 p^{a} x_{a} x}
$$

where the $q$-analog coefficient is invertible; all the other differentials are trivial.

- If $k=2 p^{a}(p-1)$ the $i$-th column is isomorphic to:

- 0 if $i$ is odd;

- I $2 h+1$ if $2 p^{h} \mid i$ and $2 p^{h+1} \nmid i, 2 p^{h+1} \nmid i+2 p^{h}$ with $h \leq a$;

- I ${ }_{2 h}$ if $2 p^{a+1} \mid i+2 p^{a}$

- $K_{2 h}$ if $2 p^{h+1} \mid i+2 p^{h}$ with $h<a$;

$-A_{2 a+1}$ if $2 p^{a+1} \mid i$.

The differential $d^{2 p^{a}(p-1)}$ is as follows: if $2 p^{a+1} \mid i+2 p^{a}$ we have the map

$$
d_{i, j}^{k}: z_{i} x_{a} x \mapsto\left(1-t^{2}\right)^{p^{a}(p-1)} z_{i-2 p^{a}(p-1)} y_{a+1} x
$$

and all the other differentials are trivial.

- If $2 p^{a}<k<2 p^{a}(p-1)$ then $E^{k}=E^{2 p^{a}(p-1)}$ and the differential $d^{k}$ is trivial.

- If $2 p^{a}(p-1)<k<2 p^{a+1}$ then $E^{k}=E^{2 p^{a+1}}$ and the differential $d^{k}$ is trivial.

In the $E^{\infty}$ term of the spectral sequence the $i$-th column is isomorphic to:

- 0 if $i$ is odd;

- $I_{2 h+1}$ if $2 p^{h} \mid i$ and $2 p^{h+1} \nmid i, 2 p^{h+1} \nmid i+2 p^{h}$;

- $K_{2 h}$ if $2 p^{h+1} \mid i+2 p^{h}$;

- $A_{\infty}$ if $i=0$.

The homology $H_{*}\left(\mathrm{~A}_{\mathbf{B}_{r}}, \mathbb{F}_{p}\left[t^{ \pm 1}\right]\right)$ is isomorphic to the graded module associated to the $E^{\infty}$ term. 
Proof. As for the case of $p=2$, we prove the first part of the statement by induction on $a$, and the second part will follow from the first.

We start with the description of $E^{3}$ that we gave in Table 5. In order to work by induction, we will give an explicit description of the generators in the $E^{k}$ term of the spectral sequence.

Let $2 p^{a}<k$ and let $I_{2 h+1}$ be an ideal in the $i$-th column. Hence $2 p^{h} \mid i$ and $2 p^{h+1} \nmid i$, $2 p^{h+1} \nmid i+2 p^{h}$. The generators $h, x_{0}, y_{1}, x_{1}, \ldots, y_{h}, x_{h}$ are the images of the elements $z_{i} h, z_{i} x_{0}, z_{i} y_{1}, z_{i} x_{1}, \ldots, z_{i} y_{h}, z_{i} x_{h}$ in the $E^{0}$ term. We consider these generators of the ideal $I_{2 h+1}$ ordered as we wrote them, so $h$ will be the smallest generator and $x_{h}$ will be the biggest. Let $m$ be a generic monomial in the ideal $I_{2 h+1}$. We write its factors ordered from the smallest to the biggest. It is the image of the element $z_{i} m$ in the $E^{0}$ term of the spectral sequence. Its lifting in the $E^{k}$ term is

$$
\frac{\bar{\partial}\left(z_{i+1} m^{\prime}\right)}{1+t}
$$

if the smallest factor of $m$ is $h$ and $m=h m^{\prime}$, and

$$
\frac{\bar{\partial}\left(z_{i+2 p^{s}} m^{\prime}\right)}{\left(1-t^{2}\right)^{p^{s}}}
$$

if the smallest factor of $m$ is $x_{s}$ and $m=x_{s} m^{\prime}$. Note that we must have $s \leq h$ and hence $2 p^{h+1} \nmid i+2 p^{s}$. This implies that the coefficient of $z_{i} x_{s} m^{\prime}$ in $\bar{\partial}\left(z_{i+2} p^{s} m^{\prime}\right)$ is non-zero.

If the smallest factor of $m$ is $y_{s}$ and we let $m=y_{s} m^{\prime}$, we need to define the following element. Let $\bar{\partial}[p]\left(z_{i+2 p^{s}} m^{\prime}\right)$ be the sum of all terms that appear in $\bar{\partial}\left(z_{i+2 p^{s}} m^{\prime}\right)$ with a coefficient that is divisible by $p$ (when we consider the boundary with integer coefficients). Notice that, with respect to the filtration $\mathcal{F}$ of the complex, the highest term that does not appear in the sum is $\left(1-t^{2}\right)^{p^{s}} z_{i} x_{s} m^{\prime}$. We define

$$
\gamma[p]\left(z_{i+2 p^{s}} m^{\prime}\right)=\frac{\bar{\partial}[p]\left(z_{\left.i+2 p^{s}\right) m^{\prime}}\right.}{p\left(1-t^{2}\right)^{p^{s-1}}}
$$

and we find that the lifting of $z_{i} m$ is

$$
\frac{\bar{\partial}\left(\gamma[p]\left(z_{i+2} p^{s} m^{\prime}\right)\right)}{\left(1-t^{2}\right)^{(p-1) p^{s-1}}} .
$$

In fact it is clear that the difference

$$
\frac{\gamma[p]\left(z_{i}+2 p^{s} m^{\prime}\right)}{\left(1-t^{2}\right)^{(p-1) p^{s-1}}}-z_{i} y_{s} m^{\prime}
$$

projects to a boundary in the quotient $\mathcal{F}_{i+1} C_{*}(r) / \mathcal{F}_{i} C_{*}(r)$. Notice that the quotient

$$
\frac{\gamma[p]\left(z_{i+2 p^{s}} m^{\prime}\right)}{\left(1-t^{2}\right)^{(p-1) p^{s-1}}}
$$


is not defined in an $\mathbb{F}_{p}\left[t^{ \pm 1}\right]$-module but still we can define it if we consider only the summands of $\gamma[p]\left(z_{i+2 p^{s}} m^{\prime}\right)$ that are not in $\mathcal{F}_{i} C_{*}(r)$. Hence we can use the first term of the difference, instead of the second, to lift the class of $z_{i} m$ to a representative in $E^{k}$.

Since all the liftings that we have defined are global cycles, all the following differentials in the spectral sequence map these terms to zero.

We now give a description of the generators of the ideal $I_{2 h}$ appearing in the $i$-th column of the $E^{k}$ term of the spectral sequence. We must have $2 p^{a+1} \mid i+2 p^{a}$. As before, the ideal $I_{2 h}$ is generated by the terms $h, x_{0}, y_{1}, x_{1}, \ldots, y_{h}, x_{h}$ that are the images of the elements $z_{i} h, z_{i} x_{0}, z_{i} y_{1}, z_{i} x_{1}, \ldots, z_{i} y_{h}, z_{i} x_{h}$ in the $E^{0}$ term.

Given a monomial $m$ with smallest factor different from $x_{h}$, it is easy to verify that the lifting is the same as in the previous description of the ideal $I_{2 h+1}$.

Let now $m$ have smallest term $x_{h}$, with $m=x_{h} m^{\prime}$. Let $d=h+d^{\prime}$ be such that $2 p^{d} \mid i+2 p^{h}$ and $2 p^{d+1} \nmid i+2 p^{h}$. One can verify that $\left(1-t^{2}\right)^{p^{h}} z_{i} x_{h} m^{\prime}$ is the first non-zero element in

$$
\frac{\bar{\partial}\left[p^{d^{\prime}}\right]\left(z_{i+2 p^{h}}\right) m^{\prime}}{p^{d^{\prime}}}
$$

since all the previous summands in $\bar{\partial}\left[p^{d^{\prime}}\right]\left(z_{i+2 p^{h}}\right) m^{\prime}$ (when we consider the boundary with integer coefficients) have a coefficient divisible by $p^{d^{\prime}+1}$. The first term missing in the sum $\bar{\partial}\left[p^{d^{\prime}}\right]\left(z_{i+2 p^{h}}\right) m^{\prime}$ is the monomial $\left(1-t^{2}\right)^{p^{h+1}} z_{i-(p-1)} p^{h} x_{h+1} m^{\prime}$. It follows that we can take as a lifting of $x_{h} m^{\prime}$ in $E^{2 p^{h}(p-1)}$ the element

$$
\frac{\bar{\partial}\left[p^{d^{\prime}}\right]\left(z_{\left.i+2 p^{h}\right) m^{\prime}}\right.}{p^{d^{\prime}}\left(1-t^{2}\right) p^{h}}
$$

and hence its differential with respect to the map $d^{2 p^{h}(p-1)}$ is

$$
\bar{\partial} \frac{\bar{\partial}\left[p^{d^{\prime}}\right]\left(z_{i+2 p^{h}}\right) m^{\prime}}{p^{d^{\prime}}\left(1-t^{2}\right) p^{h}}
$$

that is homologous, by what we have seen before, to the element

$$
\left(1-t^{2}\right)^{p^{h}(p-1)} z_{i-(p-1)} p^{h} y_{h+1} m^{\prime}
$$

as stated in the theorem.

Hence the differential $d^{2 p^{h}(p-1)}$ maps $x_{h} m^{\prime} \mapsto\left(1-t^{2}\right)^{p^{h}(p-1)} y_{h+1} m^{\prime}$ and is zero for all the other elements. Clearly the kernel is the ideal $K_{2 h}$.

For what concerns the ideal $K_{2 h}$, generated by $h, x_{0}, y_{1}, x_{1}, \ldots, y_{h}$, the description of the generators given before can be repeated and all the generators lift to global cycles as soon as $k>2 p^{h}(p-1)$.

Finally we consider the modules $A_{2 h}$ and $A_{2 h+1}$ that appear in column $i$ of the spectral sequence. Each monomial $m$ of $A_{2 h}$ or $A_{2 h+1}$ corresponds to a monomial $z_{i} m$ in the $E^{0}$ term of the spectral sequence. If $2 p^{a} \mid i$ then the monomial $z_{i} m$ will survive until the 
$E^{2 p^{a}}$ term of the spectral sequence, since all the first summands of the differential $\bar{\partial} z_{i} m$ are zero. The first summand that can be non-zero is

$$
d_{i, j}^{k}: z_{i} x \mapsto\left[\begin{array}{c}
i \\
2 p^{a}
\end{array}\right]_{-1}\left(1-t^{2}\right)^{p^{a}} z_{i-2 p^{a}} x_{a} x,
$$

which is actually non-zero if and only if $2 p^{a} \mid i$ and $2 p^{a+1} \nmid i$. The kernel of the map $d^{2 p^{a}}: A_{2 a} \rightarrow A_{2 a}$ is the ideal $I_{2 a}$ and the quotient of the kernel by the image of $d^{2 p^{a}}$, when the image is non-zero, is the module $A_{2 a+1}$. Hence all the other differentials are forced to be zero and the behaviour of the spectral sequence is as described in the theorem.

Remark 4.13. From the proof we can read off the description of the generators of the $E^{\infty}$ term:

- The module $I_{2 h+1}$ in the $2 p^{h} n$-th column with $n \not \equiv 0,-1 \bmod p$ is generated by the terms

$$
\begin{aligned}
\beta_{2 p^{h} n, h} & =\frac{\bar{\partial}\left(z_{2 p^{h} n+1}\right)}{1+t}, \\
\beta_{2 p^{h} n, x_{i}} & =\frac{\bar{\partial}\left(z_{\left.2 p^{h} n+2 p^{i}\right)}\right.}{\left(1-t^{2}\right)^{i}}, \\
\beta_{2 p^{h} n, y_{i}} & =\frac{\bar{\partial}\left(\gamma_{2 p^{h} n, y_{i}}\right)}{\left(1-t^{2}\right)^{(p-1) p^{i-1}}}
\end{aligned}
$$

where we set

$$
\gamma_{2 p^{h} n, y_{i}}=\frac{\bar{\partial}\left(z_{2 p^{h} n+2 p^{i}}\right)-\sum_{2 p^{h} n, y_{i}}(p)}{p\left(1-t^{2}\right)^{p^{i-1}}},
$$

and $\sum_{2 p^{h} n, y_{i}}(p)$ is the sum of the terms in $\bar{\partial}\left(z_{2 p^{h} n+2 p^{i}}\right)$ that have a coefficient not divisible by $p$. Notice that the first of these terms is $\left(1-t^{2}\right)^{p^{i}} z_{2 p^{h} n} x_{i}$.

- The module $K_{2 h}$ in the $2 p^{h}(p n-1)$-th column is generated by the terms

$$
\begin{aligned}
\beta_{2 p^{h}(p n-1), h} & =\frac{\bar{\partial}\left(z_{2 p^{h}(p n-1)+1}\right)}{(1+t)}, \\
\beta_{2 p^{h}(p n-1), x_{i}} & =\frac{\bar{\partial}\left(z_{\left.2 p^{h}(p n-1)+2 p^{i}\right)}\right.}{\left(1-t^{2}\right)^{i}}, \\
\beta_{2 p^{h}(p n-1), y_{i}} & =\frac{\bar{\partial}\left(\gamma_{2 p^{h}(p n-1), y_{i}}\right)}{\left(1-t^{2}\right)^{(p-1) p^{i-1}}}
\end{aligned}
$$

with, for $i<h$,

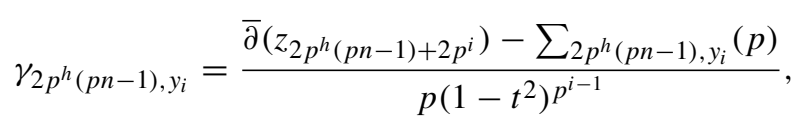


while for $i=h$ we set

$$
\gamma_{2 p^{h}(p n-1), y_{h}}=\frac{\bar{\partial}\left(z_{2 p^{h+1} n}\right)-\sum_{2 p^{h}(p n-1), y_{h}}\left(p^{2}\right)}{p^{2}\left(1-t^{2}\right)^{p^{h-1}}}
$$

where $\sum_{2 p^{h}(p n-1), y_{h}}\left(p^{2}\right)$ is the sum of the terms in $\bar{\partial}\left(z_{2 p^{h+1} n}\right)$ that have coefficients not divisible by $p^{2}$. Notice that the first of these terms is $p\left(1-t^{2}\right)^{p^{h}} z_{2 p^{h}(p n-1)} x_{h}$.

- The module $A_{\infty}$ in the 0 -th column is generated by $z_{0}$.

\subsection{Homology of $\mathrm{B}(2 e, e, r), p>2$}

Now we compute $H_{*}\left(\mathrm{~B}(2 e, e, r), \mathbb{F}_{p}\right)=H_{*}\left(\mathrm{~A}_{\mathbf{B}_{r}}, \mathbb{F}_{p}[t] /\left(1-(-t)^{e}\right)\right)$ by means of the long exact homology sequence associated to the short exact sequence

$$
0 \rightarrow \mathbb{F}_{p}\left[t^{ \pm 1}\right] \stackrel{\left(1-(-t)^{e}\right)}{\longrightarrow} \mathbb{F}_{p}\left[t^{ \pm 1}\right] \stackrel{\pi}{\rightarrow} \mathbb{F}_{p}\left[t^{ \pm 1}\right] /\left(1-(-t)^{e}\right) \rightarrow 0 .
$$

As in the previous section, we have a splitting result:

Proposition 4.14. We have a decomposition of the $\mathbb{F}_{p}\left[t^{ \pm 1}\right]$-module

$$
H_{i}\left(\mathrm{~A}_{\mathbf{B}_{r}}, \mathbb{F}_{p}[t] /\left(1-(-t)^{e}\right)\right)=h_{i}(r, e) \oplus h_{i}^{\prime}(r, e)
$$

such that the long exact homology sequence associated to the short exact sequence in (3) splits:

$$
0 \rightarrow h_{i+1}^{\prime}(r, e) \stackrel{\delta}{\rightarrow} H_{i}\left(\mathrm{~A}_{\mathbf{B}_{r}}, \mathbb{F}_{p}\left[t^{ \pm 1}\right]\right) \stackrel{\left(1+t^{e}\right)}{\longrightarrow} H_{i}\left(\mathrm{~A}_{\mathbf{B}_{r}}, \mathbb{F}_{p}\left[t^{ \pm 1}\right]\right) \stackrel{\pi_{*}}{\rightarrow} h_{i}(r, e) \rightarrow 0 .
$$

Proof. We start by observing that, since $1+t$ and $1-t$ are co-prime in $\mathbb{F}_{p}\left[t^{ \pm 1}\right]$, we can split any module of the form $\mathbb{F}_{p}\left[t^{ \pm 1}\right] /\left(1-t^{2}\right)^{l}$ as a direct sum

$$
\mathbb{F}_{p}\left[t^{ \pm 1}\right] /(1+t)^{l} \oplus \mathbb{F}_{p}\left[t^{ \pm 1}\right] /(1-t)^{l}
$$

of two modules, generated respectively by $(1-t)^{l}$ and $(1+t)^{l}$.

Moreover we consider the following properties of $1-(-t)^{e}$ in $\mathbb{F}_{p}\left[t^{ \pm 1}\right]$ :

$$
\left(1-t, 1-(-t)^{e}\right)= \begin{cases}(1-t) & \text { if } e \text { is even, } \\ (1) & \text { if } e \text { is odd, }\end{cases}
$$

and

$$
\left(1+t, 1-(-t)^{e}\right)= \begin{cases}(1+t) & \text { if } e \text { is even or if } p \nmid e, \\ (1+t)^{p^{i}} & \text { if } e \text { is odd and } p^{i} \mid e, \text { but } p^{i+1} \nmid e .\end{cases}
$$

The second equality follows from the fact that the polynomial $1+t$ divides the cyclotomic polynomial $\varphi_{2 p^{i}}$ with order exactly $\phi\left(p^{i}\right)=(p-1) p^{i-1}$ (in $\mathbb{F}_{p}\left[t^{ \pm 1}\right]$ ) and is co-prime to all the other cyclotomic polynomials.

Now, let us fix the value of $e$. When we study the exact sequence (6) we need to consider only the highest power of $p$ that divides $e$, and whether $e$ is even or odd. 
Let us consider the monomial $x=z_{2 m} h^{r} y_{i_{1}}^{s_{i_{1}}} \cdots y_{i_{n}}^{s_{i_{n}}} x_{k_{1}} \cdots x_{k_{m}}$ in $H_{i}\left(\mathrm{~A}_{\mathbf{B}_{r}}, \mathbb{F}_{p}\left[t^{ \pm 1}\right]\right)$. We suppose that the indices are ordered so that $i_{1}<\cdots<i_{n}$ and $k_{1}<\cdots<k_{m}$. We want to define a lifting $\tilde{x}$ of $x$ in $H_{i}\left(\mathrm{~A}_{\mathbf{B}_{r}}, \mathbb{F}_{p}[t] /\left(1-(-t)^{e}\right)\right)$. To do this we have to consider different cases.

First consider the case of $e$ even. We define

$$
\tilde{x}= \begin{cases}\frac{1-(-t)^{e}}{1+t} z_{2 m+1} h^{r-1} y_{i_{1}}^{s_{i_{1}}} \cdots y_{i_{n}}^{s_{i_{n}}} x_{k_{1}} \cdots x_{k_{m}} & \text { if } r>0, \\ \frac{1-(-t)^{e}}{1-t^{2}} z_{2 m+2 p^{i_{0}} y_{i_{1}}^{s_{i_{1}}} \cdots y_{i_{n}}^{s_{i_{n}}} x_{k_{2}} \cdots x_{k_{m}}} & \text { if } r=0 \text { and } k_{1}<i_{1}, \\ \frac{1-(-t)^{e}}{1-t^{2}} \gamma_{2 m, y_{i_{1}}} y_{i_{1}}^{s_{i_{1}}-1} y_{i_{2}}^{s_{i_{2}}} \cdots y_{i_{n}}^{s_{i_{n}}} x_{k_{2}} \cdots x_{k_{m}} & \text { if } i_{1} \leq k_{1} .\end{cases}
$$

Now we suppose $e$ is odd and let $j$ be an integer such that $p^{j} \mid e$ and $p^{j+1} \nmid e$. Then set again

$$
\tilde{x}= \begin{cases}\frac{1-(-t)^{e}}{1+t} z_{2 m+1} h^{r-1} y_{i_{1}}^{s_{i_{1}}} \cdots y_{i_{n}}^{s_{i_{n}}} x_{k_{1}} \cdots x_{k_{m}} & \text { if } r>0, \\ \frac{1-(-t)^{e}}{(1+t)^{\min \left(j, i_{0}\right)}} z_{2 m+2 p^{i_{1}}} y_{i_{1}}^{s_{i_{1}}} \cdots y_{i_{n}}^{s_{i_{n}}} x_{k_{2}} \cdots x_{k_{m}} & \text { if } r=0 \text { and } k_{1}<i_{1}, \\ \frac{1-(-t)^{e}}{(1+t)^{\min \left(p^{j},(p-1) p^{i_{1}-1}\right)}} \gamma_{2 m, y_{i_{1}}} y_{i_{1}}^{s_{i_{1}}-1} y_{i_{2}}^{s_{i_{2}}} \cdots y_{i_{n}}^{s_{i_{n}}} x_{k_{2}} \cdots x_{k_{m}} & \text { if } i_{1} \leq k_{1} .\end{cases}
$$

It is clear from the definitions that $\delta(\tilde{x})=x$ and that the $\mathbb{F}_{p}\left[t^{ \pm 1}\right]$-module generated by $\tilde{x}$ is isomorphic to the submodule

$$
\operatorname{Ker}\left(\mathbb{F}_{p}\left[t^{ \pm 1}\right] x \stackrel{\left(1-(-t)^{e}\right)}{\longrightarrow} \mathbb{F}_{p}\left[t^{ \pm 1}\right] x\right)
$$

of $H_{i}\left(\mathrm{~A}_{\mathbf{B}_{r}}, \mathbb{F}_{p}\left[t^{ \pm 1}\right]\right)$. Hence the same argument of Proposition 4.7 holds: the map

$$
\delta: H_{i}\left(\mathrm{~A}_{\mathbf{B}_{r}}, \mathbb{F}_{p}[t] /\left(1-(-t)^{e}\right)\right) \rightarrow \operatorname{Ker}\left(H_{i}\left(\mathrm{~A}_{\mathbf{B}_{r}}, \mathbb{F}_{p}\left[t^{ \pm 1}\right]\right) \stackrel{\left(1-(-t)^{e}\right)}{\longrightarrow} H_{i}\left(\mathrm{~A}_{\mathbf{B}_{r}}, \mathbb{F}_{p}\left[t^{ \pm 1}\right]\right)\right)
$$

has a section and the statement of the proposition holds, with $h_{*}(r, e)$ generated by all elements of type $\pi_{*}(x)$ and $h_{*}^{\prime}(r, e)$ generated by all elements of type $\tilde{x}$.

We can state the result for the $\mathbb{F}_{p}$-homology of $\mathrm{B}(2 e, e, r)$ as follows:

Theorem 4.15. Let $p$ be an odd prime. Then

$$
H_{*}\left(\mathrm{~A}_{\mathbf{B}_{*}}, \mathbb{F}_{p}[t] /\left(1-(-t)^{e}\right)\right)=\left(M_{1} \oplus M_{2} \oplus M_{3}\right) \otimes \mathbb{F}_{p}[t] /\left(1-(-t)^{e}\right)\{2\}
$$

where

$$
\begin{aligned}
& M_{1}=A_{\infty}[1,0], \\
& M_{2}=\bigoplus_{n \geq 1} K_{2 h_{p}(n)}\left[2\left(p n-p^{h_{p}(n)}\right)+1,2\left(p n-p^{h_{p}(n)}\right)\right], \\
& M_{3}=\bigoplus_{n \geq 1} I_{2 h_{p}(n)+1}[2 n+1,2 n]
\end{aligned}
$$


and the direct sum in $M_{3}$ is over $n$ such that $h_{p}(n)=h_{p}\left(n+p^{h_{p}(n)}\right)$, that is, $n$ is not of the form $p^{h-1} s$ with $p \nmid s$.

We can now give the Poincaré polynomial of $H_{*}\left(\mathrm{~B}(2 e, e, r), \mathbb{F}_{p}\right)$, denoted by $P_{p}(\mathrm{~B}(2 e, e, r), u)=\sum_{i=0}^{\infty} \operatorname{dim}_{\mathbb{F}_{p}} H_{i}\left(\mathrm{~B}(2 e, e, r), \mathbb{F}_{p}\right) u^{i}$. We can consider the series in two variables

$$
P_{p}(\mathrm{~B}(2 e, e, *), u, v)=\sum_{r=0}^{\infty} P_{p}(\mathrm{~B}(2 e, e, r), u) v^{r} .
$$

The Poincaré series for the bigraded ring $A_{\infty} /\left(1-(-t)^{e}\right)$ is given by

$$
P_{A_{\infty}\left(\mathbb{F}_{p}\right), e}(u, v)= \begin{cases}\frac{1}{1-v} \prod_{i \geq 1} \frac{1}{1-u^{2 p^{i}-2} v^{2 p^{i}}} \prod_{j \geq 0}\left(1+u^{2 p^{j}-1} v^{2 p^{j}}\right) & \text { for } e \text { odd } \\ \prod_{0}^{p}+\sum_{0}^{\infty}(e)+\sum_{0}^{p}(e) & \text { for } e \text { even }\end{cases}
$$

where we define the following terms:

$$
\begin{aligned}
\prod_{0}^{p} & =v \frac{1}{1-v} \prod_{i \geq 1} \frac{1}{1-u^{2 p^{i}-2} v^{2} p^{i}} \prod_{j \geq 0}\left(1+u^{2 p^{j}-1} v^{2 p^{j}}\right), \\
\sum_{k_{1}}^{k_{2}}(e) & =\sum_{r=k_{1}}^{k_{2}} 2 p^{\min \left(h_{p}(e), r\right)} u^{2 p^{r}-1} v^{2 p^{r}} \prod_{i \geq r+1} \frac{1}{1-u^{2 p^{i}-2} v^{2 p^{i}}} \prod_{j \geq r}\left(1+u^{2 p^{j}-1} v^{2 p^{j}}\right), \\
\sum_{k_{1}}^{k_{2}}{ }_{2}^{p}(e) & =\sum_{r=k_{1}}^{k_{2}} 2 \min \left(p^{h_{p}(e)},(p-1) p^{r-1}\right) u^{2 p^{r}-2} v^{2 p^{r}} \\
& \times \prod_{i \geq r} \frac{1}{1-u^{2 p^{i}-2} v^{2 p^{i}}} \prod_{j \geq r}\left(1+u^{2 p^{j}-1} v^{2 p^{j}}\right) .
\end{aligned}
$$

The Poincaré series of the ideal $I_{2 a+1} \otimes \mathbb{F}_{p}[t] /\left(1+t^{e}\right)$ is given by

$$
P_{I_{2 a+1}\left(\mathbb{F}_{p}\right), e}(u, v)= \begin{cases}\prod_{0}^{p}+\sum_{0}^{a}(1)+\sum_{1}^{a}(1) & \text { for } e \text { odd }, \\ \prod_{0}^{p}+\sum_{0}^{a}{ }_{1}^{p}(e)+\sum_{1}^{a}{ }_{2}^{p}(e) & \text { for } e \text { even } .\end{cases}
$$

The Poincaré series of the ideal $K_{2 a} \otimes \mathbb{F}_{p}[t] /\left(1+t^{e}\right)$ is given by

$$
P_{K_{2 a}\left(\mathbb{F}_{p}\right), e}(u, v)=\left\{\begin{array}{l}
\prod_{0}^{p}+\sum_{0}^{a-1} p(1)+\sum_{1}^{a} p(1) \text { for } e \text { odd } \\
\prod_{0}^{p}+\sum_{0}^{a-1} p(e)+\sum_{1}^{a}{ }_{2}^{p}(e) \text { for } e \text { even }
\end{array}\right.
$$

Hence we obtain: 
Corollary 4.16. Let $p$ be an odd prime. The Poincaré polynomial of the homology of the groups $\mathrm{B}(2 e, e, r)$ with $\mathbb{F}_{p}$ coefficients is

$$
P_{p}(\mathrm{~B}(2 e, e, *), u, v)=(1+v)(A+B+C)
$$

where

$$
\begin{aligned}
A & =v P_{A_{\infty}\left(\mathbb{F}_{p}\right), e}(u, v), \\
B & =\sum_{n \geq 1} v^{2\left(p n-p^{h_{p}(n)}\right)+1} u^{2\left(p n-p^{h_{p}(n)}\right)} P_{K_{2 h_{p}(n)}\left(\mathbb{F}_{p}\right), e}(u, v), \\
C & =\sum_{n \geq 1} v^{2 n+1} u^{2 n} P_{I_{2 h_{p}(n)+1}\left(\mathbb{F}_{p}\right), e}(u, v),
\end{aligned}
$$

and the sum in $C$ is over $n$ such that $h_{p}(n)=h_{p}\left(n+p^{h_{p}(n)}\right)$, that is, $n$ is not of the form $p^{h-1} s$ with $p \nmid s$.

As an example of these computations we give in Table 6 the first homology groups of $\mathrm{B}(2 e, e, r)$ with coefficients in the field $\mathbb{F}_{3}$ and the stable part up to homological dimension 5 . For a prime $p \geq 5$ there is no $p$-torsion in the integral homology $H_{*}(\mathrm{~B}(2 e, e, r), \mathbb{Z})$ for $r \leq 8$. Actually, for $p$ an odd prime, the first $p$-torsion in $H_{*}(\mathrm{~B}(2 e, e, r), \mathbb{Z})$ appears for $r=2 p$, as it comes from the classes associated to the generators $x_{1}, y_{1}$ in $H_{*}\left(\mathrm{~B}(2 e, e, r), \mathbb{F}_{p}\right)$.

Table 6. $\operatorname{dim} H_{*}\left(\mathrm{~B}(2 e, e, r), \mathbb{F}_{3}\right), r<8$.

\begin{tabular}{|c|c|c|c|c|c|c|c|c|c|c|c|c|c|c|}
\hline$r$ & \multicolumn{2}{|c|}{2} & 3 & \multicolumn{2}{|c|}{4} & 5 & \multicolumn{3}{c|}{6} & 7 & \multicolumn{2}{|c|}{8} & $\geq 9$ \\
\hline$e \bmod (m)$ & $0(2)$ & $1(2)$ & any & $0(2)$ & $1(2)$ & any & $0(6)$ & $2,4(6)$ & $1(2)$ & any & $0(2)$ & $1(2)$ & any \\
\hline $\operatorname{dim} H_{0}$ & 1 & 1 & 1 & 1 & 1 & 1 & 1 & 1 & 1 & 1 & 1 & 1 & 1 \\
\hline $\operatorname{dim} H_{1}$ & 3 & 2 & 2 & 2 & 2 & 2 & 2 & 2 & 2 & 2 & 2 & 2 & 2 \\
\hline $\operatorname{dim} H_{2}$ & 2 & 1 & 2 & 2 & 2 & 2 & 2 & 2 & 2 & 2 & 2 & 2 & 2 \\
\hline $\operatorname{dim} H_{3}$ & 0 & 1 & 3 & 2 & 2 & 2 & 2 & 2 & 2 & 2 & 2 & 2 \\
\hline $\operatorname{dim} H_{4}$ & 0 & 0 & 2 & 1 & 2 & 6 & 4 & 3 & 3 & 3 & 3 & 3 \\
\hline $\operatorname{dim} H_{5}$ & 0 & 0 & 0 & 1 & 11 & 7 & 4 & 4 & 6 & 5 & 5 \\
\hline $\operatorname{dim} H_{6}$ & 0 & 0 & 0 & 0 & 6 & 4 & 2 & 2 & 7 & 5 & \\
\hline $\operatorname{dim} H_{7}$ & 0 & 0 & 0 & 0 & & 0 & & 1 & 5 & 3 & \\
\hline
\end{tabular}

\subsection{An example of the computation of torsion in integral homology}

We are not able to compute the whole integral homology of the groups $\mathrm{B}(2 e, e, r)$, but we provide a simple Bockstein computation in order to complete the proof of Theorem 1.4.

According to the notation in the proof of Proposition 4.7, the homology module $\mathrm{H}_{2}\left(\mathrm{~B}(2 e, e, r+4), \mathbb{F}_{2}\right)$ is generated by the cycles

$$
\pi_{*}\left(z_{2} x_{0}^{2+r}\right), \quad \widetilde{x_{0}^{2+r} x_{1}}, \quad \pi_{*}\left(x_{0}^{r} x_{1}^{2}\right) .
$$


It is straightforward to check that the classes in $H_{2}(\mathrm{~B}(2 e, e, r+4), \mathbb{Z})$ corresponding to $\pi_{*}\left(z_{2} x_{0}^{2+r}\right)$ and $\widetilde{x_{0}^{2+r} x_{1}}$ project to generators of $H_{2}(\mathrm{~B}(2 e, e, r+4), \mathbb{Q})$, hence they generate torsion-free $\mathbb{Z}$-modules.

The generator $\pi_{*}\left(x_{0}^{r} x_{1}^{2}\right)$ is the image of an element $\rho \in H_{2}(\mathrm{~B}(2 e, e, r+4), \mathbb{Z})$. We claim that $\rho$ has 2 -torsion. In order to prove this we will use the Bockstein exact sequence associated to the extension

$$
0 \rightarrow \mathbb{Z}_{2} \stackrel{2}{\rightarrow} \mathbb{Z}_{4} \rightarrow \mathbb{Z}_{2} \rightarrow 0
$$

In particular, we show that the Bockstein differential $\beta_{2}$ maps $\pi_{*}\left(x_{0}^{r} x_{2}\right) \mapsto \pi_{*}\left(x_{0}^{2} x_{1}^{2}\right)$. It follows by a standard argument that $\pi_{*}\left(x_{0}^{2} x_{1}^{2}\right)$ generates a $\mathbb{Z}_{2}$-torsion class in $H_{2}\left(\mathrm{~B}(2 e, e, r+4), \mathbb{Z}_{4}\right)$ and hence, by the Universal Coefficients Theorem, in $\mathrm{H}_{2}(\mathrm{~B}(2 e, e, r+4), \mathbb{Z})$.

In order to compute $\beta_{2}\left(\pi_{*}\left(x_{0}^{r} x_{2}\right)\right)$ recall that the class of the element $\pi_{*}\left(x_{0}^{r} x_{1}^{2}\right)$ in $\mathrm{H}_{2}\left(\mathrm{~B}(2 e, e, r+4), \mathbb{F}_{2}\right)$ is

$$
\begin{cases}\frac{\bar{\partial}\left(z_{2} x_{1}\right)}{1-t^{2}} & \text { for } r=0, \\ \frac{\bar{\partial}\left(z_{1} x_{0}^{r-1} x_{1}^{2}\right)}{1+t} & \text { for } r>0,\end{cases}
$$

and the class of $\pi_{*}\left(x_{0}^{r} x_{2}\right)$ in $H_{3}\left(\mathrm{~B}(2 e, e, r+4), \mathbb{F}_{2}\right)$ is

$$
\begin{cases}\frac{\bar{\partial}\left(z_{4}\right)}{\left(1-t^{2}\right)^{2}} & \text { for } r=0, \\ \frac{\bar{\partial}\left(z_{1} x_{0}^{r-1} x_{2}\right)}{1+t} & \text { for } r>0 .\end{cases}
$$

Their liftings to the complex with $\mathbb{Z}_{4}$ coefficients are

$$
\frac{\bar{\partial}\left(z_{4}\right)-2 z_{2} x_{1}}{\left(1-t^{2}\right)^{2}} \text { and } \frac{\bar{\partial}\left(z_{1} x_{0}^{r-1} x_{2}\right)-2 z_{1} x_{0}^{r-1} x_{1}^{2}}{1+t},
$$

respectively. Now the claim about the Bockstein map follows since it is clear that the differential calculated for the chosen liftings gives exactly the double of the cycle $\pi_{*}\left(x_{0}^{r} x_{1}^{2}\right)$.

Our next purpose is to show that in general there can be $p^{2}$-torsion in the integral homology of $\mathrm{B}(2 e, e, r)$. We will actually prove that there is a class of 4-torsion in $H_{7}(\mathrm{~B}(16,8,8), \mathbb{Z})$.

We consider the homology class $\tilde{x}_{3} \in H_{8}\left(\mathrm{~B}(16,8,8), \mathbb{F}_{2}\right)$ that is represented by $\pi_{*}\left(z_{8}\right)$. It generates an $\mathbb{F}_{2}\left[t^{ \pm 1}\right]$-module isomorphic to $\mathbb{F}_{2}\left[t^{ \pm 1}\right] /\left(1-t^{2}\right)^{4}$. We want to compute the Bockstein $\beta_{2}$ of $\tilde{x}_{3}$.

With the description given in Section 4.1 we can compute, with coefficients in $\mathbb{Z}\left[t^{ \pm 1}\right]$,

$$
\begin{aligned}
\bar{\partial} z_{8}=\bar{\partial}\left(\overline{1} 1^{7}\right)= & {\left[\begin{array}{l}
8 \\
2
\end{array}\right]_{-1}\left(1-t^{2}\right) \overline{1} 1^{5} 01+\left[\begin{array}{l}
8 \\
4
\end{array}\right]_{-1}\left(1-t^{2}\right)^{2} \overline{1} 1^{3} 01^{3} } \\
& +\left[\begin{array}{l}
8 \\
6
\end{array}\right]_{-1}\left(1-t^{2}\right)^{3} \overline{1} 101^{5}+\left[\begin{array}{l}
8 \\
8
\end{array}\right]_{-1}\left(1-t^{2}\right)^{4} \overline{0} 1^{7} .
\end{aligned}
$$


Then, considering this chain with coefficients in $\mathbb{Z}_{4}\left[t^{ \pm 1}\right] /\left(1-t^{2}\right)^{4}$, we get

$$
\bar{\partial} z_{8}=2\left(1-t^{2}\right)^{2} z_{4} x_{2}+2 t^{4}\left(1+t^{4}\right) x_{3},
$$

and dividing by two we get the following cycle in $H_{7}\left(\mathrm{~B}(16,8,8), \mathbb{F}_{2}\right)$ :

$$
\beta_{2}\left(\tilde{x}_{3}\right)=\tilde{x}_{2}^{2}+t^{4}\left(1-t^{2}\right)^{2} \pi_{*}\left(x_{3}\right) .
$$

Notice that both $\tilde{x}_{2}^{2}$ and $t^{4}\left(1-t^{2}\right)^{2} \pi_{*}\left(x_{3}\right)$ generate a submodule of $H_{7}\left(\mathrm{~B}(16,8,8), \mathbb{F}_{2}\right)$ that is isomorphic to $\mathbb{F}_{2}\left[t^{ \pm 1}\right] /\left(1-t^{2}\right)^{2}$. It follows that the kernel of the map

$$
\beta_{2}: H_{8}\left(\mathrm{~B}(16,8,8), \mathbb{F}_{2}\right) \rightarrow H_{7}\left(\mathrm{~B}(16,8,8), \mathbb{F}_{2}\right)
$$

is generated by the cycle $\left(1-t^{2}\right)^{2} \widetilde{x}_{3}$ and hence $\operatorname{ker} \beta_{2 \mid H_{8}} \simeq \mathbb{F}_{2}\left[t^{ \pm 1}\right] /\left(1-t^{2}\right)^{2}$ is an $\mathbb{F}_{2}$ vector space of dimension 4 . Now recall that, according to Theorem 1.2, $\operatorname{dim} H_{8}(\mathrm{~B}(16,8,8), \mathbb{Q})=2$. The Bockstein spectral sequence then implies that there should be an element in $H_{7}(\mathrm{~B}(16,8,8), \mathbb{Z})$ that has at least 4-torsion.

\subsection{Stabilization}

There is a natural inclusion $j_{r}: \mathrm{B}(2 e, e, r) \hookrightarrow \mathrm{B}(2 e, e, r+1)$. The map $j_{r}$ is induced by the embedding of diagrams. Moreover it is induced by the analogous natural stabilization map for the Artin groups of type $\mathbf{B}_{r}$ as we have the commuting diagram

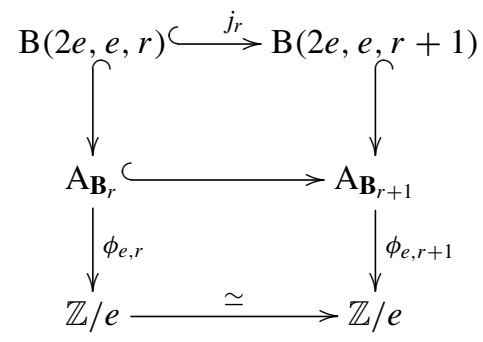

Hence the direct limit $\mathrm{B}(2 e, e, \infty):=\lim _{r} \mathrm{~B}(2 e, e, r)$ is a natural union of groups. The inclusion map $j_{r}$ corresponds to the inclusion map for the algebraic complexes, hence we can compute the homology of the group $\mathrm{B}(2 e, e, \infty)$ using the direct limit of the complexes for $\mathrm{B}(2 e, e, r)$. It is easy to describe, as a corollary of Theorems 4.9 and 4.15 , the homology of the group $\mathrm{B}(2 e, e, \infty)$. It turns out that the stable homology does not depend on the parameter $e$.

Let

$$
s A_{\infty}\left(\mathbb{F}_{2}\right)=\mathbb{F}_{2}\left[\bar{x}_{1}, \bar{x}_{2}, \bar{x}_{3}, \ldots\right]
$$

be the ring of polynomials in the variables $\bar{x}_{i}$, considered as an $\mathbb{F}_{2}\left[t^{ \pm 1}\right]$-module, where $t$ acts trivially, and graded by $\operatorname{dim} \bar{x}_{i}=2^{i}-1$. For an odd prime $p$ we define

$$
s A_{\infty}\left(\mathbb{F}_{p}\right)=\mathbb{F}_{p}\left[\bar{y}_{1}, \bar{y}_{2}, \bar{y}_{3}, \ldots\right] \otimes \Lambda\left[\bar{x}_{0}, \bar{x}_{1}, \bar{x}_{2}, \ldots\right],
$$


the tensor product of the ring of polynomials in the variables $\bar{y}_{i}$ and the exterior algebra in the variables $\bar{x}_{i}$, considered as an $\mathbb{F}_{p}\left[t^{ \pm 1}\right]$-module, where $t$ acts by $(-1)$-multiplication, and graded by $\operatorname{dim} \bar{x}_{i}=2 p^{i}-1, \operatorname{dim} \bar{y}_{i}=2 p^{i}-2$.

Note that the ring $\mathbb{F}_{2}\left[\bar{x}_{1}, \bar{x}_{2}, \bar{x}_{3}, \ldots\right]$ (resp. $\mathbb{F}_{p}\left[\bar{y}_{1}, \bar{y}_{2}, \bar{y}_{3}, \ldots\right] \otimes \Lambda\left[\bar{x}_{0}, \bar{x}_{1}, \bar{x}_{2}, \ldots\right]$ for $p>2$ ) is isomorphic to the stable homology of the braid group, $H_{*}\left(\operatorname{Br}(\infty), \mathbb{F}_{2}\right.$ ) (resp. $\left.H_{*}\left(\operatorname{Br}(\infty), \mathbb{F}_{p}\right)\right)$. The ring structure is induced by the map

$$
\operatorname{Br}\left(n_{1}\right) \times \operatorname{Br}\left(n_{2}\right) \rightarrow \operatorname{Br}\left(n_{1}+n_{2}\right) .
$$

From the presentation of the groups $\mathrm{B}(d e, e, r)$ and $\mathrm{Br}(r)$ one can define in a similar way an injective homomorphism

$$
\mathrm{B}\left(d e, e, r_{1}\right) \times \mathrm{Br}\left(r_{2}+1\right) \rightarrow \mathrm{B}\left(d e, e, r_{1}+r_{2}\right)
$$

induced by the standard inclusion $\mathrm{B}\left(d e, e, r_{1}\right) \hookrightarrow \mathrm{B}\left(d e, e, r_{1}+r_{2}\right)$ and by the map

$$
\mathrm{Br}\left(r_{2}+1\right) \rightarrow \mathrm{B}\left(d e, e, r_{1}+r_{2}\right)
$$

defined on the generators by $\xi_{i} \mapsto \xi_{r_{1}+i}$, according to the presentations given in Section 4.1. The homomorphism (7) induces on $H_{*}\left(\mathrm{~B}(2 e, e, \infty), \mathbb{F}_{p}\right)$ a natural module structure over the ring $H_{*}\left(\operatorname{Br}(\infty), \mathbb{F}_{p}\right)$ for any prime $p$.

The description of the stable homology is the following:

Corollary 4.17. The homology $H_{*}\left(\mathrm{~B}(2 e, e, \infty), \mathbb{F}_{2}\right)$ is isomorphic, as a module over the ring $H_{*}\left(\operatorname{Br}(\infty), \mathbb{F}_{2}\right)$, to the direct limit $\lim _{r} H_{*}\left(\mathrm{~B}(2 e, e, r), \mathbb{F}_{2}\right)$ and is given by the tensor product

$$
s A_{\infty}\left(\mathbb{F}_{2}\right) \otimes \mathbb{F}_{2}\left[w_{1}\right]
$$

where $w_{1}$ is an element of (homological) dimension 1. Moreover the homology groups $H_{*}\left(\mathrm{~B}(2 e, e, r), \mathbb{F}_{2}\right)$ stabilize and the canonical morphism to the direct limit

$$
H_{i}\left(\left(\mathrm{~B}(2 e, e, r), \mathbb{F}_{2}\right) \rightarrow H_{i}\left(\mathrm{~B}(2 e, e, \infty), \mathbb{F}_{2}\right)\right.
$$

is an isomorphism for $r>2 i$.

Corollary 4.18. Let $p$ be an odd prime. The homology $H_{*}\left(\mathrm{~B}(2 e, e, \infty), \mathbb{F}_{p}\right)$ is isomorphic, as a module over the ring $H_{*}\left(\operatorname{Br}(\infty), \mathbb{F}_{p}\right)$, to $\lim _{r} H_{*}\left(\mathrm{~B}(2 e, e, r), \mathbb{F}_{p}\right)$ and is given by the tensor product

$$
s A_{\infty}\left(\mathbb{F}_{p}\right) \otimes \mathbb{F}_{p}\left[w_{1}\right]
$$

where $w_{1}$ is an element of dimension 1 . Moreover the groups $H_{*}\left(\mathrm{~B}(2 e, e, r), \mathbb{F}_{p}\right)$ stabilize and the canonical morphism to the direct limit

$$
H_{i}\left(\mathrm{~B}(2 e, e, r), \mathbb{F}_{p}\right) \rightarrow H_{i}\left(\mathrm{~B}(2 e, e, \infty), \mathbb{F}_{p}\right)
$$

is an isomorphism for $r>(i-1) \frac{p}{p-1}+2$.

Similar considerations leads to an analogous result for stabilization of rational homology: 
Corollary 4.19. The homology $H_{*}(\mathrm{~B}(2 e, e, \infty), \mathbb{Q})$ is isomorphic, as a module over the ring $H_{*}(\operatorname{Br}(\infty), \mathbb{Q})$, to

$$
\Lambda\left[x_{1}\right] \otimes \mathbb{Q}\left[w_{1}\right]
$$

where $x_{1}$ and $w_{1}$ are elements of dimension 1 . Moreover the groups $H_{*}(\mathrm{~B}(2 e, e, r), \mathbb{Q})$ stabilize and the canonical morphism to the direct limit

$$
H_{i}(\mathrm{~B}(2 e, e, r), \mathbb{Q}) \rightarrow H_{i}(\mathrm{~B}(2 e, e, \infty), \mathbb{Q})
$$

is an isomorphism for $r>i+1$.

Remark 4.20. We recall that the homology groups $H_{*}\left(\mathrm{~B}(2 e, e, r), \mathbb{F}_{p}\right)$ given in Corollaries 4.17-4.19 should not be considered as rings, as we describe them only as $H_{*}\left(\operatorname{Br}(\infty), \mathbb{F}_{p}\right)$-modules. Moreover these groups have a natural structure of coalgebra, dual to the ring structure in cohomology given by the cup product, but we have not been able to compute it.

We can then write the Poincaré polynomial for the stable homology. We define

$$
\begin{aligned}
& P_{S A_{\infty}\left(\mathbb{F}_{2}\right)}(u):=\prod_{j \geq 1} \frac{1}{1-u^{2^{j}-1}}, \\
& P_{S A_{\infty}\left(\mathbb{F}_{p}\right)}(u):=\prod_{i \geq 1} \frac{1}{1-u^{2 p^{i}-2}} \prod_{j \geq 0}\left(1+u^{2 p^{j}-1}\right) .
\end{aligned}
$$

Corollary 4.21. For any prime $p$ the Poincaré polynomial for $H_{*}\left(\mathrm{~B}(2 e, e, \infty), \mathbb{F}_{p}\right)$ is

$$
P_{s A_{\infty}\left(\mathbb{F}_{p}\right)}(u) \frac{1}{1-u} .
$$

The Poincaré polynomial for $H_{*}(\mathrm{~B}(2 e, e, \infty), \mathbb{Q})$ is

$$
\frac{1+u}{1-u}
$$

In [Seg73] Segal proved that the spaces $K(\operatorname{Br}(\infty), 1)$ and $\Omega_{0}^{2} S^{2} \simeq \Omega^{2} S^{3}$ have the same homology:

Theorem 4.22 ([Seg73]). There exists a map $K(\operatorname{Br}(\infty), 1) \rightarrow \Omega_{0}^{2} S^{2}$ inducing the following isomorphism in homology:

$$
H_{*}(\operatorname{Br}(\infty), G) \simeq H_{*}\left(\Omega^{2} S^{3}, G\right)
$$

for any group $G$ of coefficients with trivial action of $\operatorname{Br}(\infty)$.

In the same way [Fuk74] Fuks discovered the following analog of Segal's theorem for the Artin groups of type $\mathbf{B}$ and $\mathbf{D}$. We denote by $S^{3}\{2\}$ the homotopy fibre of a map of degree 2 from $S^{3}$ to itself. 
Theorem 4.23 ([Fuk74]). There exist maps

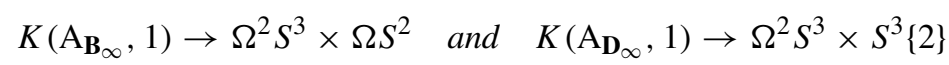

that induce homology isomorphisms.

The homology of the spaces $\Omega^{2} S^{3}, \Omega S^{2}$ and $S^{3}\{2\}$ is well known. In particular, the integral homology of $\Omega^{2} S^{3}, \Omega S^{2}$ has no $p^{2}$-torsion for any prime $p$.

The result of Fuks about $A_{\mathbf{B}_{\infty}}$ agrees with our computation for the homology of $\mathrm{B}(2,1, \infty)$ and we can use it in order to provide more information on the integer homology of $\mathrm{B}(2 e, e, \infty)$.

Proposition 4.24. For any e we have the following isomorphism:

$$
H_{*}(\mathrm{~B}(2,1, \infty), \mathbb{Z}) \simeq H_{*}(\mathrm{~B}(2 e, e, \infty), \mathbb{Z}) .
$$

In particular the integral homology of $\mathrm{B}(2 e, e, \infty)$ has no $p^{2}$-torsion. Moreover the homology groups $H_{*}(\mathrm{~B}(2 e, e, r), \mathbb{Z})$ stabilize and the canonical morphism to the direct limit

$$
H_{i}(\mathrm{~B}(2 e, e, r), \mathbb{Z}) \rightarrow H_{i}(\mathrm{~B}(2 e, e, \infty), \mathbb{Z})
$$

is an isomorphism for $r>2 i+2$.

Proof. Let us fix $i>0$ and $e>1$. We shall prove the isomorphism

$$
H_{i}(\mathrm{~B}(2,1, \infty), \mathbb{Z}) \simeq H_{i}(\mathrm{~B}(2 e, e, \infty), \mathbb{Z})
$$

To do this we shall provide an isomorphism between the free (abelian) quotients by the torsion subgroups and another one between the $p$-parts for any prime $p$. (We define the p-part of an abelian group $M$ to be the subgroup $\left\{m \in M \mid \exists k \in \mathbb{N}, p^{k} m=0\right\}$.) As $H_{i}(\mathrm{~B}(2,1, \infty), \mathbb{Z})$ is finitely generated, this will prove that $H_{i}(\mathrm{~B}(2 e, e, \infty), \mathbb{Z})$ is finitely generated too, hence that the two groups are isomorphic. In the process we shall prove that the stabilization maps for the $H_{i}(\mathrm{~B}(2 e, e, r), \mathbb{Z})$ are isomorphisms on the torsion subgroups and on the free quotient for $r>2 i+2$.

Recall from Proposition 2.2 that we have the isomorphisms

$$
\mathrm{B}(2 e, e, r) \simeq \mathrm{B}(2(r \pm e), r \pm e, r) .
$$

We assume that we choose $r$ large enough that, for any $a$ and for any prime $p$, $H_{i}\left(\mathrm{~B}(2 a, a, r), \mathbb{F}_{p}\right), H_{i}(\mathrm{~B}(2 a, a, r), \mathbb{Q})$ and $H_{i}(\mathrm{~B}(2,1, r), \mathbb{Z})$ stabilize. This means that the maps $H_{i}\left(\mathrm{~B}(2 a, a, r), \mathbb{F}_{p}\right) \rightarrow H_{i}\left(\mathrm{~B}\left(2 a, a, r^{\prime}\right), \mathbb{F}_{p}\right), H_{i}(\mathrm{~B}(2 a, a, r), \mathbb{Q}) \rightarrow$ $H_{i}\left(\mathrm{~B}\left(2 a, a, r^{\prime}\right), \mathbb{Q}\right)$ and $H_{i}(\mathrm{~B}(2,1, r), \mathbb{Z}) \rightarrow H_{i}\left(\mathrm{~B}\left(2,1, r^{\prime}\right), \mathbb{Z}\right)$, with $r^{\prime}>r$, are isomorphisms. For this it is enough to take $r>2 i+2$.

We start by considering the maps

$$
\left(j_{r}\right)_{*}: H_{i}(\mathrm{~B}(2 e, e, r), \mathbb{Z}) \rightarrow H_{i}(\mathrm{~B}(2 e, e, r+1), \mathbb{Z}) .
$$

We fix $x, y$ generating a maximal free subgroup $\mathbb{Z}^{2} \subset H_{i}(\mathrm{~B}(2 e, e, r), \mathbb{Z})$. Moreover we choose a collection of maximal free subgroups $\mathbb{Z}^{2} \subset H_{i}(\mathrm{~B}(2 e, e, r+1), \mathbb{Z})$ with generators $w, z$ such that the image of $x, y$ lies inside the group generated by $w, z$. 
The restriction of $\left(j_{r}\right)_{*}$ to $\langle x, y\rangle=\mathbb{Z}^{2}$ defines a map

$$
\left(j_{r}\right)_{*}^{(0)}: \mathbb{Z}^{2} \rightarrow \mathbb{Z}^{2}
$$

whose image lies in the group $\langle w, z\rangle=\mathbb{Z}^{2}$. This map is injective by stabilization of the rational homology. We claim that this map is also surjective. Otherwise, let $d$ be the determinant of $\left(j_{r}\right)_{*}^{(0)}$ and let $p$ be a prime that divides $d$. Let $\bar{x}, \bar{y}$ be the images of $x, y$ through the natural homomorphism $H_{i}(\mathrm{~B}(2 e, e, r), \mathbb{Z}) \rightarrow H_{i}\left(\mathrm{~B}(2 e, e, r), \mathbb{F}_{p}\right)$. The commuting diagram

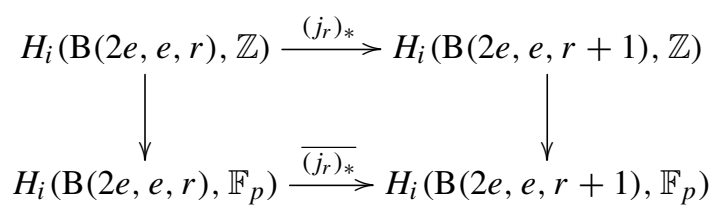

implies that the map $\overline{\left(j_{r}\right)_{*}}: H_{i}\left(\mathrm{~B}(2 e, e, r), \mathbb{F}_{p}\right) \rightarrow H_{i}\left(\mathrm{~B}(2 e, e, r+1), \mathbb{F}_{p}\right)$ is not injective, and this is a contradiction. As a consequence we can choose a maximal free subgroup $F_{s} \subset H_{i}(\mathrm{~B}(2 e, e, s), \mathbb{Z})$ for each $s \geq r$ with $j_{s}\left(F_{S}\right) \subset F_{s+1}$, which induces isomorphisms

$$
H_{i}(\mathrm{~B}(2 e, e, s), \mathbb{Z}) / \text { torsion } \stackrel{\simeq}{\rightarrow} H_{i}(\mathrm{~B}(2 e, e, s+1), \mathbb{Z}) / \text { torsion } \simeq \mathbb{Z}^{2},
$$

hence $H_{i}(\mathrm{~B}(2 e, e, \infty), \mathbb{Z}) /$ torsion $\simeq \mathbb{Z}^{2}$.

Now we can consider the following composition of an inclusion and isomorphisms:

$\mathrm{B}(2 e, e, r) \stackrel{\simeq}{\rightarrow} \mathrm{B}(2(e+r), e+r, r) \hookrightarrow \mathrm{B}(2(e+r), e+r, e+r-1) \stackrel{\simeq}{\rightarrow} \mathrm{B}(2,1, e+r-1)$.

In a similar way consider the composition

$$
\begin{aligned}
& \mathrm{B}(2,1, r) \hookrightarrow \mathrm{B}\left(2,1, r^{\prime}-e+1\right) \\
& \quad \stackrel{\simeq}{\rightarrow} \mathrm{B}\left(2\left(r^{\prime}-e\right), r^{\prime}-e, r^{\prime}-e+1\right) \hookrightarrow \mathrm{B}\left(2\left(r^{\prime}-e\right), r^{\prime}-e, r^{\prime}\right) \stackrel{\simeq}{\rightarrow} \mathrm{B}\left(2 e, e, r^{\prime}\right)
\end{aligned}
$$

where $r^{\prime}$ is large enough that $r^{\prime}-e+1 \geq r$.

In particular we can assume that we have maps $\mathrm{B}(2,1, r) \rightarrow \mathrm{B}\left(2 e, e, r^{\prime}\right)$ and $\mathrm{B}\left(2 e, e, r^{\prime}\right) \rightarrow \mathrm{B}\left(2,1, r^{\prime \prime}\right)$ that induce isomorphisms for $H_{i}$ with coefficients in $\mathbb{F}_{p}$. It follows that also the composition

$$
\mathrm{B}(2,1, r) \rightarrow \mathrm{B}\left(2 e, e, r^{\prime}\right) \rightarrow \mathrm{B}\left(2,1, r^{\prime \prime}\right)
$$

induces an isomorphism $H_{i}\left(\mathrm{~B}(2,1, r), \mathbb{F}_{p}\right) \simeq H_{i}\left(\mathrm{~B}\left(2,1, r^{\prime \prime}\right), \mathbb{F}_{p}\right)$ and hence an isomorphism between the $p$-parts of $H_{i}(\mathrm{~B}(2,1, r), \mathbb{Z})$ and $H_{i}\left(\mathrm{~B}\left(2,1, r^{\prime \prime}\right), \mathbb{Z}\right)$. From the previous description and because we know that there is no $p^{2}$-torsion in the integral homology of $\mathrm{B}(2,1, \infty)$ we can assume that $H_{i}(\mathrm{~B}(2,1, r), \mathbb{Z}) \simeq H_{i}\left(\mathrm{~B}\left(2,1, r^{\prime \prime}\right), \mathbb{Z}\right) \simeq \mathbb{Z}_{p}^{k} \oplus \mathbb{Z}^{2} \oplus T_{r}$ where $T_{r}$ is a finite group without $p$-torsion. Moreover we have

$$
H_{i}\left(\mathrm{~B}\left(2 e, e, r^{\prime}\right), \mathbb{Z}\right) \simeq \bigoplus_{h=1}^{k} \mathbb{Z}_{p^{l_{h}}} \oplus \mathbb{Z}^{2} \oplus T_{r}^{\prime}
$$


for some collection $l_{h}$ of positive integers and $T_{r}^{\prime}$ a finite group without $p$-torsion. The maps $H_{i}(\mathrm{~B}(2,1, r), \mathbb{Z}) \stackrel{f_{1}}{\rightarrow} H_{i}\left(\mathrm{~B}\left(2 e, e, r^{\prime}\right), \mathbb{Z}\right)$ and $H_{i}\left(\mathrm{~B}\left(2 e, e, r^{\prime}\right), \mathbb{Z}\right) \stackrel{f_{2}}{\rightarrow}$ $H_{i}\left(\mathrm{~B}\left(2,1, r^{\prime \prime}\right), \mathbb{Z}\right)$ induce homomorphisms between the $p$-parts

$$
f_{1}^{(p)}: \mathbb{Z}_{p}^{k} \rightarrow \bigoplus_{h=1}^{k} \mathbb{Z}_{p^{l_{h}}} \quad \text { and } \quad f_{2}^{(p)}: \bigoplus_{h=1}^{k} \mathbb{Z}_{p^{l_{h}}} \rightarrow \mathbb{Z}_{p}^{k}
$$

As $f_{2}^{(p)} \circ f_{1}^{(p)}$ is an isomorphism, it follows that for all $h=1, \ldots, k$ we have $l_{h}=1$ and hence $\mathbb{Z}_{p}^{k} \simeq \bigoplus_{h=1}^{k} \mathbb{Z}_{p^{l_{h}}}$.

As the map $\overline{\left(j_{r}\right)_{*}}: H_{i}\left(\mathrm{~B}(2 e, e, r), \mathbb{F}_{p}\right) \rightarrow H_{i}\left(\mathrm{~B}(2 e, e, r+1), \mathbb{F}_{p}\right)$ is an isomorphism induced by $\left(j_{r}\right)_{*}: H_{i}(\mathrm{~B}(2 e, e, r), \mathbb{Z}) \rightarrow H_{i}(\mathrm{~B}(2 e, e, r+1), \mathbb{Z})$, it follows that $\left(j_{r}\right)_{*}$ is injective on the $p$-part of $H_{i}(\mathrm{~B}(2 e, e, r), \mathbb{Z})$. Since the $p$-parts of both sides are finite and have the same cardinality, $\left(j_{r}\right)_{*}$ induces an isomorphism on the $p$-parts. It follows that the $p$-part of $H_{i}(\mathrm{~B}(2 e, e, \infty), \mathbb{Z})$ is isomorphic to $\mathbb{Z}_{p}^{k}$, that is, to the $p$-part of $H_{i}(\mathrm{~B}(2,1, \infty), \mathbb{Z})$. The result follows.

Remark 4.25. We point out that in general the isomorphism for stable homology given in Proposition 4.24 is not induced by the natural inclusion

$$
\mathrm{B}(2 e, e, r) \hookrightarrow \mathrm{B}(2,1, r) .
$$

We can prove this by considering homology with coefficients in a finite field $\mathbb{F}_{p}$. First we recall that from the Shapiro Lemma the morphism

$$
\left(\pi_{e}\right)_{*}: H_{*}\left(\mathrm{~B}(2 e, e, r), \mathbb{F}_{p}\right) \rightarrow H_{*}\left(\mathrm{~B}(2,1, r), \mathbb{F}_{p}\right)
$$

induced by the inclusion (8) corresponds to the morphism

$$
H_{*}\left(\mathrm{~A}_{\mathbf{B}_{r}}, \mathbb{F}_{p}[t] /\left(1-(-t)^{e}\right)\right) \rightarrow H_{*}\left(\mathrm{~A}_{\mathbf{B}_{r}}, \mathbb{F}_{p}[t] /(1+t)\right)
$$

induced by the natural projection

$$
\pi_{e}: \mathbb{F}_{p}[t] /\left(1-(-t)^{e}\right) \rightarrow \mathbb{F}_{p}[t] /(1+t) .
$$

Now consider the following commuting diagram, where the horizontal lines are exact:

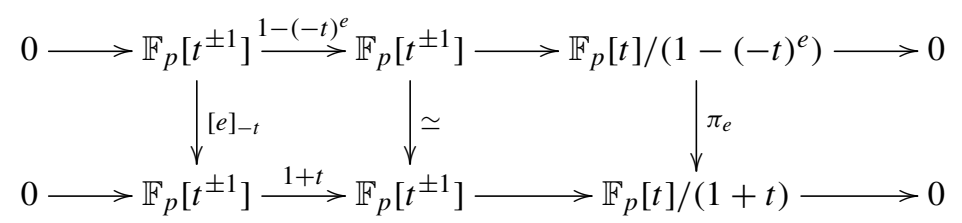

where we recall the notation $[e]_{-t}=1+(-t)+\cdots+(-t)^{e-1}$. Now assume that $r$ and $i$ are such that the homology groups $H_{i}\left(\mathrm{~A}_{\mathbf{B}_{r}}, \mathbb{F}_{p}\left[t^{ \pm 1}\right]\right), H_{i-1}\left(\mathrm{~A}_{\mathbf{B}_{r}}, \mathbb{F}_{p}\left[t^{ \pm 1}\right]\right)$, $H_{i}\left(\mathrm{~B}(2 e, e, r), \mathbb{F}_{p}\right)$ and $H_{i}\left(\mathrm{~B}(2,1, r), \mathbb{F}_{p}\right)$ are stable. In particular $-t$ acts trivially, hence 
multiplication by $1+t$ and by $1-(-t)^{e}$ are zero maps, while multiplication by $[e]_{-t}$ corresponds to multiplication by $e$.

The following induced diagram for homology

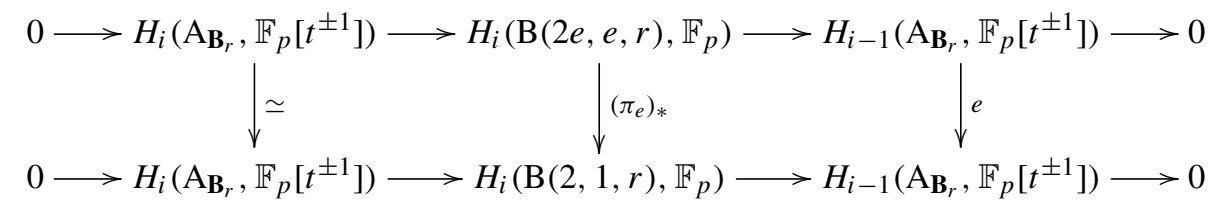

shows that when $p \mid e$ and the group $H_{i-1}\left(\mathrm{~A}_{\mathbf{B}_{r}}, \mathbb{F}_{p}\left[t^{ \pm 1}\right]\right)$ is non-trivial, the map $\left(\pi_{e}\right)_{*}$ has non-trivial kernel.

\section{Complexes from Garside theory}

We recall a few homological constructions from the theory of Garside monoids and groups. Recall that a Garside group $G$ is the group of fractions of a Garside monoid $M$, where Garside means that $M$ satisfies several conditions for which we refer to [DP99]. In particular, $M$ admits (left) lcm's, and contains a special element, called the Garside element. We denote by $\mathcal{X}$ the set of atoms in $M$, assumed to be finite. The homology of $G$ coincides with the homology of $M$. Garside theory provides two useful resolutions of $\mathbb{Z}$ by free $\mathbb{Z} M$-modules.

The first one was defined in [CMW04]. Another one, with more complicated differential but a smaller number of cells, has been defined in [DL03].

\subsection{The Dehornoy-Lafont complex}

Let $M$ be a Garside monoid with a finite set $\mathcal{X}$ of atoms. We choose an arbitrary linear order $<$ on $\mathcal{X}$. For $m \in M$, denote by $\operatorname{md}(m)$ the smaller element in $\mathcal{X}$ which divides $m$ on the right $(m=a \operatorname{md}(m)$ for some $a \in M)$. Recall that $\operatorname{lcm}(x, y)$ for $x, y \in M$ denotes the least common multiple on the left, that is, $v=g x=h y$ implies $v=j \operatorname{lcm}(x, y)$ for some $j \in M$. If $A=(x, B)$ is a list of elements in $M$ we define inductively $\operatorname{lcm}(A)=$ $\operatorname{lcm}(x, \operatorname{lcm}(B))$.

An $n$-cell is an $n$-tuple $\left[x_{1}, \ldots, x_{n}\right]$ of elements in $\mathcal{X}$ such that $x_{1}<\cdots<x_{n}$ and $x_{i}=\operatorname{md}\left(\operatorname{lcm}\left(x_{i}, x_{i+1}, \ldots, x_{n}\right)\right)$. Let $\mathcal{X}_{n}$ denote the set of all such $n$-cells. By convention $\mathcal{X}_{0}=\{[\emptyset]\}$. The set $C_{n}$ of $n$-chains is the free $\mathbb{Z} M$-module with basis $\mathcal{X}_{n}$. The differential $\partial_{n}: C_{n} \rightarrow C_{n-1}$ is defined recursively through two auxiliary $\mathbb{Z}$-module homomorphisms $s_{n}: C_{n} \rightarrow C_{n+1}$ and $r_{n}: C_{n} \rightarrow C_{n}$. Let $[\alpha, A]$ be an $(n+1)$-cell, with $\alpha \in \mathcal{X}$ and $A$ an $n$-cell. We let $\alpha_{/ A}$ denote the unique element in $M$ such that $\left(\alpha_{/ A}\right) \operatorname{lcm}(A)=\operatorname{lcm}(\alpha, A)$. The defining equations for $\partial$ and $r$ are

$$
\partial_{n+1}[\alpha, A]=\alpha_{/ A}[A]-r_{n}(\alpha / A[A]), \quad r_{n+1}=s_{n} \circ \partial_{n+1}, \quad r_{0}(m[\emptyset])=[\emptyset] .
$$


In order to define $s_{n}$, we say that $x[A]$ for $x \in M$ and $A$ an $n$-cell is irreducible if $x=1$ and $A=\emptyset$, or if $\alpha=\operatorname{md}(x \operatorname{lcm}(A))$ coincides with the first coefficient in $A$. In that case, we let $s_{n}(x[A])=0$, and otherwise

$$
s_{n}(x[A])=y[\alpha, A]+s_{n}\left(y r_{n}\left(\alpha_{/ A}[A]\right)\right) \quad \text { with } x=y \alpha_{/ A} .
$$

\subsection{The Charney-Meyer-Wittlesey complex}

Let again $G$ denote the group of fractions of a Garside monoid $M$, with Garside element $\Delta$. Let $\mathcal{D}$ denote the set of simple elements in $M$, that is, the (finite) set of proper divisors of $\Delta$. We let $\mathcal{D}_{n}$ denote the set of $n$-tuples $\left[\mu_{1}|\ldots| \mu_{n}\right]$ such that each $\mu_{i}$ as well as the product $\mu_{1} \cdots \mu_{n}$ lie in $\mathcal{D}$. The differential from the free $\mathbb{Z} M$-modules $\mathbb{Z} M \mathcal{D}_{n}$ to $\mathbb{Z} M \mathcal{D}_{n-1}$ is given by

$$
\begin{aligned}
& \partial_{n}\left[\mu_{1}|\ldots| \mu_{n}\right] \\
& \quad=\mu_{1}\left[\mu_{2}|\ldots| \mu_{n}\right]+\sum_{i=1}^{n-1}(-1)^{i}\left[\mu_{1}, \ldots, \mu_{i} \mu_{i+1}, \ldots, \mu_{n}\right]+(-1)^{n}\left[\mu_{1}|\ldots| \mu_{n-1}\right] .
\end{aligned}
$$

This complex in general has larger cells than the previous one. Its main advantage for us is that the definition of the differential is simpler, and does not involve many recursion levels. Both complexes will be used in Section 5.3 below.

\subsection{Application to the exceptional groups}

When $W$ is well-generated, meaning that it can be generated by $n$ reflections, where $n$ denotes the rank of $W$, then $B$ is the group of fractions of (usually) several Garside monoids that generalize the Birman-Ko-Lee monoid of the usual braid groups. These monoids have been introduced by D. Bessis [Bes07] and called there dual braid monoids. They are determined by the choice of a so-called Coxeter element $c$. Such an element is regular, meaning that it admits only one eigenvalue different from 1 with the corresponding eigenvector outside the reflection hyperplanes. A Coxeter element is a regular element with eigenvalue $\exp (2 \mathrm{i} \pi / h)$, where $h$ denotes the (generalized) Coxeter number for $W$, namely its highest degree as a reflection group.

The corresponding Garside monoid $M_{c}$ is then generated by some set $R_{c}$ of braided reflections with relations of the form $r r^{\prime}=r^{\prime} r^{\prime \prime}$ (see [Bes07] for more details). The above Charney-Meyer-Wittlesey complexes for these monoids have been implemented by Jean Michel and the second author, using the (development version of) the CHEVIE package for GAP3 (see [Mic]). The chosen Coxeter elements are indicated in Table 7, in terms of the usual presentations of these groups (see [BMR98] for an explanation of the diagrams).

Using the HAP package for GAP4 we then obtained the homologies described in Table 11 (we recall in Table 12 the ones obtained earlier by Salvetti for the Coxeter groups) except for the groups $G_{12}, G_{13}, G_{22}, G_{31}$, which are not well-generated, as well as $H_{3}(B, \mathbb{Z})$ of type $G_{33}$. When $W$ has type $G_{13}$, the group $B$ is the same as when $W$ has 
Table 7. Coxeter elements for dual monoids.

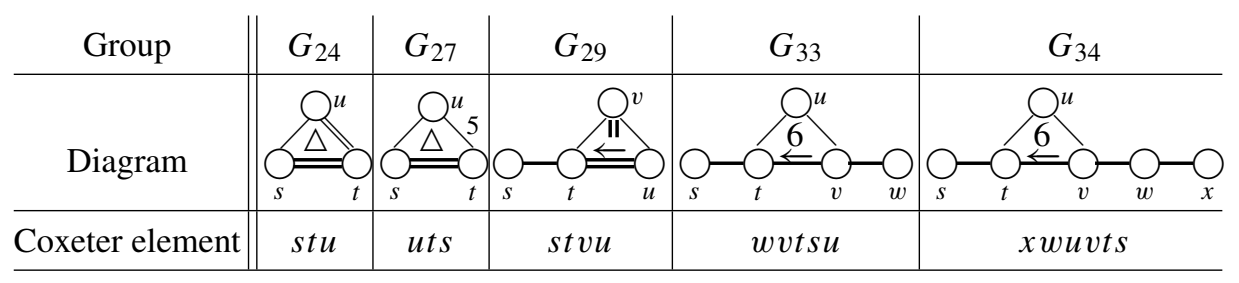

Coxeter type $I_{2}(6)$, and the result is known. For $G_{12}$ and $G_{22}$ one can use the Garside monoids introduced by M. Picantin [Pic00].

A complex for $G_{31}$ can be obtained from the theory of Garside categories by considering it as the centralizer of some regular element in the Coxeter group $E_{8}$. This viewpoint was used in [Bes07] to prove that the corresponding spaces $X$ and $X / W$ are $K(\pi, 1)$. More precisely, a simplicial complex (reminiscent of the Charney-Meyer-Wittlesey complex described above) is constructed in [Bes07], which is homotopically equivalent to $X / W$. From this construction, we got a complex from an implementation by Jean Michel in CHEVIE.

However, for $G_{31}, G_{33}$ and $G_{34}$, the complexes obtained are too large to be dealt with completely through usual computers and software. Those missing for $G_{31}$ and $G_{33}$ are the middle homology $H_{2}(B, \mathbb{Z})$ for $G_{31}$ and $H_{3}(B, \mathbb{Z})$ for $G_{33}$. The Dehornoy-Lafont complex for $G_{33}$ is however computable in reasonable time, and its small size enables one to compute the whole homology by standard methods. For $G_{31}$, for which there is so far no construction analogous to the Dehornoy-Lafont complex, we used the following method for computing $\mathrm{H}_{2}(B, \mathbb{Z})$.

We first get $H_{2}(B, \mathbb{Q})=0$ by computing the second Betti number from the lattice. Indeed, recall from [OT92, Cor. 6.17, p. 223] that the Betti numbers of $X / W$ can be in principle computed from the lattice of the arrangement. More precisely, the second Betti number of $X / W$ is given by $\sum_{Z \in T_{2}}\left|\mathcal{H}_{Z} / W_{Z}\right|-1$ where $T_{i}$ is a system of representatives modulo $W$ of codimension $i$ subspaces in the arrangement lattice; for $Z$ such a subspace, $\mathcal{H}_{Z}=\{H \in \mathcal{A} \mid H \supset Z\}, W_{Z}=\{w \in W \mid w(Z)=Z\}$. More generally, the $i$-th Betti number is given by

$$
(-1)^{i} \sum_{Z \in T_{i}} \sum_{\sigma \in U_{Z}}(-1)^{d(\sigma)}
$$

where $U_{Z}$ is the set of classes modulo $W$ of the set of simplices of the augmented Folkman complex of the lattice $\mathcal{A}_{z}$, and $d(\sigma)$ denotes the dimension of a cell. The Folkman complex of a lattice is defined (see [OT92]) as the complex of the poset obtained by removing the minimal and maximal elements of the lattice; when the maximal codimension of the lattice is 1, then the Folkman complex is empty. The augmented Folkman complex is defined by adding to the Folkman complex one $G$-invariant simplex of dimension -1 . In the case of $G_{31}$ the computation of this formula is doable and we get 0 for the second Betti number.

We then reduce our original complex mod $p^{r}$, for $p^{r}$ small enough so that we can encode each matrix entry inside one byte. Then we wrote a $\mathrm{C}$ program to compute 
$H_{2}\left(B, \mathbb{Z}_{4}\right)=H_{2}\left(B, \mathbb{Z}_{2}\right)=\mathbb{Z}_{2}, H_{2}\left(B, \mathbb{Z}_{9}\right)=H_{2}\left(B, \mathbb{Z}_{3}\right)=\mathbb{Z}_{3}$ and $H_{2}\left(B, \mathbb{Z}_{5}\right)=0$ (the matrix of $d_{3}$ has size $11065 \times 15300$ ). Since $G_{31}$ has order $2^{10} \cdot 3^{2} \cdot 5$ and $H_{*}(P)$ is torsion-free, for $p \notin\{2,3,5\}$ we have $H_{2}\left(B, \mathbb{Z}_{p}\right)=H_{2}\left(P, \mathbb{Z}_{p}\right)^{W}=\left(H_{2}(P, \mathbb{Z})^{W}\right) \otimes \mathbb{Z}_{p}$. But $0=H_{2}(B, \mathbb{Q})=H_{2}(P, \mathbb{Q})^{W}=H_{2}(P, \mathbb{Z})^{W} \otimes \mathbb{Q}$, whence $H_{2}(P, \mathbb{Z})^{W}=0$ and $H_{2}\left(B, \mathbb{Z}_{p}\right)=0$. Now $H_{1}(B, \mathbb{Z})=\mathbb{Z}$ is torsion-free, hence $H_{2}\left(B, \mathbb{Z}_{n}\right) \simeq H_{2}(B, \mathbb{Z}) \otimes \mathbb{Z}_{n}$ for any $n$ by the Universal Coefficients Theorem. Since $H_{2}(B, \mathbb{Z})$ is a $\mathbb{Z}$-module of finite type this yields $H_{2}(B, \mathbb{Z})=\mathbb{Z}_{6}$ and completes the computation for $G_{31}$.

\subsection{Embeddings between Artin-like monoids}

We end this section by proving a few lemmas concerning submonoids, which will be helpful in computing differentials in concrete cases.

We consider Garside monoids with set of generators $S$ and endowed with a length function, that is, a monoid morphism $\ell: M \rightarrow \mathbb{N}=\mathbb{Z}_{\geq 0}$ such that $\ell(x)=0 \Leftrightarrow x=1$ and $\ell(s)=1$ for all $s \in S$. We consider the divisibility relation on the left (that is, $U \mid V$ means $\exists m V=U m$ ) and recall that such a monoid admits lcm's (on the left).

Let $M, N$ be two such monoids, and $\varphi: M \rightarrow N$ a monoid morphism such that

(1) $\forall s \in S \varphi(s) \neq 1$,

(2) $\forall s, t \in S \operatorname{lcm}(\varphi(s), \varphi(t))=\varphi(\operatorname{lcm}(s, t))$.

The following results on such morphisms are basically due to J. Crisp [Cri99], who proved them in the case of Artin groups of finite Coxeter type.

Lemma 5.1. Let $U, V \in M$. If $\varphi(U) \mid \varphi(V)$ then $U \mid V$.

Proof. By induction on $\ell(V)$. Since $\ell(\varphi(s)) \geq 1=\ell(s)$ for all $s \in S$, we have $\ell(\varphi(U)) \geq$ $\ell(U)$. Since $\varphi(U) \mid \varphi(V)$, we have $\ell(\varphi(U)) \leq \ell(\varphi(V))$, hence $\ell(U) \leq \ell(\varphi(V))$. Hence $\varphi(V)=1$ implies $\ell(U)=0$ and $U=1$, which settles the case $\ell(V)=0$.

We now assume $\ell(V) \geq 1$. The case $U=1$ being clear, we can assume $U \neq 1$. Then there exist $s, t \in S$ with $s \mid U$ and $t \mid V$. It follows that $\varphi(t) \mid \varphi(V)$ and $\varphi(s)|\varphi(U)| \varphi(V)$, hence $\operatorname{lcm}(\varphi(s), \varphi(t)) \mid \varphi(V)$.

Now $\operatorname{lcm}(s, t)=t m$ for some $m \in M$ and $V=t V^{\prime}$ for some $V^{\prime} \in M$, hence $\varphi(t) \varphi(m) \mid \varphi(V)=\varphi(t) \varphi\left(V^{\prime}\right)$ and this implies $\varphi(m) \mid \varphi\left(V^{\prime}\right)$ by cancellability in $M$. Since $\ell\left(V^{\prime}\right)<\ell(V)$, from the induction assumption follows that $m \mid V^{\prime}$ hence $t m \mid V$ that is $\operatorname{lcm}(s, t) \mid V$. In particular we get $s \mid V$. Writing $V=s V^{\prime \prime}$ and $U=s U^{\prime}$ for some $V^{\prime \prime}, U^{\prime} \in M$, the assumption $\varphi(U) \mid \varphi(V)$ implies $\varphi\left(U^{\prime}\right) \mid \varphi\left(V^{\prime \prime}\right)$ by cancellability, and then $U^{\prime} \mid V^{\prime \prime}$ by the induction assumption. It follows that $U \mid V$, which proves the claim.

The lemma has the following consequence.

Lemma 5.2. The morphism $\varphi: M \rightarrow N$ is injective. If $G_{M}, G_{N}$ denote the groups of fractions of $M, N$, then $\varphi$ can be extended to $\widetilde{\varphi}: G_{M} \hookrightarrow G_{N}$.

Proof. Let $U, V \in M$ with $\varphi(U)=\varphi(V)$. By the lemma we get $U \mid V$ and $V \mid U$. This implies $\ell(U)=\ell(V)$, hence $U=V$. Composing $\varphi: M \rightarrow N$ with the natural morphism $N \hookrightarrow G_{N}$ yields a monoid morphism $M \rightarrow G_{N}$. Since $G_{N}$ is a group, this morphism 
factors through the morphism $M \rightarrow G_{M}$, and this provides $\widetilde{\varphi}: G_{M} \rightarrow G_{N}$. Let $g \in$ Ker $\tilde{\varphi}$. Since $g \in G_{M}$ there exist $a, b \in M$ with $g=a b^{-1}$, hence $\varphi(a)=\varphi(b), a=b$ and $g=1$.

We can now identify $M, N, G_{M}$ to subsets of $G_{N}$. We consider the following extra assumption. We assume that, for all $m \in M, n \in N$, if $n$ divides $m$ in $N$ then $n \in M$.

Lemma 5.3. Under this assumption, $U, V$ in $M$ have the same $l \mathrm{~cm}$ in $M$ and in $N$. Moreover, $M=N \cap G_{M}$.

Proof. Since $\operatorname{lcm}_{M}(U, V)$ divides $U, V$ in $N$, it follows that $\operatorname{lcm}_{N}(U, V)$ divides $\operatorname{lcm}_{M}(U, V)$ in $N$. Conversely, since $\operatorname{lcm}_{N}(U, V)$ divides $U$ in $N$ and $U \in M$, by the assumption we get $\operatorname{lcm}_{N}(U, V) \in M$. From Lemma 5.1 we thus infer that $\operatorname{lcm}_{N}(U, V)$ divides $U$ and $V$ in $M$, hence $\operatorname{lcm}_{M}(U, V)$ divides $\operatorname{lcm}_{N}(U, V)$ in $N$. It follows that $\operatorname{lcm}_{M}(U, V)=\operatorname{lcm}_{N}(U, V)$.

We have $M \subset N \cap G_{M}$. Let $n \in N \cap G_{M}$. Since $n \in G_{M}$ there exist $a, b \in M$ with $n=a b^{-1}$, hence $n b=a \in M$. Hence $n \in N$ divides $a \in M$ in $M$. By the assumption we get $n \in M$ and the conclusion.

\section{The groups $\mathrm{B}(e, e, r)$}

\subsection{The Corran-Picantin monoid}

We denote by $\mathrm{B}(e, e, r)$ for $e \geq 1$ and $r \geq 2$ the braid group associated to the complex reflection group $G(e, e, r)$. Then $\mathrm{B}(e, e, r)$ is the group of fractions of a Garside monoid introduced by R. Corran and M. Picantin (see [CP]). This monoid, which we denote $M(e, e, r)$, has generators (atoms) $t_{0}, t_{1}, \ldots, t_{e-1}, s_{3}, s_{4}, \ldots, s_{r}$ and relations

(1) $t_{i+1} t_{i}=t_{j+1} t_{j}$, with the convention $t_{e}=t_{0}$,

(2) $s_{3} t_{i} s_{3}=t_{i} s_{3} t_{i}$,

(3) $s_{k} t_{i}=t_{i} s_{k}$ for $k \geq 4$,

(4) $s_{k} s_{k+1} s_{k}=s_{k+1} s_{k} s_{k+1}$ for $k \geq 3$,

(5) $s_{k} s_{l}=s_{l} s_{k}$ when $|l-k| \geq 2$.

\subsection{Link with the topological definition}

The connection between this monoid and the group $\mathrm{B}(e, e, r)$ defined as a fundamental group is quite indirect. In [BMR98] a first presentation is obtained by combining embeddings into usual braid groups, fibrations and coverings. The presentation used here is deduced from that one in a purely algebraic manner, by adding generators in order to get a Garside presentation. Although it is folklore, the description of all generators as braided reflections does not appear in the literature (see however [BC06] for a statement without proof in a related context).

In order to provide this connection, we need to recall the way these generators are constructed. For clarity, we stick to the notation of [BMR98]; in that paper, the authors 
introduce four different spaces,

$$
\begin{aligned}
\mathcal{M}(r+1) & =\left\{\left(z_{0}, \ldots, z_{r}\right) \in \mathbb{C}^{r+1} \mid z_{i} \neq z_{j}\right\}, \\
\mathcal{M}^{\#}(m, r) & =\left\{\left(z_{1}, \ldots, z_{r}\right) \in \mathbb{C}^{r}\left|z_{i} \neq 0, z_{i}\right| z_{j} \notin \mu_{m}\right\}, \\
\mathcal{M}(e, r) & =\left\{\left(z_{1}, \ldots, z_{r}\right) \in \mathbb{C}^{r} \mid z_{i} \notin \mu_{e} z_{j}\right\}, \\
\mathcal{M}^{\#}(r) & =\left\{\left(z_{1}, \ldots, z_{r}\right) \in \mathbb{C}^{r} \mid z_{i} \neq 0\right\},
\end{aligned}
$$

where $\mu_{n}$ denotes the set of $n$-th roots of 1 in $\mathbb{C}$. We have a Galois covering $r$ : $\mathcal{M}^{\#}(m, r) \rightarrow \mathcal{M}^{\#}(r)=\mathcal{M}^{\#}(m, r) /\left(\mu_{m}\right)^{r}$, a locally trivial fibration $p: \mathcal{M}(r+1) \rightarrow$ $\mathcal{M}^{\#}(r)$ with fibre $\mathbb{C}$ given by $\left(z_{0}, \ldots, z_{r}\right) \mapsto\left(z_{0}-z_{1}, \ldots, z_{0}-z_{r}\right)$, and a natural action of $\mathfrak{S}_{r}$ on $\mathcal{M}(r+1)$ that leaves the $(r+1)$-st coordinate fixed. We choose a fixed point $x \in \mathcal{M}(r+1) / \mathfrak{S}_{r}$, and a lift $\widetilde{p(x)}$ of $p(x) \in \mathcal{M}^{\#}(r) / \mathfrak{S}_{r}$ in $\mathcal{M}^{\#}(d, r) / G(d, 1, r)=$ $\left(\mathcal{M}(d, r) /\left(\mu_{m}\right)^{r}\right) / \mathfrak{S}_{r}$. We get an isomorphism $\psi: \pi_{1}\left(\mathcal{M}^{\#}(d, r) / G(d, 1, r), \widetilde{p(x)}\right) \rightarrow$ $\pi_{1}\left(\mathcal{M}(r+1) / \mathfrak{S}_{r}, x\right)$ by composing the isomorphisms induced by $r$ and $p$ :

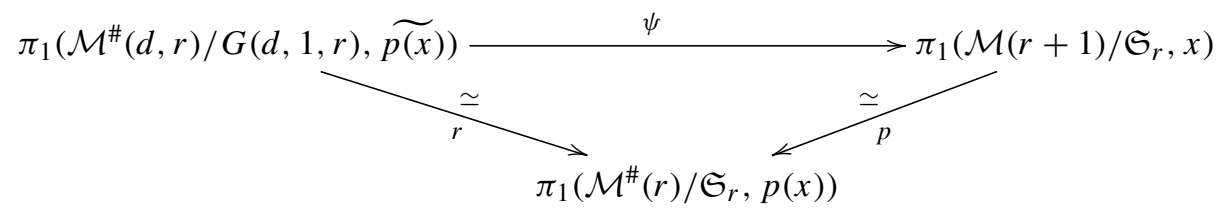

Since $\pi_{1}\left(\mathcal{M}^{\#}(d, r) / G(d, 1, r)\right)=\mathrm{B}(d, 1, r), \psi$ identifies the latter group with $\pi_{1}\left(\mathcal{M}(r+1) / \mathfrak{S}_{r}\right)$. The generators of $\mathrm{B}(d, 1, r)$ are then obtained in [BMR98] by taking the preimages under $\psi$ and the covering of $\mathcal{M}(r+1) / \mathfrak{S}_{r} \rightarrow \mathcal{M}(r+1) / \mathfrak{S}_{r+1}$. Note that this covering provides an injection between fundamental groups, hence an embedding $\tilde{\psi}: \mathrm{B}(d, 1, r) \hookrightarrow \operatorname{Br}(r+1)$, where $\operatorname{Br}(r+1)$ denotes the usual braid group on $r+1$ strands. We choose for base point in $\mathcal{M}(r+1)$ the point $x=\left(0, x_{1}, \ldots, x_{r}\right)$ with the $x_{i}$ in $\mathbb{R}$ and $x_{i+1} \ll x_{i}$, and for generators of the usual braid group $\mathcal{M}(r+1) / \mathfrak{S}_{r+1}$ the elements $\xi_{0}, \xi_{1}, \ldots, \xi_{r-1}$ as described below:

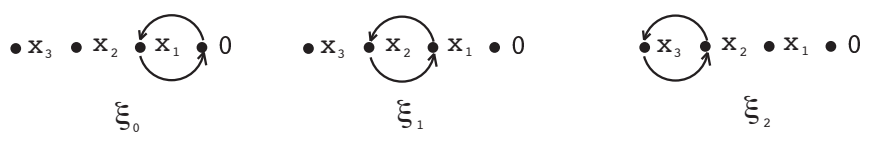

Then (see [BMR98]) the group $\pi_{1}\left(\mathcal{M}(r+1) / \mathfrak{S}_{r}\right)$ is generated by $\xi_{0}^{2}, \xi_{1}, \ldots, \xi_{r-1}$. The element $\xi_{0}^{2}$ is the class in $\mathcal{M}(r+1)$ of the loop $\left(\frac{x_{1}}{2}\left(1-e^{2 \mathrm{i} \pi t}\right), \frac{x_{1}}{2}\left(e^{2 \mathrm{i} \pi t}+1\right), x_{2}, \ldots, x_{r}\right)$. Taking its image under $p$ provides a loop based at $\left(-x_{1},-x_{2}, \ldots,-x_{r}\right)$ described by $\left(-x_{1} e^{2 \mathrm{i} \pi t}, \frac{x_{1}}{2}\left(1-e^{2 \mathrm{i} \pi t}\right)-x_{2}, \ldots, \frac{x_{1}}{2}\left(1-e^{2 \mathrm{i} \pi t}\right)-x_{r}\right)$. Since $\left|x_{i}\right| \ll\left|x_{i+1}\right|$, this path is homotopic to $\left(-x_{1} e^{2 \mathrm{i} \pi t},-x_{2}, \ldots,-x_{r}\right)$, both in $\mathcal{M}^{\#}(r)$ and in $\mathcal{M}^{\#}(r) / \mathfrak{S}_{r}$. Letting $a_{i}=-x_{i}$, we have $0<a_{1}<a_{2}<\cdots<a_{r}$, and we choose $y=\widetilde{p(x)}$ to be $y=\left(a_{1}^{1 / d}, \ldots, a_{r}^{1 / d}\right)$. The above loop thus lifts under $r$ to the path $\left(a_{1} e^{2 \mathrm{i} \pi t / d}, a_{2}, \ldots, a_{r}\right)$ in $\mathcal{M}^{\#}(d, r)$. By definition of $\psi$, the class of this path is $\sigma=\psi^{-1}\left(\xi_{0}^{2}\right)$. Similarly, we can determine $\psi^{-1}\left(\xi_{i}\right)$ when $i \geq 1$ : the image of $\xi_{i}$ under $p$ is a path in $\mathcal{M}^{\#}(r)$ homotopic to 


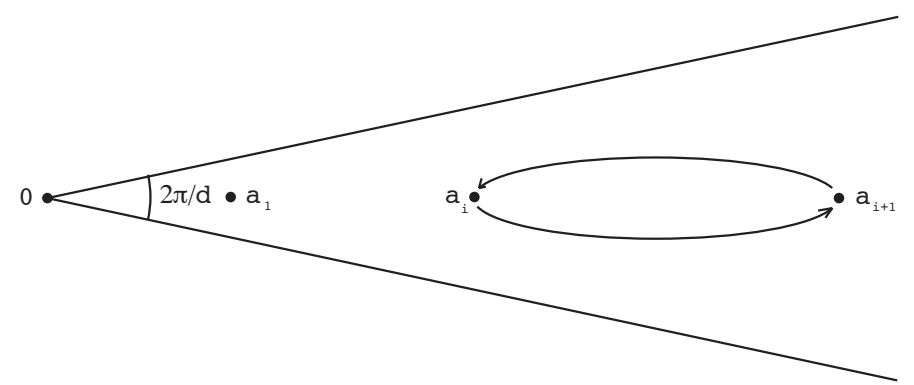

On the open cone described in the picture, the map $z \mapsto z^{d}$ is a positive homeomorphism, and this enables one to lift this path to

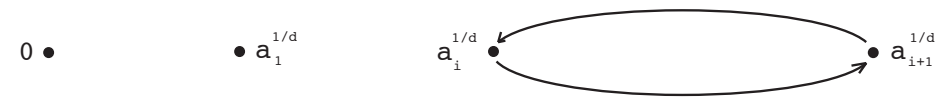

meaning that $\tau_{i}=\psi^{-1}\left(\xi_{i}\right)$ is the class of this path, from $\left(a_{1}^{1 / d}, \ldots, a_{i}^{1 / d}, a_{i+1}^{1 / d}, \ldots, a_{r}^{1 / d}\right)$ to $\left(a_{1}^{1 / d}, \ldots, a_{i+1}^{1 / d}, a_{i}^{1 / d}, \ldots, a_{r}^{1 / d}\right)$. We recall that $\mathrm{B}(d e, e, r)$ is defined to be the group $\pi_{1}\left(\mathcal{M}^{\#}(d e, r) / G(d e, e, r)\right)$ when $d>1$. If moreover $e=1$, then $\mathrm{B}(d, 1, r)$ is generated by $\sigma, \tau_{1}, \ldots, \tau_{r-1}$; in general, it is generated by $\sigma^{e}, \tau_{1}, \ldots, \tau_{r-1}$. Now, the morphism $\tilde{\psi}$ : $\mathrm{B}(d, 1, r) \hookrightarrow \operatorname{Br}(r+1)=\pi_{1}\left(\mathcal{M}(r+1) / \mathfrak{S}_{r+1}\right)$ commutes with the natural morphisms

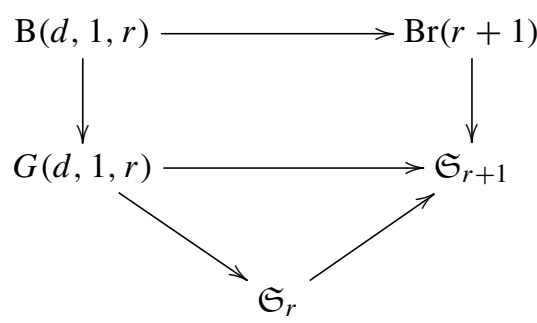

Letting as in [BMR98] $\xi_{1}^{\prime}=\xi_{0}^{2} \xi_{1} \xi_{0}^{-2} \in \pi_{1}\left(\mathcal{M}(r+1) / \mathfrak{S}_{r}, x\right)$, we have $\tau_{1}^{\prime}=\psi^{-1}\left(\xi_{1}^{\prime}\right) \in$ $\pi_{1}\left(\mathcal{M}^{\#}(d, r) / G(d, 1, r), y\right)$. As before we let $\zeta=\exp (2 \mathrm{i} \pi / d)$ and $g_{i} \in G(d, 1, r)$ being defined by $g_{i} \cdot\left(z_{1}, \ldots, z_{r}\right)=\left(z_{1}, z_{2}, \ldots, \zeta z_{i}, \ldots, z_{r}\right)$. We let $b_{i}=a_{i}^{1 / d}$.

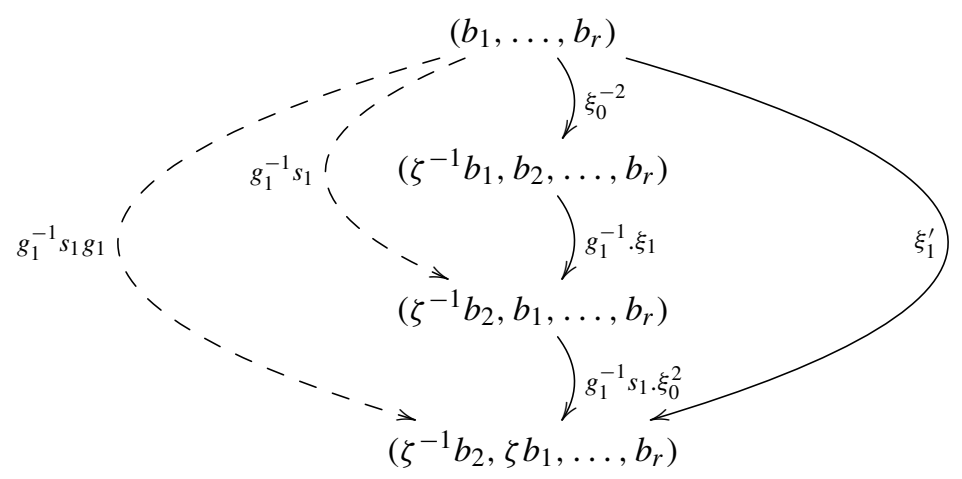


In order to generate $\mathrm{B}(e, e, r)=\pi_{1}\left(\mathcal{M}(e, r) / \mathfrak{S}_{r}, x\right)$, and letting $e=d$, we only need to take the image of $\tau_{1}^{\prime}, \tau_{1}, \ldots, \tau_{r-1}$ under $i^{*}$ where $i: \mathcal{M}^{\#}(e, r) \rightarrow \mathcal{M}(e, r)$ is the natural inclusion. We will use the following definition.

Definition 6.1. Let $X$ be the complement of a hyperplane arrangement $\mathcal{A}$ in $\mathbb{C}^{l}$, and $v, v^{\prime} \in X$. A line segment from $v$ to $v^{\prime}$ is $t \mapsto(1-t) v+t v^{\prime}$ for $t \in[0,1]$. If this line segment crosses exactly one hyperplane of $\mathcal{A}$ at one point, a positive detour from $v$ to $v^{\prime}$ is a path of the form $\gamma(t)=(1-t) v+t v^{\prime}+\mathrm{i} t(1-t)\left(v-v^{\prime}\right) \epsilon$ for $\epsilon>0$ small enough so that it and similar paths $\gamma^{\prime}$ for $0<\epsilon^{\prime}<\epsilon$ do not cross any hyperplane in $\mathcal{A}$. All such detours are clearly homotopic to each other. A negative detour is defined similarly with $\mathrm{i}$ replaced by $-\mathrm{i}$.

Note that, for $v \in \mathcal{M}(e, r)$ and $s$ a reflection in $G(e, e, r)$, if there exists a positive detour from the base point $\underline{b}=\left(b_{1}, \ldots, b_{r}\right)$ to $w \cdot \underline{b}$, then it provides a braided reflection in the hyperplane attached to $s$.

The elements $i^{*}\left(\tau_{k}\right)$ are now easy-to-describe braided reflections, as the positive detours from $\underline{b}$ to their images under the corresponding reflections. In case $e=2$, the given monoid is then clearly the classical Artin monoid of type $D_{r}$, so we can assume $e \geq 3$. The paths corresponding to $\xi_{0}^{2}$ and to its translates are homotopic to a line segment in $\mathcal{M}(e, r)$. The fact that $\tau_{1}^{\prime}$ is a braided reflection essentially amounts to the fact that $i^{*}\left(g_{0}^{-1} \cdot \tau_{1}\right)$ is a braided reflection in $\pi_{1}\left(\mathcal{M}(e, r) / G(e, e, r), g_{0}^{-1} \cdot y\right)$, and this holds true because $\tau_{1}$ is a braided reflection in $\mathcal{M}^{\#}(e, r) / G(e, e, r)$.

We consider the plane $P$ defined by the equations $z_{i}=b_{i}$ for $i=3, \ldots, r$, and identify it with $\mathbb{C}^{2}$ through $\left(z_{1}, z_{2}\right)$. We let $P^{0}=\mathbb{C}^{2} \backslash \bigcup\left\{z_{2}=z_{1} \eta \mid \eta \in \mu_{e}\right\}=$ $P \cap \mathcal{M}(e, r)$. Then $\tau_{1}, \tau_{1}^{\prime}$ lie in the plane $P$, and $\tau_{1}^{\prime}$ is homotopic in $P^{0}$ to

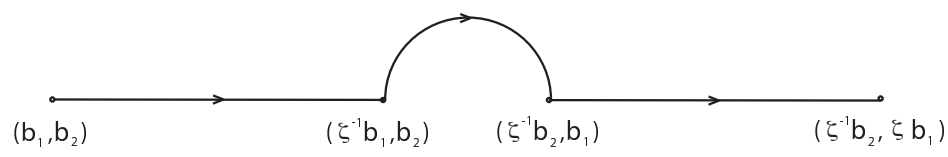

where the half-circle represents the positive detour from $\left(\zeta^{-1} b_{1}, b_{2}\right)$ to $\left(\zeta^{-1} b_{2}, b_{1}\right)$. We let now $t_{0}=\tau_{1}, t_{1}=\tau_{1}^{\prime}, t_{i+1}=t_{i}^{-1} t_{i-1} t_{i}$ for $1 \leq i \leq e-2$. A way to understand paths in $P \simeq \mathbb{C}^{2}$ is to use the projection $\mathbb{C}^{2} \rightarrow \mathbb{P}^{1}(\mathbb{C})$ given by $\left(z_{1}, z_{2}\right) \mapsto z_{2} / z_{1}$. Note for example that two paths $\gamma_{1}, \gamma_{2}$ in $P$ with the same endpoints whose images are homotopic in $\mathbb{P}^{1}(\mathbb{C}) \backslash \mu_{e}$ are homotopic in $P^{0}$ as soon as, writing $\gamma_{i}(t)=\left(x_{i}(t), y_{i}(t)\right)$, the set $x_{1}([0,1]) \cup x_{2}([0,1])$ is contained in some simply connected subspace of $\mathbb{C} \backslash\{0\}$. We let $\alpha=b_{2} / b_{1} \gg 1$. Then the positive detour $t_{0}$ is mapped to a path from $\alpha$ to $\alpha^{-1}$ close to the line segment, with image in the positive half-plane. The line segments of the form $\gamma(t)=\left(z_{1}, z_{2}(t)\right)$ are mapped to line segments, and lines $\gamma(t)=\left(z_{1}(t), z_{2}\right)$ are mapped to images of a line under $z \mapsto 1 / z$, which is the composite of the complex conjugation with the geometric inversion with respect to the unit circle; they are thus mapped to a line if the original line passes through 0 , and otherwise to a circle passing through the origin. The induced action of $G(e, e, r)$ is given by $s_{1}: z \mapsto 1 / z, g_{1}: z \mapsto \zeta^{-1} z, g_{2}: z \mapsto \zeta z$. The images of $t_{1}$ and $t_{2}$ are depicted in Figure 1. The images of $t_{2}$ and of the positive 

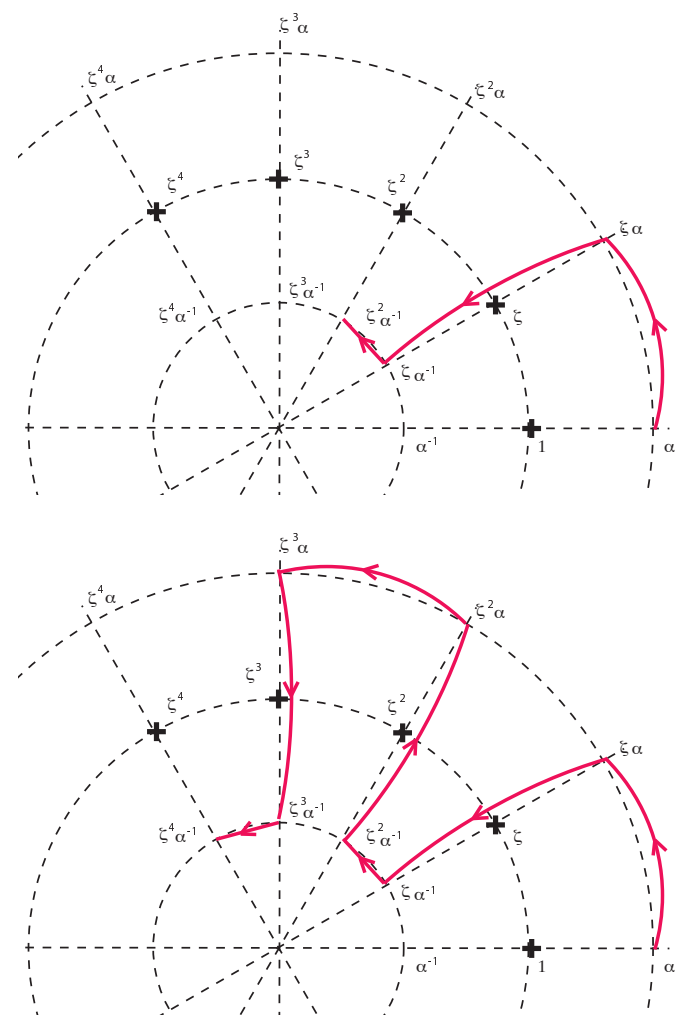

Fig. 1. Images of $t_{1}$ and $t_{2}$ in $\mathbb{P}_{1}(\mathbb{C})$.

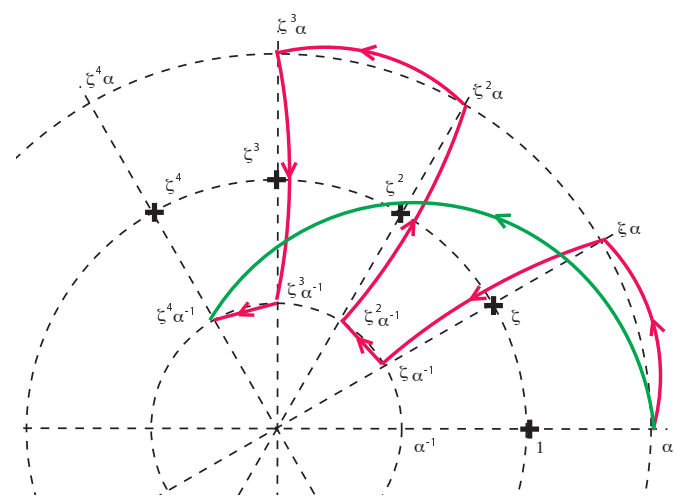

Fig. 2. $t_{2}$ and the positive detour in $\mathbb{P}_{1}(\mathbb{C})$.

detour from $\left(b_{1}, b_{2}\right)$ to $\left(\zeta^{-2} b_{2}, \zeta^{2} b_{1}\right)$ are then clearly homotopic (see Figure 2), and the first coordinate of both paths is easily checked to remain in a simply connected region of $\mathbb{C} \backslash\{0\}$. With the same argument, using the relation $t_{i+1}=t_{i}^{-1} t_{0} t_{1}$ and possibly using 


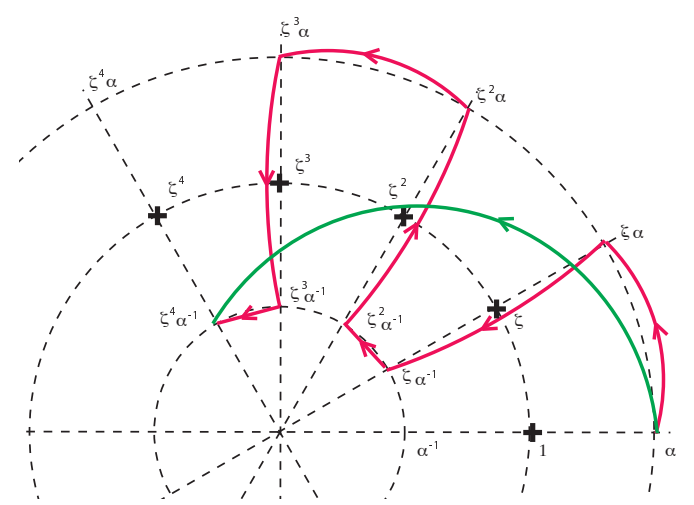

Fig. 3. Comparison between $t_{1} t_{0} t_{1}^{-1}$ and the positive detour in $\mathbb{P}_{1}(\mathbb{C})$.

$\left(z_{1}, z_{2}\right) \mapsto z_{1} / z_{2}$ instead of $\left(z_{1}, z_{2}\right) \mapsto z_{2} / z_{1}$, we conclude that each $t_{i}$ is (homotopic to) the positive detour from $\left(b_{1}, b_{2}\right)$ to $\left(\zeta^{-i} b_{2}, \zeta^{i} b_{1}\right)$. We thus get the following

Proposition 6.2. Let $\underline{b}=\left(b_{1}, \ldots, b_{r}\right) \in \mathcal{M}(e, r)$ with $0<b_{1} \ll b_{2} \ll \cdots \ll b_{r}$. Then $\mathrm{B}(e, e, r)=\pi_{1}(\mathcal{M}(e, r) / G(e, e, r), \underline{b})$ is generated by braided reflections $t_{0}, \ldots, t_{e-1}, s_{3}, \ldots, s_{r}$ which are positive detours from $\underline{b}$ to their images under the corresponding reflection. Under $\mathrm{B}(e, e, r) \rightarrow G(e, e, r), t_{i}$ is mapped to $\left(z_{1}, z_{2}, \ldots, z_{r}\right) \mapsto$ $\left(\zeta^{-i} z_{2}, \zeta^{i} z_{1}, \ldots, z_{r}\right)$, and $t_{0}, s_{3}, \ldots, s_{r}$ are mapped to the successive transpositions of $\mathfrak{S}_{r}$ in that order. These generators provide a presentation of $\mathrm{B}(e, e, r)$ with the relations (2)-(5) of page 146, and with (1) replaced by $t_{i} t_{i+1}=t_{j} t_{j+1}$.

We notice that the slight change in the presentation is meaningless in monoid-theoretic terms, as both monoids are isomorphic under $t_{i} \mapsto t_{-i}$, but it is not in topological terms, as $t_{1} t_{0} t_{1}^{-1}$ is not homotopic to a detour from $\underline{b}$ to its image (see Figure 3 ).

Proposition 6.3. Let $S_{0}=\left\{t_{i}, s_{3}, \ldots, s_{r}\right\}$. Than the subgroup of $\mathrm{B}(e, e, r)=$ $\pi_{1}(\mathcal{M}(e, r) / G(e, e, r))$ generated by $S_{0}$ is a parabolic subgroup in the sense of [BMR98], and can be naturally identified with the braid group on $r$ strands as the fundamental group of $\left\{\left(z_{1}, \ldots, z_{r}\right) \mid z_{i} \neq z_{j}, z_{1}+\cdots+z_{r}=0\right\} / \mathfrak{S}_{r}$, with base point $\left(-\zeta^{-i}\left(b_{1}+b_{2}+\cdots+b_{r}\right), b_{2}, \ldots, b_{r}\right)$, in such a way that the elements of $S_{0}$ are identified with positive detours.

Proof. The parabolic subgroup of $G(e, e, r)$ defined as the fixer of $\left(\zeta^{i}, 1,1, \ldots, 1\right)$ is obviously conjugate to the one fixing $(1,1, \ldots, 1)$, the latter being the natural $\mathfrak{S}_{r} \subset$ $G(e, e, r)$. We thus need only consider the case $i=0$. Let $\alpha=-\left(b_{1}+\cdots+b_{r}\right) \ll 0$, $\underline{b}_{0}=\left(\alpha, b_{2}, \ldots, b_{r}\right), X=\mathcal{M}(e, r)$ and $X_{0}=\left\{\left(z_{1}, \ldots, z_{r}\right) \mid z_{i} \neq z_{j}, z_{1}+\cdots+z_{r}=0\right\}$. By [BMR98] we get an embedding $\pi_{1}\left(X_{0} / \mathfrak{S}_{r}, \underline{b}_{0}\right) \hookrightarrow \pi_{1}(X / G(e, e, r), \underline{b})$, natural only up to the choice of a path from $\underline{b}$ to $\underline{b}_{0}$ in $Y$. The line segment $\gamma$ from $\underline{b}$ to $\underline{b}_{0}$ provides such a natural choice.

We now need to prove that composing the positive detours from $\underline{b}$ with this path provides the positive detours from $\underline{b}_{0}$, up to homotopy in $Y=\left\{\left(z_{1}, \ldots, z_{r}\right) \mid z_{i} \neq z_{j}\right\}$. 
For $s_{3}, \ldots, s_{r}$ this is true because the first component of the first path can be homotoped to the second one in $\left(\mathbb{R}_{\leq b_{1}}, b_{1}\right)$. For $t_{0}$ we let $\sigma_{0}$ and $\sigma$ denote the positive detours in $P^{0}$ from $\left(b_{1}, b_{2}\right)$ to $\left(b_{2}, b_{1}\right)$ and from $\left(\alpha, b_{2}\right)$ to $\left(b_{2}, \alpha\right)$, respectively. Let $\gamma, \gamma^{\prime}$ denote the line segments $\left(b_{1}, b_{2}\right) \rightarrow\left(\alpha, b_{2}\right)$ and $\left(b_{2}, \alpha\right) \rightarrow\left(b_{2}, b_{1}\right)$. We need to prove that $\sigma_{0}$ is homotopic to $\gamma^{\prime} \sigma \gamma$ in $\left\{\left(z_{1}, z_{2}\right) \in \mathbb{C}^{2} \mid z_{1} \neq z_{2}\right\}$, the other coordinates $z_{3}, \ldots, z_{r}$ being the same for both paths. Since $b_{1}-b_{2}$ and $\alpha-b_{2}$ have the same (negative) sign, we can homotope $\gamma^{\prime} \sigma \gamma$ to a path with the same real part (for both coordinates), and with imaginary part the same as $\sigma$, up to possibly diminishing the chosen factor $\epsilon$ in the definition of the detours. Choosing then a homotopy in $\mathbb{R}_{\leq b_{2}}^{2}$ between the real parts of these two paths provides a homotopy between them in $Y$.

\subsection{Parabolic submonoids}

We apply the results of Section 5.4 on submonoids to the monoid $N=M(e, e, r)$, with generators $S=\left\{t_{0}, \ldots, t_{e-1}, s_{3}, \ldots, s_{r}\right\}$. Let $\mathcal{C}=\left\{t_{0}, \ldots, t_{e-1}\right\}$. For $S_{0} \subset S$, let $M\left(S_{0}\right)$ be the monoid generated by $S_{0}$ with the defining relations of $M(e, e, r)$ which involve only elements of $S_{0}$. We get a natural morphism $\varphi: M\left(S_{0}\right) \rightarrow M(S)=M(e, e, r)$. We make the following extra assumption on $S_{0}$ :

$$
S_{0} \cap \mathcal{C} \in\left\{\emptyset, \mathcal{C},\left\{t_{i}\right\}\right\} \quad \text { for some } i \in\{0,1, \ldots, e-1\} .
$$

In other terms, $S_{0}$ contains none, all or exactly one of the $t_{i}$ 's. Note that all the corresponding monoids are known to be Garside and are endowed with a suitable length function.

This condition implies the extra condition on $\varphi$ in Section 5.4, namely that if $n$ in $M(S)$ divides $\varphi(m)$ for some $m \in M\left(S_{0}\right)$, then $n \in \varphi\left(M\left(S_{0}\right)\right)$. Indeed, if we have such $n, m$, then $n \in \varphi\left(M\left(S_{0}\right)\right)$ unless $n$ can be written as a word containing some $x \in S \backslash S_{0}$. But in that case $\varphi(m)$ can also be written as a word in $S$ containing $x$. Now note that the defining relations involving such an $x$ cannot make it disappear, except when $x \in \mathcal{C}$. By contradiction this settles the cases $S_{0} \cap \mathcal{C}=\emptyset$ and $S_{0} \supset \mathcal{C}$. In case $S_{0} \cap \mathcal{C}=\left\{t_{i}\right\}$, we can assume $x=t_{j}$ for $j \neq i$, and would get equality in $M(S)$ of two words on $S$, one involving $t_{i}$ and no other element of $\mathcal{C}$, and the other involving $t_{j}$. But we check on the defining relations that all relations involving $t_{i}$ either involve only $t_{i}$ and no other elements of $\mathcal{C}$ in which case they preserve that property and do not make the $t_{i}$ 's disappear, or they involve several elements of $\mathcal{C}$ and cannot be applied to the first word. This leads to a contradiction, which proves this property.

This condition also implies the property (2) for $\varphi$. For this we need to compute the lcm's of two elements $x, y$ of $S$. We need to prove the following in $M\left(S_{0}\right)$, for any $S_{0} \subset S$ satisfying the above condition that contains $x$ and $y$ :

- $\operatorname{lcm}\left(s_{i}, s_{j}\right)=s_{i} s_{j}=s_{j} s_{i}$ if $|j-i| \geq 2$,

- $\operatorname{lcm}\left(s_{i}, s_{i+1}\right)=s_{i} s_{i+1} s_{i}=s_{i+1} s_{i} s_{i+1}$,

- $\operatorname{lcm}\left(t_{i}, t_{j}\right)=t_{1} t_{0}=t_{i} t_{i-1}=t_{j} t_{j-1}$,

- $\operatorname{lcm}\left(t_{i}, s_{3}\right)=t_{i} s_{3} t_{i}=s_{3} t_{i} s_{3}$,

- $\operatorname{lcm}\left(t_{i}, s_{j}\right)=t_{i} s_{j}=s_{j} t_{i}$ if $j \geq 4$. 
The identities with length two are clear, as the lcm exist and cannot have length 1 . For the ones of length 3 , namely $\{x, y\}=\left\{s_{i}, s_{i+1}\right\}$ and $\{x, y\}=\left\{t_{i}, s_{3}\right\}$, we use that $\{x, y\} \subset$ $S_{0} \subset S$ satisfies our condition. Since the lcm of $x, y$ in $M\left(S_{0}\right)$ should divide $x y x=y x y$, it should then come from $M(\{x, y\})$, meaning that it should be a word in $x$ and $y$, of length at most 2 . Thus only few possibilities remain, all of them easily excluded.

Using the previous section, we thus get injective monoid morphisms $M\left(S_{0}\right) \rightarrow M(S)$ $=M(e, e, r)$. Let $B\left(S_{0}\right)$ be the group of fractions of $M\left(S_{0}\right)$. It is proved in [CP] that $B(S)=\mathrm{B}(e, e, r)$. We call the $B\left(S_{0}\right)$ the parabolic submonoids of $\mathrm{B}(e, e, r)$. Crucial examples of such submonoids are described below.

6.3.1. Second homology group. We order the atoms so that $s_{r}<s_{r-1}<\cdots<s_{3}<t_{0}<$ $t_{1}<\cdots<t_{e-1}$. By the above construction, the parabolic submonoid $M(e, e, r-1)=$ $M\left(\left\{s_{r-1}, \ldots, s_{3}, t_{0}, \ldots, t_{e-1}\right\}\right)$ is indeed a submonoid of $M(e, e, r)$, and the $1 \mathrm{~cm}$ of a family of elements in $M(e, e, r-1)$ is also its lcm in $M(e, e, r)$. The same holds true for the following submonoids:

- generated by $s_{3}, t_{i}$, which is an Artin monoid of type $A_{2}$;

- generated by $s_{k}, t_{i}, k \geq 4$, which is an Artin monoid of type $A_{1} \times A_{1}$;

- generated by $s_{4}, s_{3}, t_{i}$, which is an Artin monoid of type $A_{3}$;

- generated by $s_{k}, s_{3}, t_{i}, k \geq 5$, which is an Artin monoid of type $A_{1} \times A_{2}$;

- generated by $s_{k}, s_{l}, t_{i}, k \geq l+2, l \geq 4$, which is an Artin monoid of type $A_{1} \times A_{1} \times A_{1}$;

- generated by $s_{k}, s_{l}, s_{r}$, which is an Artin monoid of the type given by the obvious subdiagram (of type $A_{1} \times A_{1} \times A_{1}, A_{2} \times A_{1}, A_{1} \times A_{2}$ or $A_{3}$ ).

We first compute the differentials of the top cell for the corresponding Artin monoids (see Table 8), and then use this remark computing the differentials of the 2-cells and 3cells. We let $d_{n}=\partial_{n} \otimes_{\mathbb{Z} M} \mathbb{Z}: C_{n} \otimes_{\mathbb{Z} M} \mathbb{Z} \rightarrow C_{n-1} \otimes_{\mathbb{Z} M} \mathbb{Z}$ denote the differential with trivial coefficients.

The 2-cells are the following: $\left[t_{0}, t_{i}\right]$ for $1<i<e,\left[s_{3}, t_{i}\right],\left[s_{k}, t_{i}\right]$ for $k \geq 4$ and $\left[s_{k}, s_{l}\right]$ for $k<l$. From Table 8 we get

$$
\begin{aligned}
d_{2}\left[t_{0}, t_{i}\right] & =\left[t_{i}\right]+\left[t_{i+1}\right]-\left[t_{0}\right]-\left[t_{1}\right], \\
d_{2}\left[s_{3}, t_{i}\right] & =\left[t_{i}\right]-[s], \\
d_{2}\left[s_{k}, t_{i}\right] & =0 \quad \text { if } k \geq 4, \\
d_{2}\left[s_{k}, s_{l}\right] & =0 \quad \text { if } l>k+2 \geq 4, \\
d_{2}\left[s_{k}, s_{k+1}\right] & =\left[s_{k+1}\right]-\left[s_{k}\right] .
\end{aligned}
$$

We let $\delta_{2}=t_{1} t_{0}$ denote the Garside element of $M(e, e, 2)$ and we assume $e>1$. For 3 -cells, we also need to compute

$$
\begin{aligned}
\partial_{3}\left[s, t_{0}, t_{j}\right]= & \left(s \delta_{2} s-t_{j+2} t_{j+1} s+t_{j+2} s\right)\left[t_{0}, t_{j}\right]-t_{j+2} s t_{j+1}\left[s, t_{j}\right] \\
& +\left(t_{j+2}-s t_{j+2}\right)\left[s, t_{j+1}\right]+\left(s-t_{j+2} s-1\right)\left[t_{0}, t_{j+1}\right]+\left(s t_{2}-t_{2}\right)\left[s, t_{1}\right] \\
& +\left(t_{2} s+1-s\right)\left[t_{0}, t_{1}\right]+\left[s, t_{j+2}\right]+t_{2} s t_{1}\left[s, t_{0}\right]-\left[s, t_{2}\right]
\end{aligned}
$$


when $j \not \equiv-1 \bmod e$, and

$$
\begin{aligned}
\partial_{3}\left[s, t_{0}, t_{-1}\right]= & \left(s \delta_{2} s-t_{1} t_{0} s+t_{1} s\right)\left[t_{0}, t_{-1}\right]-t_{1} s t_{0}\left[s, t_{-1}\right]+\left(1-t_{2}+s t_{2}\right)\left[s, t_{1}\right] \\
& +\left(1+t_{2} s-s\right)\left[t_{0}, t_{1}\right]+\left(t_{1}-s t_{1}\right)\left[s, t_{0}\right]+t_{2} s t_{1}\left[s, t_{0}\right]-\left[s, t_{2}\right] .
\end{aligned}
$$

This means $d_{3}\left[s, t_{0}, t_{-1}\right]=\left[t_{0}, t_{-1}\right]-\left[s, t_{-1}\right]+\left[s, t_{1}\right]+\left[t_{0}, t_{1}\right]+\left[s, t_{0}\right]-\left[s, t_{2}\right]$ and $d_{3}\left[s, t_{0}, t_{j}\right]=\left[t_{0}, t_{j}\right]-\left[s, t_{j}\right]-\left[t_{0}, t_{j+1}\right]+\left[t_{0}, t_{1}\right]+\left[s, t_{j+2}\right]+\left[s, t_{0}\right]-\left[s, t_{2}\right]$ for $j \not \equiv$ $1 \bmod e$.

\begin{tabular}{|c|c|c|c|}
\hline Type & Atoms & Relations & Differential of top cell \\
\hline$M(e, e, 2)$ & $t_{0}<\cdots<t_{r}$ & $t_{i} t_{i+1}=t_{j} t_{j+1}$ & $\partial_{2}\left[t_{0}, t_{i}\right]=t_{i+1}\left[t_{i}\right]+\left[t_{i+1}\right]-t_{1}\left[t_{0}\right]-\left[t_{1}\right]$ \\
\hline$M(e, e, 2) \times A_{1}$ & $s<t_{0}<\cdots<t_{r}$ & $\mid \begin{aligned} t_{i} t_{i+1} & =t_{j} t_{j+1} \\
t_{i} s & =s t_{i}\end{aligned}$ & $\begin{aligned} \partial_{2}\left[s, t_{0}, t_{i},\right]= & (s-1)\left[t_{0}, t_{i}\right]-t_{i+1}\left[s, t_{i}\right] \\
& +t_{1}\left[s, t_{0}\right]-\left[s, t_{i+1}\right]+\left[s, t_{1}\right]\end{aligned}$ \\
\hline$A_{2}$ & $s<t$ & $s t s=t s t$ & $\partial_{2}[s, t]=(t s+1-s)[t]+(t-s t-1)[s]$ \\
\hline$A_{1} \times A_{1}$ & $s<u$ & $s u=u s$ & $\partial_{2}[s, u]=(s-1)[u]-(u-1)[s]$ \\
\hline$A_{3}$ & $s<t<u$ & $\begin{aligned} s t s & =t s t \\
s u & =u s \\
t u t & =u t u\end{aligned}$ & $\begin{aligned} \partial_{3}[s, t, u]= & (u+s t u-t u-1)[s, t]-[s, u] \\
& +(s u-u-s+1-t s u) t[s, u] \\
& +(s-1-t s+u t s)[t, u]\end{aligned}$ \\
\hline$A_{2} \times A_{1}$ & $s<t<u$ & $\begin{aligned} t u & =u t \\
s u & =u s \\
s t s & =t s t\end{aligned}$ & $\begin{aligned} \partial_{3}[s, t, u]= & (1-s+t s)[t, u] \\
& +(t-1-s t)[s, u] \\
& +(u-1)[s, t]\end{aligned}$ \\
\hline$A_{1} \times A_{2}$ & $s<t<u$ & $\begin{aligned} s t & =t s \\
s u & =u s \\
t u t & =u t u\end{aligned}$ & $\begin{aligned} \partial_{3}[s, t, u]= & (1+t u-u)[s, t] \\
& +(t-1-u t)[s, u] \\
& +(s-1)[t, u]\end{aligned}$ \\
\hline$A_{1} \times A_{1} \times A_{1}$ & $s<t<u$ & $\begin{aligned} s u & =u s \\
s t & =t s \\
t u & =u t\end{aligned}$ & $\begin{aligned} \partial_{3}[s, t, u]= & (1-t)[s, u] \\
& +(u-1)[s, t] \\
& +(s-1)[t, u]\end{aligned}$ \\
\hline
\end{tabular}

Table 8. Top cells for monoids of small type.

We now compute the second homology group, starting with $\operatorname{Ker} d_{2}$. Let

$$
v_{i}=\left[t_{0}, t_{i}\right]+\left[s, t_{0}\right]+\left[s, t_{1}\right]-\left[s, t_{i}\right]-\left[s, t_{i+1}\right] \in \operatorname{Ker} d_{2}
$$

for $1 \leq i \leq e-1$. Let $K_{1}$ denote the submodule of $\operatorname{Ker} d_{2}$ spanned by the $v_{i}$. It is easy to show that $K_{1}$ is free on the $v_{i}$, and $K_{1}=\operatorname{Ker} d_{2}$ for $r=3$; if $r>3$ we have $\operatorname{Ker} d_{2}=K_{1} \oplus K_{2}$ where $K_{2}$ is the free $\mathbb{Z}$-module with basis the [ $\left.s_{k}, t_{i}\right]$ for $k \geq 4$ and, if $r \geq 5$, the $\left[s_{l}, s_{k}\right]$ for $l \geq k+2, k \geq 3$.

Now decompose $\mathbb{Z} \mathcal{X}_{3}=C_{1} \oplus C_{2}$ where $C_{1}$ has for basis the $\left[s_{3}, t_{0}, t_{i}\right]$ and $C_{2}$ has for basis the other 3 -cells. By the above computations we get $d_{3}\left(C_{1}\right) \subset K_{1}$ and $d_{3}\left(C_{2}\right) \subset K_{2}$. 
Thus $H_{2}(B, \mathbb{Z})=\left(K_{1} / d_{3}\left(C_{1}\right)\right) \oplus\left(K_{2} / d_{3}\left(C_{2}\right)\right)$. We first compute $K_{1} / d_{3}\left(C_{1}\right)$. We have

$$
\begin{aligned}
d_{3}\left[s_{3}, t_{0}, t_{j}\right] & =v_{j}-v_{j+1}+v_{1} \quad \text { if } 0<j<e-1, \\
d_{3}\left[s_{3}, t_{0}, t_{e-1}\right] & =v_{e-1}+v_{1}+v_{1} .
\end{aligned}
$$

We denote $u_{i}=\left[s_{3}, t_{0}, t_{i}\right]$ for $1 \leq i \leq e-1$, and let $w_{i}=u_{i}+u_{i+1}+\cdots+u_{e-1}$. Then $d_{3} w_{i}=v_{i}+(e-i) v_{1}$. In the $\mathbb{Z}$-basis $\left(w_{i}\right)$ and $\left(v_{i}\right), d_{3}$ is in triangular form, and the only diagonal coefficient that differs from 1 is $e$, since $d_{3} w_{1}=e v_{1}$. It follows that $K_{1} / d_{3}\left(C_{1}\right) \simeq \mathbb{Z}_{e}$. Since $H_{2}(B, \mathbb{Z})=K_{1} / d_{3}\left(C_{1}\right)$ for $r=3$, we can now assume $r \geq 4$.

First assume $r=4$. In $K_{2} / d_{3}\left(C_{2}\right)$ we have $2\left[s_{4}, t_{i}\right] \equiv 0$, because $d_{3}\left[s_{4}, s_{3}, t_{i}\right]=$ $-2\left[s_{4}, t_{i}\right]$. Since $d_{3}\left[s_{4}, t_{0}, t_{i}\right]=-\left[s_{4}, t_{i}\right]+\left[s_{4}, t_{0}\right]-\left[s_{4}, t_{i+1}\right]+\left[s_{4}, t_{1}\right]$, we get $\left[s_{4}, t_{i}\right]+$ $\left[s_{4}, t_{i+1}\right] \equiv\left[s_{4}, t_{0}\right]+\left[s_{4}, t_{1}\right]$ when $i>0$. In particular, $\left[s_{4}, t_{i}\right]+\left[s_{4}, t_{i+1}\right] \equiv\left[s_{4}, t_{i+1}\right]+$ $\left[s_{4}, t_{i+2}\right]$, that is, $\left[s_{4}, t_{i}\right] \equiv\left[s_{4}, t_{i+2}\right]$, at least if $0<i<e-1$. From $d_{3}\left[s_{4}, t_{0}, t_{1}\right] \equiv 0$ we deduce $\left[s_{4}, t_{2}\right] \equiv\left[s_{4}, t_{0}\right]$, and from $d_{3}\left[s_{4}, t_{0}, t_{e-1}\right] \equiv 0$ we deduce $\left[s_{4}, t_{e-1}\right] \equiv\left[s_{4}, t_{1}\right]$. Thus $\left[s_{4}, t_{i}\right] \equiv\left[s_{4}, t_{i+2}\right]$ for every $i$. When $e$ is odd, $K_{2} / d_{3}\left(C_{3}\right)$ is spanned by the class of $\left[s_{4}, t_{0}\right]$. From the other relations one easily sees that this class is non-zero, and since $2\left[s_{4}, t_{i}\right] \equiv 0$ we get $K_{2} / d_{3}\left(C_{2}\right) \simeq \mathbb{Z}_{2}$. When $e$ is even, this quotient is spanned by the classes of $\left[s_{4}, t_{0}\right]$ and $\left[s_{4}, t_{1}\right]$, and we get similarly $K_{2} / d_{3}\left(C_{2}\right) \simeq \mathbb{Z}_{2}^{2}$.

We now assume $r \geq 5$. Then $d_{3}\left(\left[s_{5}, s_{3}, t_{i}\right]\right)=\left[s_{5}, s_{3}\right]-\left[s_{5}, t_{i}\right]$ whence $a:=\left[s_{5}, t_{0}\right] \equiv$ $\left[s_{5}, t_{i}\right]$ for all $i$, regardless whether $e$ is even or odd. From $d_{3}\left[s_{5}, s_{4}, t_{i}\right]=\left[s_{4}, t_{i}\right]-\left[s_{5}, t_{i}\right]$ we get $\left[s_{4}, t_{i}\right] \equiv a$ and from $\left[s_{k+1}, s_{k}, t_{i}\right]=\left[s_{k}, t_{i}\right]-\left[s_{k+1}, t_{i}\right]$ we deduce by induction $\left[s_{k}, t_{i}\right] \equiv a$. The only remaining relation involving $a$ is then as before $2 a \equiv 0$.

On the other hand, we have $\left[s_{5}, s_{3}\right] \equiv a$. Assume we have $\left[s_{l}, s_{k}\right] \equiv a$ for some $l, k$ with $l \geq k+2$. From $d_{3}\left[s_{l}, s_{k+1}, s_{k}\right]=\left[s_{l}, s_{k+1}\right]-\left[s_{l}, s_{k}\right]$ for $l \geq k+3$ we get $\left[s_{l}, s_{k^{\prime}}\right] \equiv a$ for all $k^{\prime}$ with $l-2 \geq k^{\prime} \geq 3$, and therefore $\left[s_{l^{\prime}}, s_{k^{\prime}}\right] \equiv a$ for all $l^{\prime}, k^{\prime}$ with $l^{\prime}-2 \geq k^{\prime} \geq 3$. We thus get $K_{2} / d_{3} C_{2} \simeq \mathbb{Z} / 2 \mathbb{Z}$.

As a consequence, we get the following result.

Theorem 6.4. Let $B=\mathrm{B}(e, e, r)$ with $r \geq 3$ and $e \geq 2$.

- When $r=3, H_{2}(B, \mathbb{Z}) \simeq \mathbb{Z}_{e}$.

- When $r=4$ and $e$ is odd, $H_{2}(B, \mathbb{Z}) \simeq \mathbb{Z}_{e} \times \mathbb{Z}_{2} \simeq \mathbb{Z}_{2 e}$.

- When $r=4$ and e is even, $H_{2}(B, \mathbb{Z}) \simeq \mathbb{Z}_{e} \times \mathbb{Z}_{2}^{2}$.

- When $r \geq 5, H_{2}(B, \mathbb{Z}) \simeq \mathbb{Z}_{e} \times \mathbb{Z}_{2}$.

The case $r=2$ is when $W$ is a dihedral group, and this case is known by [Sal94]: we have $H_{2}(B, \mathbb{Z})=0$ if $e$ is odd, $H_{2}(B, \mathbb{Z})=\mathbb{Z}$ if $e$ is even.

\section{Low-dimensional homology}

\subsection{The second homology group}

The computations above provide the second integral homology group $H_{2}(B, \mathbb{Z})$. In the case of the finite group $W$, the group $\mathrm{H}_{2}(W, \mathbb{Z})$ can be identified with the Schur multiplier $H^{2}\left(W, \mathbb{C}^{\times}\right)$, which is relevant for dealing with projective representations. We use the determination of the $H_{2}(B, \mathbb{Z})$ to show a direct connection between the two groups $H^{2}\left(B, \mathbb{C}^{\times}\right)$and $H^{2}\left(W, \mathbb{C}^{\times}\right)$. We start with a lemma. 
Lemma 7.1. Let $W$ be an irreducible finite complex 2-reflection group, and $B$ the associated braid group. The inflation morphism $H^{2}\left(W, \mathbb{C}^{\times}\right) \rightarrow H^{2}\left(B, \mathbb{C}^{\times}\right)$is injective.

Proof. The Hochschild-Serre exact sequence associated to $1 \rightarrow P \rightarrow B \rightarrow W \rightarrow 1$ is

$$
0 \rightarrow H^{1}\left(W, \mathbb{C}^{\times}\right) \rightarrow H^{1}\left(B, \mathbb{C}^{\times}\right) \rightarrow H^{1}\left(P, \mathbb{C}^{\times}\right)^{W} \rightarrow H^{2}\left(W, \mathbb{C}^{\times}\right) \rightarrow H^{2}\left(B, \mathbb{C}^{\times}\right) .
$$

Now $H^{1}\left(P, \mathbb{C}^{\times}\right)=\operatorname{Hom}\left(P^{\mathrm{ab}}, \mathbb{C}^{\times}\right)^{W}=\operatorname{Hom}\left(\left(P^{\mathrm{ab}}\right)^{W}, \mathbb{C}^{\times}\right)$and $H^{1}\left(B, \mathbb{C}^{\times}\right)=$ $\operatorname{Hom}\left(B^{\mathrm{ab}}, \mathbb{C}^{\times}\right)$. Now $P^{\mathrm{ab}}=H_{1}(P, \mathbb{Z})$ and $B^{\mathrm{ab}}$ (see [BMR98, Thm. 2.17]) are torsionfree, with $B^{\mathrm{ab}} \simeq \mathbb{Z}^{r}$ where $r$ denotes the number of hyperplane orbits, and $\left(P^{\mathrm{ab}}\right)^{W}$ can be identified with $(2 \mathbb{Z})^{r}$. The induced map $\operatorname{Hom}\left(\mathbb{Z}^{r}, \mathbb{C}^{\times}\right) \rightarrow \operatorname{Hom}\left((2 \mathbb{Z})^{r}, \mathbb{C}^{\times}\right)$is then onto, since $\mathbb{C}$ is algebraically closed. By the Hochschild-Serre exact sequence above the conclusion follows.

Remark 7.2. Another proof of the lemma can be given using projective representations instead of the Hochschild-Serre exact sequence. Let $\alpha \in Z^{2}\left(W, \mathbb{C}^{\times}\right)$with zero image in $H^{2}\left(B, \mathbb{C}^{\times}\right)$, choose some projective representation $R$ of $W$ with 2-cocycle $\alpha$, and consider its lift $\tilde{R}$ to $B$. By assumption, it is linearizable to some linear representation $\tilde{S}$. Choosing one generator of the monodromy $\sigma_{i}$ in $X / W$ for each hyperplane orbit (see [BMR98, Appendix A]) we find that $\tilde{S}\left(\sigma_{i}^{2}\right)=\lambda_{i} \in \mathbb{C}^{\times}$. By [BMR98, Theorem 2.17] there exists a morphism $\varphi: B \rightarrow \mathbb{C}^{\times}$with $\varphi\left(\sigma_{i}\right)=1 / \lambda_{i}$, and then $\tilde{T}=\tilde{S} \circ \varphi$ is a linear representation of $B$ that factors through $W$ and linearizes $R$, thus proving that $\alpha$ has zero image in $H^{2}\left(W, \mathbb{C}^{\times}\right)$.

It is known by work of Read [Rea76] and van der Hout [vdH77] that $H^{2}\left(W, \mathbb{C}^{\times}\right) \simeq$ $H_{2}(W, \mathbb{Z})$ is a free $\mathbb{Z}_{2}$-module in all cases. A nice property that follows from our computation is that the part of $H^{2}\left(B, \mathbb{C}^{\times}\right)$that comes from $H^{2}\left(W, \mathbb{C}^{\times}\right)$is exactly the 2-torsion (except for two exceptional cases). Indeed, since $H_{1}(B, \mathbb{Z})$ is torsion-free and $\mathbb{C}^{\times}$is divisible, by the Universal Coefficients Theorem we get $H^{2}\left(B, \mathbb{C}^{\times}\right) \simeq \operatorname{Hom}\left(H_{2} B, \mathbb{C}^{\times}\right)$and the proposition below is a consequence of our computation of $H_{2}(B, \mathbb{Z}$ ) (see Table 10 for the exceptional groups, Theorems 1.4 and 6.4 for the $G(2 e, e, r)$ and the $G(e, e, r))$ and of the work of Read and van der Hout on $W$. We recall their computation of $H_{2}(W, \mathbb{Z})$ in Table 10 for the exceptional groups, and the rank over $\mathbb{Z}_{2}$ for the other ones in Table 9 .

Table 9. Rank of $H_{2}(W, \mathbb{Z})$ as a $\mathbb{Z}_{2}$-module (after Reid).

\begin{tabular}{|c|l|c|c|}
\hline$r$ & $e$ & $G(e, e, r)$ & $G(2 e, e, r)$ \\
\hline 2 & odd & 0 & 1 \\
& even & 1 & 2 \\
\hline 3 & odd & 0 & 2 \\
& even & 1 & 2 \\
\hline 4 & odd & 1 & 3 \\
& even & 3 & 4 \\
\hline 5 & odd & 1 & 3 \\
& even & 2 & 3 \\
\hline
\end{tabular}


Table 10. The second integral homology groups.

\begin{tabular}{|c|c|c||c|c|c|}
\hline$W$ & $H_{2} W$ & $H_{2} B$ & $W$ & $H_{2} W$ & $H_{2} B$ \\
\hline$G_{12}$ & 0 & 0 & $G_{30}$ & $\mathbb{Z}_{2}$ & $\mathbb{Z}_{2}$ \\
$G_{13}$ & $\mathbb{Z}_{2}$ & $\mathbb{Z}$ & $G_{31}$ & $\mathbb{Z}_{2}$ & $\mathbb{Z}_{6}$ \\
$G_{22}$ & 0 & 0 & $G_{33}$ & 0 & $\mathbb{Z}_{6}$ \\
$G_{23}$ & $\mathbb{Z}_{2}$ & $\mathbb{Z}$ & $G_{34}$ & 0 & $\mathbb{Z}_{6}$ \\
$G_{24}$ & $\mathbb{Z}_{2}$ & $\mathbb{Z}$ & $G_{35}$ & $\mathbb{Z}_{2}$ & $\mathbb{Z}_{2}$ \\
$G_{27}$ & $\mathbb{Z}_{2}$ & $\mathbb{Z}_{3} \times \mathbb{Z}$ & $G_{36}$ & $\mathbb{Z}_{2}$ & $\mathbb{Z}_{2}$ \\
$G_{28}$ & $\left(\mathbb{Z}_{2}\right)^{2}$ & $\mathbb{Z}^{2}$ & $G_{37}$ & $\mathbb{Z}_{2}$ & $\mathbb{Z}_{2}$ \\
$G_{29}$ & $\left(\mathbb{Z}_{2}\right)^{2}$ & $\mathbb{Z}_{2} \times \mathbb{Z}_{4}$ & & & \\
\hline
\end{tabular}

Proposition 7.3. Except for $W=G_{33}$ and $W=G_{34}, H^{2}\left(W, \mathbb{C}^{\times}\right)$coincides with the 2-torsion of $H^{2}\left(B, \mathbb{C}^{\times}\right)$.

\subsection{First homology in the sign representation}

If $r=|\mathcal{A} / W|$ denotes the number of hyperplane classes, the abelianization $B_{\mathrm{ab}}$ is isomorphic to $\mathbb{Z}^{r}$. There are thus $2^{r}-1$ non-zero morphisms $B \rightarrow \mathbb{Z}_{2}$, which define $2^{r}-1$ subgroups of even braids. When $r=1$, there is only one such morphism $\epsilon: B \rightarrow \mathbb{Z}_{2}$ and group $B^{(2)}=\operatorname{Ker} \epsilon$. We investigate here two abelian invariants of $B$ which are naturally attached to this group: the abelianization $B_{\mathrm{ab}}^{(2)}$ of $B^{(2)}$ and $H_{1}\left(B, \mathbb{Z}_{\epsilon}\right)$.

Lemma 7.4. Let $u \in B \backslash B^{(2)}$. The group $H_{1}\left(B, \mathbb{Z}_{\epsilon}\right)$ is isomorphic to the quotient of $B_{\mathrm{ab}}^{(2)}$ by the relations $\left[u^{2}\right] \equiv 0$ and $\left[h^{u}\right] \equiv-[h]$ for $h \in B_{\mathrm{ab}}^{(2)}$, where $h^{u}=u^{-1} h u$.

Proof. We start from the bar resolution $C_{2} \rightarrow C_{1} \rightarrow C_{0}$, where $C_{i}$ is a free $\mathbb{Z} B$-module with basis the $\left[g_{1}, \ldots, g_{i}\right]$ for $g_{i} \in B$, and we have $d_{1}([g])=(g-1)[\emptyset], d_{2}\left(\left[g_{1}, g_{2}\right]\right)=$ $g_{1}\left[g_{2}\right]-\left[g_{1} g_{2}\right]+\left[g_{1}\right]$. Denoting by $d_{i}^{\epsilon}$ the differential with coefficients in $\mathbb{Z}_{\epsilon}$ and $C_{i}^{\epsilon}=$ $C_{i} \otimes_{\mathbb{Z} B} \mathbb{Z}_{\epsilon}$ with $\mathbb{Z}$-basis the $\left[g_{1}, \ldots, g_{i}\right]$, we find that $\operatorname{Ker} d_{1}^{\epsilon}$ is the direct sum $\mathbb{Z} B^{(2)} \oplus I$ where $I=\left\{\sum_{g \notin B^{(2)}} x_{g}[g] \mid x_{g} \in \mathbb{Z}, \sum x_{g}=0\right\}$. Choose some $u \in B \backslash B^{(2)}$. The image of $d_{2}^{\epsilon}$ is spanned by the $\left[g_{1} g_{2}\right]-\epsilon\left(g_{1}\right)\left[g_{2}\right]-\left[g_{1}\right]$. Among them we find

(1) $\left[u^{2}\right]+[u]-[u]=\left[u^{2}\right]$,

(2) $\left[h_{1} h_{2}\right]-\left[h_{1}\right]-\left[h_{2}\right]$ for $h_{1}, h_{2}$ in $B^{(2)}$,

(3) $[u h]-[u]+[h]$ for $h \in B^{(2)}$,

(4) $\left[h^{u}\right]+[h]$ for $h \in B^{(2)}$.

Indeed, the element (4) is the difference of two elements clearly in $\operatorname{Im} d_{2}^{\epsilon},[h u]-[u]-[h]$ and $\left[u h^{u}\right]+\left[h^{u}\right]-[u]$, where $h^{u}=u^{-1} h u$, since $u h^{u}=h u$. By (3), and since $I$ is spanned by the $[h u]-[u]$ for $h \in B^{(2)}$, we see that $H_{1}\left(B, \mathbb{Z}_{\epsilon}\right)$ is generated by the images of the $[h]$ for $h \in B^{(2)}$. It is easy to check that the relations of the form $d_{2}^{\epsilon}\left(\left[g_{1}, g_{2}\right]\right) \equiv 0$ are consequences of (1)-(4), hence $H_{1}\left(B, \mathbb{Z}_{\epsilon}\right)$ is the quotient of $B_{\mathrm{ab}}^{(2)}$ by the relations (1) and (4).

The computation of $B^{(2)}$ can be done for exceptional groups by using the ReidemeisterSchreier method (see [MKS66]) and the presentations of [BMR98] and [BM04]. Note 
that they are known to provide presentations of $B$ for all groups, including the exceptional group $G_{31}$ by [Bes07]. We start from one of these standard presentations of $B$ by braided reflections $\sigma_{1}, \ldots, \sigma_{n}$ and use $\left\{1, \sigma_{1}\right\}$ for Schreier transversal. Then generators for $B^{(2)}$ are given by $\sigma_{1}^{2}, \sigma_{1} \sigma_{2}, \sigma_{1} \sigma_{3}, \ldots, \sigma_{1} \sigma_{n}$ and $\sigma_{2} \sigma_{1}^{-1}, \sigma_{3} \sigma_{1}^{-1}, \ldots, \sigma_{n} \sigma_{1}^{-1}$. We then apply the Reidemeister-Schreier process and find a presentation of $B^{(2)}$ from the relations $R$, $\sigma_{1} R \sigma_{1}^{-1}$ where $R$ runs over the relations for $B$. The presentations obtained for exceptional groups are tabulated in Figure 4 (the column 'ST' refers to the Shephard-Todd number of the group). It is then easy to abelianize these relations. We choose $u=\sigma_{1}$.

In order to get $H_{1}\left(B, \mathbb{Z}_{\epsilon}\right)$ from $B_{\mathrm{ab}}^{(2)}$ we start by adding the relation $\left[\sigma_{1}^{2}\right] \equiv 0$. Note that $\sigma_{1}\left(\sigma_{i} \sigma_{1}^{-1}\right) \sigma_{1}^{-1}=\left(\sigma_{1} \sigma_{i}\right)\left(\sigma_{1}^{-2}\right)$, hence $-\left[\sigma_{i} \sigma_{1}^{-1}\right] \equiv\left[\sigma_{1} \sigma_{i}\right]-\left[\sigma_{1}^{2}\right] \equiv\left[\sigma_{1} \sigma_{i}\right]$, and that $\sigma_{1}\left(\sigma_{1} \sigma_{i}\right) \sigma_{1}^{-1}=\sigma_{1}^{2} \cdot \sigma_{i} \sigma_{1}^{-1}$, hence $-\left[\sigma_{1} \sigma_{i}\right] \equiv\left[\sigma_{i} \sigma_{1}^{-1}\right]$. The relations defining $H_{1}\left(B, \mathbb{Z}_{\epsilon}\right)$ from $B_{\mathrm{ab}}^{(2)}$ thus boil down to $-\left[\sigma_{1} \sigma_{i}\right] \equiv\left[\sigma_{i} \sigma_{1}^{-1}\right]$ and $\left[\sigma_{1}^{2}\right] \equiv 0$.

In order to get $H_{1}\left(B, \mathbb{Z}_{\epsilon}\right)$ for the groups $G(* e, e, r)$, instead of using the complicated presentations of $B$ afforded by [BMR98], we use the semidirect product decomposition described in Section 2. Recall that $B=\mathbb{Z} \ltimes \tilde{A}$ where we denote by $A$ the affine Artin group of type $\tilde{A}_{r-1}$. Then $A$ has Artin generators $\sigma_{1}, \ldots, \sigma_{r}$ and the semidirect product is defined by $\tau \sigma_{i} \tau^{-1}=\sigma_{i+e}$ where addition is considered modulo $r$. From the split exact sequence $1 \rightarrow A \rightarrow B \rightarrow \mathbb{Z} \rightarrow 1$ we get the Hochschild-Serre short exact sequence

$$
\begin{aligned}
0=H_{2}\left(\mathbb{Z}, H_{0}\left(A, \mathbb{Z}_{\epsilon}\right)\right) \rightarrow H_{0}\left(\mathbb{Z}, H_{1}\left(A, \mathbb{Z}_{\epsilon}\right)\right) \rightarrow H_{1}\left(B, \mathbb{Z}_{\epsilon}\right) & \\
& \rightarrow H_{1}\left(\mathbb{Z}, H_{0}\left(A, \mathbb{Z}_{\epsilon}\right)\right) \rightarrow 0
\end{aligned}
$$

with $H_{2}\left(\mathbb{Z}, H_{0}\left(A, \mathbb{Z}_{\epsilon}\right)\right)=0$ since $\mathbb{Z}$ has homological dimension 1 . Since $A$ acts on $\mathbb{Z}_{\epsilon}$ through $\sigma_{i} \mapsto-1$ we have $H_{0}\left(A, \mathbb{Z}_{\epsilon}\right)=\mathbb{Z} / 2 \mathbb{Z}=\mathbb{Z}_{2}$; since $\tau$ acts trivially on $H_{0}\left(A, \mathbb{Z}_{\epsilon}\right)$ we thus get $H_{1}\left(\mathbb{Z}, H_{0}\left(A, \mathbb{Z}_{\epsilon}\right)\right) \simeq H_{1}\left(\mathbb{Z}, \mathbb{Z}_{2}\right) \simeq \mathbb{Z}_{2}$. The short exact sequence thus boils down to $0 \rightarrow H_{0}\left(\mathbb{Z}, H_{1}\left(A, \mathbb{Z}_{\epsilon}\right)\right) \rightarrow H_{1}\left(B, \mathbb{Z}_{\epsilon}\right) \rightarrow \mathbb{Z}_{2} \rightarrow 0$ and our task is reduced to computing $H_{1}\left(A, \mathbb{Z}_{\epsilon}\right)$ while keeping track of the action of $\tau$.

In order to compute $H_{1}\left(A, \mathbb{Z}_{\epsilon}\right)$ we apply the above process. Generators for $A^{(2)}$ are given by $u=\sigma_{1}^{2}, x_{i}=\sigma_{1} \sigma_{i}$ and $y_{i}=\sigma_{i} \sigma_{1}^{-1}$ for $2 \leq i \leq r$, and relations are given by rewriting $R$ and $\sigma_{1} R \sigma_{1}^{-1}$ with $R$ running over the braid relations for $A$. These braid relations are the following (where $|j-i| \geq 2$ actually means that $j, i$ are not connected in the braid diagram):

$$
\begin{aligned}
& 1 \notin\{i, i+1\} \quad \sigma_{i} \sigma_{i+1} \sigma_{i} \sigma_{i+1}^{-1} \sigma_{i}^{-1} \sigma_{i+1}^{-1} \quad \rightsquigarrow y_{i} x_{i+1} y_{i} y_{i+1}^{-1} x_{i}^{-1} y_{i+1}^{-1} \\
& |j-i| \geq 2,1 \notin\{i, j\} \sigma_{i} \sigma_{j} \sigma_{i}^{-1} \sigma_{j}^{-1} \quad \rightsquigarrow y_{i} x_{j} x_{i}^{-1} y_{j}^{-1} \\
& \sigma_{1} \sigma_{2} \sigma_{1} \sigma_{2}^{-1} \sigma_{1}^{-1} \sigma_{2}^{-1} \quad \rightsquigarrow x_{2} y_{2}^{-1} u^{-1} y_{2}^{-1} \\
& i \notin\{2, r\} \quad \sigma_{1} \sigma_{i} \sigma_{1}^{-1} \sigma_{i}^{-1} \quad \rightsquigarrow x_{r} y_{r} u^{-1} y_{i}^{-1} y_{r} \\
& \left(\sigma_{1} R \sigma_{1}^{-1}\right) 1 \notin\{i, i+1\} \quad \sigma_{1} \sigma_{i} \sigma_{i+1} \sigma_{i} \sigma_{i+1}^{-1} \sigma_{i}^{-1} \sigma_{i+1}^{-1} \sigma_{1}^{-1} \rightsquigarrow x_{i} y_{i+1} x_{i} x_{i+1}^{-1} y_{i}^{-1} x_{i+1}^{-1} \\
& |j-i| \geq 2,1 \notin\{i, j\} \sigma_{1} \sigma_{i} \sigma_{j} \sigma_{i}^{-1} \sigma_{j}^{-1} \sigma_{1}^{-1} \quad \rightsquigarrow x_{i} y_{j} y_{i}^{-1} x_{j}^{-1} \\
& \sigma_{1} \sigma_{1} \sigma_{2} \sigma_{1} \sigma_{2}^{-1} \sigma_{1}^{-1} \sigma_{2}^{-1} \sigma_{1}^{-1} \rightsquigarrow u y_{2} u x_{2}^{-1} x_{2}^{-1} \\
& i \notin\{2, r\} \quad \begin{array}{lll}
\sigma_{1} \sigma_{1} \sigma_{i} \sigma_{1}^{-1} \sigma_{i}^{-1} \sigma_{1}^{-1} & \rightsquigarrow u y_{r} u x_{r} x_{i}^{-1}
\end{array}
\end{aligned}
$$


\begin{tabular}{|l|l}
\hline ST & Presentations for the group of even braids
\end{tabular}

$12 v b u=a w v, u a w=v b u a, v b u=b u a w, u a w=w v b$

13 buaw $=a w v, w v b=v b u a, v b u a=b u a w, u a w v=w v b u$

$22 v b u a=a w v b, u a w v=v b u a w, v b u a=b u a w, u a w v=w v b u$

23 aиаиа $=v v, v v v=u a u a u, b u=w, w=u b, b v b=a w a, w a w=v b v$

$24 v v=a u a u, u a u a=v v, a w a w=b v b v, v b v b=w a w a, w=b u b, u b u=w w$,

$a u a w v b=v b u a w, v v b u a w=u a w v b u$

$27 w=b u b, u b u=w w, v v=a u a u, u a u a=v v, a w a w a=b v b v b, v b v b v=w a w a w$, bvwaua $=$ waubv, waubvv $=u b v w a u$

$28 a u a=v, v v=u a u, b u=w, w=u b, b v b v=a w a w, w a w a=v b v b, c u=x, x=u c, c v=a x$, $c v=a x, x a=v c, c w c=b x b, x b x=w c w$

$29 v=a u a, u a u=v v, a x a=c v c, v c v=x a x, b x b=c w c, w c w=x b x, a w a w=b v b v$, $v b v b=w a w a, w=b u, u b=w, x=c u, u c=x, c w a x b v=b v c w a x, x b v c w a=w a x b v c$

30 аиаиа $=v v, v v v=u a u a u, b u=w, w=u b, b v b=a w a, w a w=v b v, c u=x, x=u c$, $c v=a x, x a=v c, c w c=b x b, x b x=w c w$

$31 x=c u c, u c u=x x, a x a=c v c, v c v=x a x, d w d=b y b, y b y=w d w, a y a=d v d, v d v=y a y$, $v b=a w, u a w=v b u, a w=b u a, v b u=w v, y=d u, u d=y, b x=c w, w c=x b, d x=c y$, $y c=x d$

$33 v=a u a, u a u=v v, b v b=a w a, w a w=v b v, c v c=a x a, x a x=v c v, c w c=b x b, x b x=w c w$, $c y c=d x d, x d x=y c y, w=b u, u b=w, x=c u, u c=x, y=d u, u d=y, a y=d v, v d=y a$, $b y=d w, w d=y b, c v b x a w=b x a w c v, x a w c v b=w c v b x a$

$34 v=a u a, u a u=v v, b v b=a w a, w a w=v b v, c v c=a x a, x a x=v c v, c w c=b x b, x b x=w c w$, $c y c=d x d, x d x=y c y, w=b u, u b=w, x=c u, u c=x, y=d u, u d=y, a y=d v, v d=y a$, $b y=d w, w d=y b, d z d=e y e, y e y=z d z, z=e u, u e=z, a z=e v, v e=z a, b z=e w$, $w e=z b, c z=e x, x e=z c, c v b x a w=b x a w c v, x a w c v b=w c v b x a$

$35 a u=v, v=u a, b u b=w, w w=u b u, b v=a w, w a=v b, c u=x, x=u c, c v c=a x a$, $x a x=v c v, c w c=b x b, x b x=w c w, d u=y, y=u d, d v=a y, y a=v d, d w=b y, y b=w d$, $d x d=c y c, y c y=x d x, e u=z, z=u e, e v=a z, z a=v e, e w=b z, z b=w e$, $e x=c z, z c=x e, e y e=d z d, z d z=y e y$

$36 a u=v, v=u a, b u b=w, w w=u b u, b v=a w, w a=v b, c u=x, x=u c, c v c=a x a$, $x a x=v c v, c w c=b x b, x b x=w c w, d u=y, y=u d, d v=a y, y a=v d, d w=b y$, $y b=w d, d x d=c y c, y c y=x d x, e u=z, z=u e, e v=a z, z a=v e, e w=b z, z b=w e$, $e x=c z, z c=x e, e y e=d z d, z d z=y e y, f u=x_{2}, x_{2}=u f, f v=a x_{2}, x_{2} a=v f$, $f w=b x_{2}, x_{2} b=w f, f x=c x_{2}, x_{2} c=x f, f y=d x_{2}, x_{2} d=y f, f z f=e x_{2} e, x_{2} e x_{2}=z f z$

$37 a u=v, v=u a, b u b=w, w w=u b u, b v=a w, w a=v b, c u=x, x=u c, c v c=a x a$, $x a x=v c v, c w c=b x b, x b x=w c w, d u=y, y=u d, d v=a y, y a=v d, d w=b y$, $y b=w d, d x d=c y c, y c y=x d x, e u=z, z=u e, e v=a z, z a=v e, e w=b z, z b=w e$, $e x=c z, z c=x e, e y e=d z d, z d z=y e y, f u=x_{2}, x_{2}=u f, f v=a x_{2}, x_{2} a=v f$, $f w=b x_{2}, x_{2} b=w f, f x=c x_{2}, x_{2} c=x f, f y=d x_{2}, x_{2} d=y f, f z f=e x_{2} e$, $x_{2} e x_{2}=z f z, g u=y_{2}, y_{2}=u g, g v=a y_{2}, y_{2} a=v g, g w=b y_{2}, y_{2} b=w g, g x=c y_{2}$, $y_{2} c=x g, g y=d y_{2}, y_{2} d=y g, g z=e y_{2}, y_{2} e=z g, g x_{2} g=f y_{2} f, y_{2} f y_{2}=x_{2} g x_{2}$

Fig. 4. Presentations for even braid groups of exceptional types. 
Abelianizing and dividing out by the relations $y_{i}=-x_{i}$ yields an abelian presentation for $H_{1}\left(A, \mathbb{Z}_{\epsilon}\right)$ by generators $u, x_{i}$ for $2 \leq i \leq r$ and relations

$$
\begin{array}{ll}
1 \notin\{i, i+1\} & 3 x_{i+1}=3 x_{i} \\
|j-i| \geq 2,1 \notin\{i, j\} & 2 x_{j}=2 x_{i} \\
& 3 x_{2}=0 \\
& 3 x_{r}=0 \\
i \notin\{2, r\} & 2 x_{i}=0
\end{array}
$$

Thus, for $r=3, H_{1}\left(A, \mathbb{Z}_{\epsilon}\right)=\left\langle x_{2}, x_{3} \mid 3 x_{2}=3 x_{3}=0\right\rangle=\mathbb{Z}_{3} x_{2} \oplus \mathbb{Z}_{3} x_{3} \simeq \mathbb{Z}_{3}^{2}$, while for $r=4$,

$$
H_{1}\left(A, \mathbb{Z}_{\epsilon}\right)=\left\langle x_{2}, x_{3}, x_{4} \mid 3 x_{2}=3 x_{4}=0,2 x_{3}=0,2 x_{2}=2 x_{4}, 3 x_{3}=3 x_{2}=3 x_{4}\right\rangle,
$$

hence $H_{1}\left(A, \mathbb{Z}_{\epsilon}\right)=\left\langle x_{2}, x_{4} \mid 3 x_{2}=3 x_{4}=0, x_{2}=x_{4}\right\rangle=\mathbb{Z}_{3} x_{2} \simeq \mathbb{Z}_{3}$. When $r \geq 5$, $H_{1}\left(A, \mathbb{Z}_{\epsilon}\right)$ is generated by $x_{2}, \ldots, x_{r}$, and we have $3 x_{2}=3 x_{r}=0$. We have $2<3<$ $r-1<r$. Then $2 x_{3}=2 x_{r-1}=0$ but $0=3 x_{2}=3 x_{3}$ and $0=3 x_{r}=3 x_{r-1}$. It follows that $x_{3}=0$ and $x_{r-1}=0$. Since $2 x_{3}=2 x_{r}$ and $2 x_{2}=2 x_{r-1}$ we get $x_{2}=x_{r-1}$ and $x_{3}=x_{r}$, hence $x_{i}=0$ for all $i$ and $H_{1}\left(A, \mathbb{Z}_{\epsilon}\right)=0$.

For $r \in\{3,4\}$ it remains to compute the action of $\tau$ on $H_{1}\left(A, \mathbb{Z}_{\epsilon}\right)$. We have $\tau . \sigma_{i}=$ $\sigma_{i+e}$, hence $\tau$. $\left(\sigma_{1} \sigma_{i}\right)=\sigma_{1+e} \sigma_{i+e}=\sigma_{1+e} \sigma_{1}^{-1} \sigma_{1} \sigma_{i+e}$. For $e \equiv 0 \bmod r$ we have $\tau . x_{i}=x_{i}$ and $H_{0}\left(\mathbb{Z}, H_{1}\left(A, \mathbb{Z}_{\epsilon}\right)\right) \simeq H_{1}\left(A, \mathbb{Z}_{\epsilon}\right)$. For $r=3, e \equiv 1 \bmod 3, \tau \cdot x_{2}=\sigma_{2} \sigma_{3} \equiv y_{2}+x_{3} \equiv$ $-x_{2}+x_{3}$ and $\tau \cdot x_{3}=\sigma_{2} \sigma_{1}=\sigma_{2} \sigma_{1}^{-1} \sigma_{1}^{2} \equiv-x_{2}$. It follows that $H_{0}\left(\mathbb{Z}, H_{1}\left(A, \mathbb{Z}_{\epsilon}\right)\right) \simeq \mathbb{Z}_{3}$. For $r=4, e \equiv 1 \bmod 4, \tau \cdot x_{2}=\sigma_{2} \sigma_{3}=\sigma_{2} \sigma_{1}^{-1} \sigma_{1} \sigma_{3} \equiv y_{2}+x_{3} \equiv-x_{2}+x_{3} \equiv-x_{2}$, hence $H_{0}\left(\mathbb{Z}, H_{1}\left(A, \mathbb{Z}_{\epsilon}\right)\right)=0$. For $r=4, e \equiv 2 \bmod 4, \tau \cdot x_{2}=\sigma_{3} \sigma_{4}=\sigma_{3} \sigma_{1}^{-1} \sigma_{1} \sigma_{4} \equiv x_{2}$, hence $H_{0}\left(\mathbb{Z}, H_{1}\left(A, \mathbb{Z}_{\epsilon}\right)\right)=H_{1}\left(A, \mathbb{Z}_{\epsilon}\right)$. Altogether, this yields

Proposition 7.5. For $B=\mathrm{B}(* e, e, r)$, and $r \geq 3$,

$$
\begin{array}{ll}
H_{1}\left(B, \mathbb{Z}_{\epsilon}\right) \simeq \mathbb{Z}_{2} & \text { for } r \geq 5, \\
H_{1}\left(B, \mathbb{Z}_{\epsilon}\right) \simeq \mathbb{Z}_{6} & \text { for } r=4, e \equiv 0,2 \bmod 4, \\
H_{1}\left(B, \mathbb{Z}_{\epsilon}\right) \simeq \mathbb{Z}_{2} & \text { for } r=4, e \equiv 1 \bmod 4, \\
H_{1}\left(B, \mathbb{Z}_{\epsilon}\right) \simeq \mathbb{Z}_{3} \oplus \mathbb{Z}_{3} \oplus \mathbb{Z}_{2} & \text { for } r=3, e \equiv 0 \bmod 4, \\
H_{1}\left(B, \mathbb{Z}_{\epsilon}\right) \simeq \mathbb{Z}_{6} & \text { for } r=3, e \equiv 1 \bmod 4 .
\end{array}
$$

Finally, for groups of type $G(e, e, r)$, we use the Dehornoy-Lafont complex associated to the Corran-Picantin monoid. The 1-cells $[s]$ are mapped to $(\epsilon(s)-1)[\emptyset]=-2[\emptyset]$, hence the 1-cycles are spanned by the $[s]-[t]$ for $s, t$ two atoms. We have $d_{\epsilon}\left[s_{j}, s_{i}\right]=$ $2\left(s_{j}-s_{i}\right)$ when $|j-i| \geq 2, d_{\epsilon}\left[t_{0}, t_{i}\right]=-t_{i}+t_{i+1}+t_{0}-t_{1}, d_{\epsilon}\left[s_{3}, t_{i}\right]=3 t_{i}-3 s_{3}$, $d_{\epsilon}\left[s_{i}, t_{0}\right]=2\left(s_{i}-t_{0}\right)$ for $i \geq 4$, and $d_{\epsilon}\left[s_{i+1}, s_{i}\right]=3\left(s_{i+1}-s_{i}\right)$. Since a basis of the 1 -cycles is given by the $t_{i}-t_{0}, t_{0}-s_{3}, s_{3}-s_{4}, \ldots, s_{r-1}-s_{r}, H_{1}\left(B, \mathbb{Z}_{\epsilon}\right)$ is spanned by $t_{1}-t_{0}, t_{0}-s_{3}, \ldots, s_{r-1}-s_{r}$, each of these elements being annihilated by 3 . From $d_{\epsilon}\left[s_{i}, t_{0}\right]=2\left(s_{i}-t_{0}\right)$ for $i \geq 4$ we get $s_{4}-s_{3} \equiv s_{3}-t_{0}$, from $d_{\epsilon}\left[s_{5}, s_{3}\right]=2\left(s_{5}-s_{3}\right)$ we get $s_{5}-s_{4} \equiv s_{4}-s_{3}$, and so on. Finally, from

$d_{\epsilon}\left[t_{1}, s_{4}\right]=2\left(t_{1}-s_{4}\right)=2\left(t_{1}-t_{0}\right)+2\left(t_{0}-s_{3}\right)+2\left(s_{3}-s_{4}\right) \equiv 2\left(t-1-t_{0}\right)+\left(t_{0}-s_{3}\right)$ 
Table 11. Homology of exceptional groups.

\begin{tabular}{l|ccccccc} 
& $H_{0}$ & $H_{1}$ & $H_{2}$ & $H_{3}$ & $H_{4}$ & $H_{5}$ & $H_{6}$ \\
\hline$G_{12}$ & $\mathbb{Z}$ & $\mathbb{Z}$ & 0 & & & & \\
$G_{13}$ & $\mathbb{Z}$ & $\mathbb{Z}^{2}$ & $\mathbb{Z}$ & & & & \\
$G_{22}$ & $\mathbb{Z}$ & $\mathbb{Z}$ & 0 & & & & \\
$G_{24}$ & $\mathbb{Z}$ & $\mathbb{Z}$ & $\mathbb{Z}$ & $\mathbb{Z}$ & & & \\
$G_{27}$ & $\mathbb{Z}$ & $\mathbb{Z}$ & $\mathbb{Z}_{3} \times \mathbb{Z}$ & $\mathbb{Z}$ & & & \\
$G_{29}$ & $\mathbb{Z}$ & $\mathbb{Z}$ & $\mathbb{Z}_{2} \times \mathbb{Z}_{4}$ & $\mathbb{Z}_{2} \times \mathbb{Z}$ & $\mathbb{Z}$ & & \\
$G_{31}$ & $\mathbb{Z}$ & $\mathbb{Z}$ & $\mathbb{Z}_{6}$ & $\mathbb{Z}$ & $\mathbb{Z}$ & & \\
$G_{33}$ & $\mathbb{Z}$ & $\mathbb{Z}$ & $\mathbb{Z}_{6}$ & $\mathbb{Z}_{6}$ & $\mathbb{Z}$ & $\mathbb{Z}$ & \\
$G_{34}$ & $\mathbb{Z}$ & $\mathbb{Z}$ & $\mathbb{Z}_{6}$ & $\mathbb{Z}_{6}$ & $?$ & $?$ & $?$
\end{tabular}

Table 12. Homology of exceptional Artin groups (after Salvetti).

\begin{tabular}{l|ccccccccc} 
& $H_{0}$ & $H_{1}$ & $H_{2}$ & $H_{3}$ & $H_{4}$ & $H_{5}$ & $H_{6}$ & $H_{7}$ & $H_{8}$ \\
\hline$I_{2}(2 m)$ & $\mathbb{Z}$ & $\mathbb{Z}^{2}$ & $\mathbb{Z}$ & & & & & & \\
$I_{2}(2 m+1)$ & $\mathbb{Z}$ & $\mathbb{Z}$ & 0 & & & & & & \\
$H_{3}=G_{23}$ & $\mathbb{Z}$ & $\mathbb{Z}$ & $\mathbb{Z}$ & $\mathbb{Z}$ & & & & & \\
$H_{4}=G_{30}$ & $\mathbb{Z}$ & $\mathbb{Z}$ & $\mathbb{Z}_{2}$ & $\mathbb{Z}$ & $\mathbb{Z}$ & & & & \\
$F_{4}=G_{28}$ & $\mathbb{Z}$ & $\mathbb{Z}^{2}$ & $\mathbb{Z}^{2}$ & $\mathbb{Z}^{2}$ & $\mathbb{Z}$ & & & & \\
$E_{6}=G_{35}$ & $\mathbb{Z}$ & $\mathbb{Z}$ & $\mathbb{Z}_{2}$ & $\mathbb{Z}_{2}$ & $\mathbb{Z}_{6}$ & $\mathbb{Z}_{3}$ & 0 & & \\
$E_{7}=G_{36}$ & $\mathbb{Z}$ & $\mathbb{Z}$ & $\mathbb{Z}_{2}$ & $\mathbb{Z}_{2}^{2}$ & $\mathbb{Z}_{6}^{2}$ & $\mathbb{Z}_{3} \times \mathbb{Z}_{6}$ & $\mathbb{Z}$ & $\mathbb{Z}$ & \\
$E_{8}=G_{37}$ & $\mathbb{Z}$ & $\mathbb{Z}$ & $\mathbb{Z}_{2}$ & $\mathbb{Z}_{2}$ & $\mathbb{Z}_{2} \times \mathbb{Z}_{6}$ & $\mathbb{Z}_{3} \times \mathbb{Z}_{6}$ & $\mathbb{Z}_{2} \times \mathbb{Z}_{6}$ & $\mathbb{Z}$ & $\mathbb{Z}$
\end{tabular}

Table 13. Abelianization of even braids and $H_{1}\left(B, \mathbb{Z}_{\epsilon}\right)$.

\begin{tabular}{|c|c|c|}
\hline $\mathrm{ST}$ & $B_{\mathrm{ab}}^{(2)}$ & $H_{1}\left(B, \mathbb{Z}_{\epsilon}\right)$ \\
\hline 12 & $\mathbb{Z}_{3} \times \mathbb{Z}$ & $\mathbb{Z}_{3}$ \\
13 & $\mathbb{Z} \times \mathbb{Z}$ & $\mathbb{Z}_{2}$ \\
22 & $\mathbb{Z}$ & 0 \\
23 & $\mathbb{Z}$ & 0 \\
24 & $\mathbb{Z}$ & 0 \\
27 & $\mathbb{Z}$ & 0 \\
28 & $\mathbb{Z} \times \mathbb{Z}$ & $\mathbb{Z}_{2}$ \\
29 & $\mathbb{Z}$ & 0 \\
30 & $\mathbb{Z}$ & 0 \\
31 & $\mathbb{Z}$ & 0 \\
33 & $\mathbb{Z}$ & 0 \\
34 & $\mathbb{Z}$ & 0 \\
35 & $\mathbb{Z}$ & 0 \\
36 & $\mathbb{Z}$ & 0 \\
37 & $\mathbb{Z}$ & 0 \\
\hline
\end{tabular}


we get $t_{1}-t_{0} \equiv t_{0}-s_{3}$. It follows that, for $r \geq 4, H_{1}\left(B, \mathbb{Z}_{\epsilon}\right)$ is generated by $t_{1}-t_{0}$, hence $H_{1}\left(B, \mathbb{Z}_{\epsilon}\right) \simeq \mathbb{Z}_{3}$; for $r=3$, it is generated by $t_{1}-t_{0}$ and $t_{0}-s_{3}$ and $H_{1}\left(B, \mathbb{Z}_{\epsilon}\right) \simeq \mathbb{Z}_{3} \oplus \mathbb{Z}_{3}$; and it is generated by $t_{1}-t_{0}$ for $r=2$.

The case $e=1$ (that is, of the usual braid group) follows the same pattern. On the whole, we get the following.

Proposition 7.6. For the groups $\mathrm{B}(e, e, r)$ with $e \geq 2, H_{1}\left(B, \mathbb{Z}_{\epsilon}\right) \simeq \mathbb{Z}_{3}$ if $r \geq 4$. If $r=3$ then $H_{1}\left(B, \mathbb{Z}_{\epsilon}\right) \simeq \mathbb{Z}_{3} \oplus \mathbb{Z}_{3}$. If $r=2$ then $H_{1}\left(B, \mathbb{Z}_{\epsilon}\right) \simeq \mathbb{Z}$. When $e=1$, we have $H_{1}\left(B, \mathbb{Z}_{\epsilon}\right)=0$ for $r=2$ or $r \geq 5$, and $H_{1}\left(B, \mathbb{Z}_{\epsilon}\right)=\mathbb{Z}_{3}$ if $r=3$ or $r=4$.

Acknowledgments. The first computations for the exceptional groups were made with the help of Jean Michel. We are grateful to Y. Lafont and G. Lehrer for discussions, and to the referee for his suggestions. The second author benefited from the ANR grant ANR-09-JCJC-0102-01.

\section{References}

[Ban76] Bannai, E.: Fundamental groups of the spaces of regular orbits of the finite unitary reflection groups of dimension 2. J. Math. Soc. Japan 28, 447-454 (1976) Zbl 0326.57015 MR 0407199

[BC06] Bessis, D., Corran, R.: Non-crossing partitions of type $(e, e, r)$. Adv. Math. 202, 1-49 (2006) Zbl 1128.20024 MR 2218819

[Bes07] Bessis, D.: Finite complex reflection arrangements are $K(\pi, 1)$. arXiv:math/0610777v3[math:GT] (2007)

[BM04] Bessis, D., Michel, J.: Explicit presentations for exceptional braid groups. Experiment. Math. 13, 257-266 (2004) Zbl 1092.20033 MR 2103323

[BMR98] Broué, M., Malle, G., Rouquier, R.: Complex reflection groups, braid groups, Hecke algebras. J. Reine Angew. Math. 500, 127-190 (1998) Zbl 0921.20046

[Bon02] Bonnafé, C.: Une (nouvelle?) construction du groupe de réflexion complexe $G_{31}$. Bull. Math. Soc. Sci. Math. Roumanie (N.S.) 45 (93), 133-143 (2002) Zbl 1084.20509 MR 2098685

[Bri73] Brieskorn, E.: Sur les groupes de tresses [d'après V. I. Arnol'd]. In: Séminaire Bourbaki, 24ème année (1971/1972), exp. 401, Lecture Notes in Math. 317, Springer, Berlin, 21-44 (1973) Zbl 0277.55003 MR 0422674

[Bro82] Brown, K. S.: Cohomology of Groups. Grad. Texts in Math. 87, Springer, New York (1982) Zbl 0584.20036 MR 0672956

[Ca106] Callegaro, F.: The homology of the Milnor fiber for classical braid groups. Algebr. Geom. Topol. 6, 1903-1923 (2006) Zbl 1166.20044 MR 2263054

[CMS08] Callegaro, F., Moroni, D., Salvetti, M.: Cohomology of affine Artin groups and applications. Trans. Amer. Math. Soc. 360, 4169-4188 (2008) Zbl 1191.20056 MR 2395168

[CMW04] Charney, R., Meier, J., Whittlesey, K.: Bestvina's normal form complex and the homology of Garside groups. Geom. Dedicata 105, 171-188 (2004) Zbl 1064.20044 MR 2057250

[Coh76] Cohen, F. R.: The homology of $\mathcal{C}_{n+1}$-spaces, $n \geq 0$. In: Homology of Iterated Loop Spaces, Lecture Notes in Math. 533, Springer, 207-353 (1976) Zbl 0334.55009 MR 0436146

[Coh88] Cohen, F. R.: Artin's braid groups, classical homotopy theory, and sundry other curiosities. In: Braids (Santa Cruz, CA, 1986), Contemp. Math. 78, Amer. Math. Soc., Providence, RI, 167-206 (1988) Zbl 0577.20026 MR 0975079 
[CP] Corran, R., Picantin, M.: A new Garside structure for the braid groups of type $(e, e, r)$. J. London Math. Soc. 84, 689-711 (2011) Zbl 1239.20042 MR 2855797

[Cri99] Crisp, J.: Injective maps between Artin groups. In: Geometric Group Theory Down Under (Canberra, 1996), de Gruyter, Berlin, 119-137 (1999) Zbl 1001.20034 MR 1714842

[Del72] Deligne, P.: Les immeubles des groupes de tresses généralisés. Invent. Math. 17, 273 302 (1972) Zbl 0238.20034 MR 0422673

[DL03] Dehornoy, P., Lafont, Y.: Homology of Gaussian groups. Ann. Inst. Fourier (Grenoble) 53, 489-540 (2003) Zbl 1100.20036 MR 1990005

[DMM11] Digne, F., Marin, I., Michel, J.: The center of pure complex braid groups. J. Algebra 347, 206-213 (2011) Zbl 1241.20039 MR 2846406

[DP99] Dehornoy, P., Paris, L.: Gaussian groups and Garside groups, two generalisations of Artin groups. Proc. London Math. Soc. (3) 79, 569-604 (1999) Zbl 1030.20021 MR 1710165

[DPS01] De Concini, C., Procesi, C., Salvetti, M.: Arithmetic properties of the cohomology of braid groups. Topology 40, 739-751 (2001) Zbl 0999.20046 MR 1851561

[DPSS99] De Concini, C., Procesi, C., Salvetti, M., Stumbo, F.: Arithmetic properties of the cohomology of Artin groups. Ann. Scuola Norm. Sup. Pisa Cl. Sci. (4) 28, 695-717 (1999) Zbl 0973.20025 MR 1760537

[FN62] Fadell, E., Neuwirth, L.: Configuration spaces. Math. Scand. 10, 111-118 (1962) Zbl 0136.44104 MR 0141126

[Fuk70] Fuks, D. B.: Cohomology of the braid group mod 2. Funct. Anal. Appl. 4, 143-151 (1970) Zbl 0222.57031 MR 0274463

[Fuk74] Fuks, D. B.: Quillenization and bordism. Funktsional. Anal. i Prilozhen. 8, no. 1, 36-42 (1974) (in Russian) Zbl 0324.57024 MR 0343301

[Gor81] Gorjunov, V. V.: Cohomology of braid groups of series $C$ and $D$. Trudy Moskov. Mat. Obshch. 42, 234-242 (1981) (in Russian) Zbl 0547.55016 MR 0622003

[IP02] Kent IV, R. P., Peifer, D.: A geometric and algebraic description of annular braid groups. Internat. J. Algebra Comput. 12, 85-97 (2002) MR 1902362

[Kob90] Kobayashi, Y.: Complete rewriting systems and homology of monoid algebras. J. Pure Appl. Algebra 65, 263-275 (1990) Zbl 0711.20035 MR 1072284

[Leh95] Lehrer, G. I.: Poincaré polynomials for unitary reflection groups. Invent. Math. 120, 411-425 (1995) Zbl 0831.20049 MR 1334478

[Leh04] Lehrer, G. I.: Rational points and cohomology of discriminant varieties. Adv. Math. 186, 229-250 (2004) Zbl 1077.14025 MR 2065513

[MKS66] Magnus, W., Karrass, A., Solitar, D.: Combinatorial Group Theory: Presentations of Groups in Terms of Generators and Relations. Interscience Publ., New York (1966) Zbl 0138.25604 MR 0207802

[Mic] Michel, J.: Homepage of the development version of the GAP part of CHEVIE. http://www.math.jussieu.fr/ jmichel/chevie/chevie.html.

[Nak83] Nakamura, T.: A note on the $K(\pi, 1)$ property of the orbit space of the unitary reflection group $G(m, l, n)$. Sci. Papers College Arts Sci. Univ. Tokyo 33, 1-6 (1983) Zbl 0524.20027 MR 0714667

[OT92] Orlik, P., Terao, H.: Arrangements of Hyperplanes. Grundlehren Math. Wiss. 300, Springer, Berlin (1992) Zbl 0757.55001 MR 1217488

[Par04] Paris, L.: Artin groups of spherical type up to isomorphism. J. Algebra 281, 666-678 (2004) Zbl 1080.20033 MR 2098788

[Pic00] Picantin, M.: Petits groupes gaussiens. Ph.D. thesis, Univ. de Caen (2000) 
[Rea76] Read, E. W.: On the Schur multipliers of the finite imprimitive unitary reflection groups $G(m, p, n)$. J. London Math. Soc. (2) 13, 150-154 (1976) Zbl 0328.20011 MR 0399243

[Sal94] Salvetti, M.: The homotopy type of Artin groups. Math. Res. Lett. 1, 565-577 (1994) Zbl 0847.55011 MR 1386847

[Seg73] Segal, G.: Configuration-spaces and iterated loop-spaces. Invent. Math. 21, 213-221 (1973) Zbl 0267.55020 MR 0331377

[ST54] Shephard, G. C., A. Todd, J.: Finite unitary reflection groups. Canad. J. Math. 6, 274304 (1954) Zbl 0055.14305 MR 0059914

[Vaĭ78] Vaŭnštel̆n, F. V.: The cohomology of braid groups. Funktsional. Anal. i Prilozhen. 12, no. 2, 72-73 (1978) (in Russian) MR 0498903

[vdH77] van der Hout, R.: The Schur multipliers of the finite primitive complex reflection groups. Nederl. Akad. Wetensch. Proc. Ser. A 80 = Indag. Math. 39, 101-113 (1977) Zbl 0361.20014 MR 0442105 